\title{
Topological Hochschild homology of Thom spectra and the free loop space
}

\author{
ANDREW J BLUMBERG \\ RALPH L COHEN \\ CHRISTIAN SCHLICHTKRULL
}

\begin{abstract}
We describe the topological Hochschild homology of ring spectra that arise as Thom spectra for loop maps $f: X \rightarrow B F$, where $B F$ denotes the classifying space for stable spherical fibrations. To do this, we consider symmetric monoidal models of the category of spaces over $B F$ and corresponding strong symmetric monoidal Thom spectrum functors. Our main result identifies the topological Hochschild homology as the Thom spectrum of a certain stable bundle over the free loop space $L(B X)$. This leads to explicit calculations of the topological Hochschild homology for a large class of ring spectra, including all of the classical cobordism spectra $M O, M S O$, $M U$, etc, and the Eilenberg-Mac Lane spectra $H \mathbb{Z} / p$ and $H \mathbb{Z}$.
\end{abstract}

19D55, 55N20; 18G55, 55P43, 55P47, 55R25

\section{Introduction}

Many interesting ring spectra arise naturally as Thom spectra. It is well-known that one may associate a Thom spectrum $T(f)$ to any map $f: X \rightarrow B F$, where $B F$ denotes the classifying space for stable spherical fibrations. This construction is homotopy invariant in the sense that the stable homotopy type of $T(f)$ only depends on the homotopy class of $f$; see Lewis, May, Steinberger and McClure [23] and Mahowald [27]. Furthermore, if $f$ is a loop map, then it follows from a result of Lewis [23] that $T(f)$ inherits the structure of an $A_{\infty}$ ring spectrum. In this case the topological Hochschild homology spectrum $\operatorname{THH}(T(f))$ is defined. For example, all of the Thom spectra $M G$ representing the classical cobordism theories (where $G$ denotes one of the stabilized Lie groups $O$, SO, Spin, $U$ or $\mathrm{Sp}$ ) arise from canonical infinite loop maps $B G \rightarrow B F$. In this paper we provide an explicit description of the topological Hochschild homology of such a ring spectrum $T(f)$ in terms of the Thom spectrum of a certain stable bundle over the free loop space. In order to state our main 
result we begin by recalling some elementary results about the free loop space $L(B)$ of a connected space $B$. Fixing a base point in $B$, we have the usual fibration sequence

$$
\Omega(B) \longrightarrow L(B) \longrightarrow B
$$

obtained by evaluating a loop at the base point of $S^{1}$. This sequence is split by the inclusion $B \rightarrow L(B)$ as the constant loops. When $B$ has the structure of a homotopy associative and commutative $\mathrm{H}-$-space, $L(B)$ also has such a structure and the composition

$$
\Omega(B) \times B \longrightarrow L(B) \times L(B) \longrightarrow L(B)
$$

is an equivalence of $\mathrm{H}-$ spaces. If we assume that $B$ has the homotopy type of a $\mathrm{CW}-$ complex, then the same holds for $L(B)$ and inverting the above equivalence specifies a well-defined homotopy class $L(B) \stackrel{\simeq}{\rightarrow} \Omega(B) \times B$. Applying this to the delooping $B^{2} F$ of the infinite loop space $B F$, we obtain a splitting

$$
L\left(B^{2} F\right) \simeq \Omega\left(B^{2} F\right) \times B^{2} F \simeq B F \times B^{2} F .
$$

Let $\eta: S^{3} \rightarrow S^{2}$ denote the unstable Hopf map and also in mild abuse of notation the map obtained by precomposing as follows:

$$
\eta: B^{2} F \simeq \operatorname{Map}_{*}\left(S^{2}, B^{4} F\right) \stackrel{\eta^{*}}{\longrightarrow} \operatorname{Map}_{*}\left(S^{3}, B^{4} F\right) \simeq B F .
$$

The following result is the main theorem of the paper.

Theorem 1 Let $f: X \rightarrow B F$ be the loop map associated to a map of connected based spaces $B f: B X \rightarrow B^{2} F$. Then there is a natural stable equivalence

$$
\operatorname{THH}(T(f)) \simeq T\left(L^{\eta}(B f)\right),
$$

where $L^{\eta}(B f)$ denotes the composite

$$
L^{\eta}(B f): L(B X) \stackrel{L(B f)}{\longrightarrow} L\left(B^{2} F\right) \simeq B F \times B^{2} F \stackrel{\text { id } \times \eta}{\longrightarrow} B F \times B F \longrightarrow B F .
$$

Here the last arrow represents multiplication in the $H$-space $B F$.

When $f$ is the constant map, the Thom spectrum $T(f)$ is equivalent to the spherical group ring $\Sigma^{\infty} \Omega(B X)_{+}$, where + indicates a disjoint base point. In this case we recover the stable equivalence of Bökstedt and Waldhausen,

$$
\operatorname{THH}\left(\Sigma^{\infty} \Omega(B X)_{+}\right) \simeq \Sigma^{\infty} L(B X)_{+} .
$$

The real force of Theorem 1 comes from the fact that the Thom spectrum $T\left(L^{\eta} B f\right)$ can be analyzed effectively in many cases. We will say that $f$ is an $n$-fold loop map if 
we have an $(n-1)$-connected based space $B^{n} X$ and a homotopy commutative diagram of the form

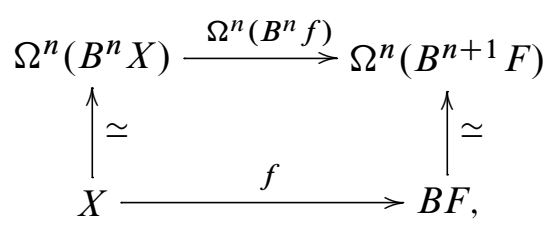

where the vertical maps are equivalences as indicated. When $X$ is a 2 -fold loop space, the product decomposition in (1-1) can be applied to $L(B X)$. We shall then prove the following.

Theorem 2 If $f$ is a 2 -fold loop map, then there is a stable equivalence

$$
\operatorname{THH}(T(f)) \simeq T(f) \wedge T(\eta \circ B f),
$$

where $T(\eta \circ B f)$ denotes the Thom spectrum of $B X \stackrel{B f}{\rightarrow} B^{2} F \stackrel{\eta}{\rightarrow} B F$.

For 3-fold loop maps we can describe $\operatorname{THH}(T(f))$ without reference to $\eta$.

Theorem 3 If $f$ is a 3-fold loop map, then there is a stable equivalence

$$
\operatorname{THH}(T(f)) \simeq T(f) \wedge B X_{+} .
$$

If $f$ is an infinite loop map one can realize the stable equivalence in Theorem 3 as an equivalence of $E_{\infty}$ ring spectra. This is carried out by the first author [5] and the third author [39], working respectively with the $S$-module approach of Elmendorf, Kriz, Mandell and May [16] and the symmetric spectrum approach of Hovey, Shipley and Smith [20] and Mandell, May, Schwede and Shipley [31] to structured ring spectra. The operadically sophisticated reader will note that the term " $n$-fold loop maps" is a deviceindependent way of describing maps between group-complete spaces that are structured by $E_{n}$ operads (that is, operads equivalent to the little $n$-cubes operad). We have chosen this elementary description since this is the kind of input data one encounters most often in the applications and since many of the examples in the literature (such as the ones in Mahowald [27]) are formulated in this language. For the technical part of our work it will be important to pass back and forth between loop maps and maps structured by operads and we explain how to do this in Appendix A.

It is known by Brun, Fiedorowicz and Vogt [11] and Mandell [30] that if $T$ is an $E_{n}$ ring spectrum, then $\operatorname{THH}(T)$ is an $E_{n-1}$ ring spectrum. We expect the following strengthening of Theorem 3 to hold: if $X$ is a grouplike $E_{n}$ space and $f: X \rightarrow B F$ an $E_{n}$ map, then the equivalence in Theorem 3 is an equivalence of $E_{n-2}$ ring spectra. 
Despite the fact that the spectra on both sides of the equivalence are $E_{n-1}$ ring spectra, the splitting of Equation (1-1) is only an equivalence of $E_{n-2}$ spaces and so we cannot hope for an equivalence of $E_{n-1}$ ring spectra.

Finally, note that the equivalences we establish in the preceding theorems are nonequivariant equivalences; ie, we ignore the circle action on the topological Hochschild homology spectrum arising from the cyclic structure. In particular, the equivalences in Theorem 2 and Theorem 3 are nonequivariant since they are derived from the nonequivariant decomposition of the free loop space in (1-1). Although we expect an equivariant analogue of Theorem 1, further work on the theory of equivariant Thom spectra is required to make precise statements possible.

\section{The classical cobordism spectra}

Let $G$ be one of the stabilized Lie groups $O$, SO, Spin, $U$ or $\mathrm{Sp}$. Then the Thom spectrum $M G$ arises from an infinite loop map $B G \rightarrow B F$ and so Theorem 3 applies to give a stable equivalence:

$$
\operatorname{THH}(M G) \simeq M G \wedge B B G_{+} .
$$

Spelling this out using the Bott periodicity theorem, we get the following corollary.

Corollary 1.1 There are stable equivalences of spectra

$$
\begin{aligned}
& \operatorname{THH}(M O) \simeq M O \wedge(U / O)\langle 1\rangle_{+} \\
& \operatorname{THH}(M S O) \simeq M S O \wedge(U / O)\langle 2\rangle_{+} \\
& \operatorname{THH}(M S p i n) \simeq M \operatorname{Spin} \wedge(U / O)\langle 3\rangle_{+} \\
& \operatorname{THH}(M U) \simeq M U \wedge S U_{+} \\
& \operatorname{THH}(M S p) \simeq M \operatorname{Sp} \wedge(U / \mathrm{Sp})\langle 1\rangle_{+},
\end{aligned}
$$

where here $(U / O)\langle n\rangle$ and $(U / \mathrm{Sp})\langle n\rangle$ denote the $n$-connected covers of $U / O$ and $U / \mathrm{Sp}$ respectively.

These results also admit a cobordism interpretation.

Corollary 1.2 Let $G$ be one of the stabilized Lie groups considered above, and let $\Omega_{*}^{G}$ denote the corresponding $G$-bordism theory. Then there is an isomorphism

$$
\pi_{*} \operatorname{THH}(M G) \simeq \Omega_{*}^{G}(B B G) .
$$


There are many other examples of cobordism spectra for which Theorem 3 applies; see Stong [47]. In the case of the identity map $B F \rightarrow B F$ we get the spectrum $M F$, and we again have a stable equivalence

$$
\mathrm{THH}(M F) \simeq M F \wedge B B F_{+} .
$$

\section{The Eilenberg-Mac Lane spectra $H \mathbb{Z} / p$ and $H \mathbb{Z}$}

Another application of our results is to the calculation of the topological Hochschild homology of the Eilenberg-Mac Lane spectra $H \mathbb{Z} / p$ and $H \mathbb{Z}$. These calculations are originally due to Bökstedt [8] using a very different approach. Our starting point here is the fact that these spectra can be realized as Thom spectra. For $H \mathbb{Z} / 2$ this is a theorem of Mahowald [27]; if $f: \Omega^{2}\left(S^{3}\right) \rightarrow B F$ is an extension of the generator of $\pi_{1} B F$ to a 2 -fold loop map, then $T(f)$ is equivalent to $H \mathbb{Z} / 2$; see also Cohen, May and Taylor [14]. In general, given a connected space $X$ and a map $f: X \rightarrow B F$, the associated Thom spectrum has $\pi_{0} T(f)$ equal to $\mathbb{Z}$ or $\mathbb{Z} / 2$, depending on whether $T(f)$ is oriented or not. Hence $H \mathbb{Z} / p$ cannot be realized as a Thom spectrum for a map to $B F$ when $p$ is odd. However, by an observation due to Hopkins [28], if one instead considers the classifying space $B F_{(p)}$ for $p$-local spherical fibrations, then $H \mathbb{Z} / p$ may be realized as the $p$-local Thom spectrum associated to a certain 2-fold loop map $f: \Omega^{2}\left(S^{3}\right) \rightarrow B F_{(p)}$. We recall the definition of $B F_{(p)}$ and the $p$-local approach to Thom spectra in Section 3.4. Our methods work equally well in the $p$-local setting and we shall prove that the $p$-local version of Theorem 2 applies to give the following result.

Theorem 1.3 There is a stable equivalence

$$
\operatorname{THH}(H \mathbb{Z} / p) \simeq H \mathbb{Z} / p \wedge \Omega\left(S^{3}\right)_{+}
$$

for each prime $p$.

On the level of homotopy groups this implies that

$$
\pi_{*} \operatorname{THH}(H \mathbb{Z} / p)=H_{*}\left(\Omega\left(S^{3}\right), \mathbb{Z} / p\right)=\mathbb{Z} / p[x],
$$

where $x$ has degree 2; see eg, Whitehead [51, Theorem 1.18]. In the case of the Eilenberg-Mac Lane spectrum $H \mathbb{Z}_{(p)}$ for the $p$-local integers we instead consider the 2-fold loop space $\Omega^{2}\left(S^{3}\langle 3\rangle\right)$ where $S^{3}\langle 3\rangle$ is the 3-connected cover of $S^{3}$. Arguing as in [14] it follows that the $p$-local Thom spectrum of the 2 -fold loop map

$$
\Omega^{2}\left(S^{3}\langle 3\rangle\right) \longrightarrow \Omega^{2}\left(S^{3}\right) \longrightarrow B F_{(p)}
$$


is equivalent to $H \mathbb{Z}_{(p)}$. Using this we show that the $p$-local version of Theorem 2 applies to give a stable equivalence

$$
\operatorname{THH}\left(H \mathbb{Z}_{(p)}\right) \simeq H \mathbb{Z}_{(p)} \wedge \Omega\left(S^{3}\langle 3\rangle\right)_{+}
$$

for each prime $p$. Since topological Hochschild homology commutes with localization this has the following consequence for the integral Eilenberg-Mac Lane spectrum.

Theorem 1.4 There is a stable equivalence

$$
\mathrm{THH}(H \mathbb{Z}) \simeq H \mathbb{Z} \wedge \Omega\left(S^{3}\langle 3\rangle\right)_{+}
$$

where $S^{3}\langle 3\rangle$ denotes the 3-connected cover of $S^{3}$.

This gives that

$$
\pi_{i} \operatorname{THH}(H \mathbb{Z})=H_{i}\left(\Omega\left(S^{3}\langle 3\rangle\right), \mathbb{Z}\right)= \begin{cases}\mathbb{Z} & \text { for } i=0 \\ \mathbb{Z} /\left(\frac{i+1}{2}\right) & \text { for } i>0 \text { odd } \\ 0 & \text { for } i>0 \text { even }\end{cases}
$$

The last isomorphism is easily obtained by applying the Serre spectral sequence to the fibration sequence

$$
S^{1} \longrightarrow \Omega\left(S^{3}\langle 3\rangle\right) \longrightarrow \Omega\left(S^{3}\right) .
$$

An alternative approach to the calculation of $\operatorname{THH}(H \mathbb{Z})$ has been developed by the first author in [5]. One may also ask if a similar approach can be used for the EilenbergMac Lane spectra $H \mathbb{Z} / p^{n}$ in general. This turns out not to be the case since these spectra cannot be realized as $A_{\infty}$ Thom spectra for $n>1$; see [5] for details.

\section{Thom spectra arising from systems of groups}

A more geometric starting point for the construction of Thom spectra is to consider systems of groups $G_{n}$ equipped with compatible homomorphisms to the general linear groups $\mathrm{GL}_{n}(\mathbb{R})$. We write $M G$ for the associated Thom spectrum whose $n$-th space is the Thom space of the vector bundle represented by $B G_{n} \rightarrow B \mathrm{GL}_{n}(\mathbb{R})$. If the groups $G_{n}$ come equipped with suitable associative pairings $G_{m} \times G_{n} \rightarrow G_{m+n}$, then $M G$ inherits a multiplicative structure. For example, we have the commutative symmetric ring spectra $M \Sigma$ and $M G L(\mathbb{Z})$ associated to the symmetric groups $\Sigma_{n}$ and the general linear groups $\mathrm{GL}_{n}(\mathbb{Z})$. In Section 3.5 we show how to deduce the following results from Theorem 3. 
Theorem 1.5 There is a stable equivalence

$$
\mathrm{THH}(M \Sigma) \simeq M \Sigma \wedge \widetilde{Q}\left(S^{1}\right)_{+}
$$

where $\widetilde{Q}\left(S^{1}\right)$ denotes the homotopy fiber of the canonical map $Q\left(S^{1}\right) \rightarrow S^{1}$.

Here $Q\left(S^{1}\right)$ denotes the infinite loop space associated to the suspension spectrum $\Sigma^{\infty}\left(S^{1}\right)$.

Theorem 1.6 There is a stable equivalence

$$
\operatorname{THH}(M \mathrm{GL}(\mathbb{Z})) \simeq M \mathrm{GL}(\mathbb{Z}) \wedge B\left(B \mathrm{GL}(\mathbb{Z})^{+}\right)_{+}
$$

where $B\left(B \mathrm{GL}(\mathbb{Z})^{+}\right)$denotes the first space in the 0 -connected cover of the algebraic $K$-theory spectrum for $\mathbb{Z}$.

There is a plethora of examples of this kind, involving for instance braid groups, automorphism groups of free groups and general linear groups.

We finally mention an application of our results in connection with the analysis of quasisymmetric functions. Let $\mathbb{C} P^{\infty} \rightarrow B U$ be the canonical map obtained by identifying $\mathbb{C} P^{\infty}$ with $B U(1)$ and let $\xi: \Omega \Sigma\left(\mathbb{C} P^{\infty}\right) \rightarrow B U$ be the extension to a loop map. The point of view in Baker and Richter [2] is that $\Omega \Sigma\left(\mathbb{C} P^{\infty}\right)$ is a topological model for the ring of quasisymmetric functions in the sense that the latter may be identified with the integral cohomology ring $H^{*}\left(\Omega \Sigma\left(\mathbb{C} P^{\infty}\right)\right)$. On cohomology $\xi$ then corresponds to the inclusion of the symmetric functions in the ring of quasisymmetric functions. Based on Theorem 1, the authors deduce in [2] that $\operatorname{THH}(T(\xi))$ may be identified with the Thom spectrum of the map

$$
L\left(\Sigma \mathbb{C} P^{\infty}\right) \longrightarrow L(B B U) \simeq B U \times B B U \stackrel{\text { proj }}{\longrightarrow} B U .
$$

This uses that $\eta: B B U \rightarrow B U$ is null homotopic. In particular, the spectrum homology of $\operatorname{THH}(T(\xi))$ is isomorphic to the homology of $L\left(\Sigma \mathbb{C} P^{\infty}\right)$.

\section{The strategy for analyzing $\operatorname{THH}(T(f))$}

We here begin to explain the ideas and constructions going into the proof of the main results. Let $\left(\mathcal{A}, \otimes, 1_{\mathcal{A}}\right)$ be a symmetric monoidal category. Recall that if $A$ is a monoid in $\mathcal{A}$, then the cyclic bar construction is the cyclic object $B_{\bullet}^{\text {cy }}(A):[k] \mapsto A^{\bigotimes(k+1)}$, with face operators

$$
d_{i}\left(a_{0} \otimes \cdots \otimes a_{k}\right)= \begin{cases}a_{0} \otimes \cdots \otimes a_{i} a_{i+1} \otimes \cdots \otimes a_{k}, & i=0, \ldots k-1, \\ a_{k} a_{0} \otimes \cdots \otimes a_{k-1}, & i=k,\end{cases}
$$


degeneracy operators

$$
s_{i}\left(a_{0} \otimes \cdots \otimes a_{k}\right)=a_{0} \otimes \ldots a_{i} \otimes 1_{\mathcal{C}} \otimes a_{i+1} \cdots \otimes a_{k}, \quad i=0, \ldots, k,
$$

and cyclic operators

$$
t_{k}\left(a_{0} \otimes \cdots \otimes a_{k}\right)=a_{k} \otimes a_{0} \otimes \cdots \otimes a_{k-1} .
$$

Here the notation is supposed to be self-explanatory. We denote the geometric realization of this object (when this notion makes sense) as $B^{\mathrm{cy}}(A)$. When $\mathcal{A}$ is one of the modern symmetric monoidal categories of spectra and $T$ is a ring spectrum (ie, a monoid in $\mathcal{A}$ ), then $B^{\text {cy }}(T)$ is a model of the topological Hochschild homology provided that $T$ satisfies suitable cofibrancy condition; see Elmendorf, Kriz, Mandell and May [16] and Shipley [46].

Suppose now that $f: A \rightarrow B F$ is a loop map that has been rectified to a map of topological monoids and imagine temporarily that $B F$ could be realized as a commutative topological monoid. Then associated to $f$ we would have the simplicial map

$$
B_{\bullet}^{\mathrm{cy}}(f): B_{\bullet}^{\mathrm{cy}}(A) \longrightarrow B_{\bullet}^{\mathrm{cy}}(B F) \longrightarrow B F,
$$

where $B F$ is viewed as a constant simplicial space and the last map is given by levelwise multiplication. The intuitive picture underlying our results is the notion that the Thom spectrum functor should take the cyclic bar construction in spaces to the cyclic bar construction in spectra in the sense that $T\left(B^{\text {cy }}(f)\right)$ should be stably equivalent to $B^{\text {cy }}(T(f))$. Ignoring issues of cofibrancy, this exactly gives a description of THH $(T(f))$ in terms of a Thom spectrum. This picture makes contact with the free loop space in the following fashion: When $A$ is a topological monoid $B^{\text {cy }}(A)$ inherits an action of the circle group $\mathbb{T}$ from the cyclic structure. This gives rise to the composite map

$$
\mathbb{T} \times B^{\mathrm{cy}}(A) \longrightarrow B^{\mathrm{cy}}(A) \longrightarrow B(A),
$$

where $B(A)$ is the realization of the usual bar construction and the second map is the realization of the simplicial map

$$
\left(a_{0}, \ldots, a_{k}\right) \mapsto\left(a_{1}, \ldots, a_{k}\right) .
$$

The adjoint map $B^{\text {cy }}(A) \rightarrow L(B(A))$ fits in a commutative diagram of spaces

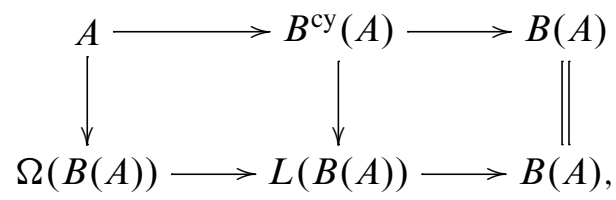


where the vertical map on the left is the usual group-completion. Standard results on realizations of simplicial quasifibrations imply that if $A$ is grouplike ( $\pi_{0} A$ is a group) and well-based (the inclusion of the unit is a Hurewicz cofibration), then the upper sequence is a fibration sequence up to homotopy and the vertical maps are weak homotopy equivalences (see for instance the proof of Lemma V.I.3 of Goodwillie [18]). This idea of relating the cyclic bar construction to the free loop space is originally due to Waldhausen [50] and suggests that we should be able to connect the description in terms of the cyclic bar construction to the free loop space.

Furthermore, if the topological monoid $A$ is in fact an infinite loop space, then there is a canonical splitting $B^{\text {cy }}(A) \rightarrow A$ of the upper sequence and it is proved by the third author [40] that the composition

$$
B(A) \longrightarrow L(B(A)) \stackrel{\simeq}{\longleftarrow} B^{\mathrm{cy}}(A) \longrightarrow A
$$

represents multiplication by the Hopf map $\eta$. Applied to $B F$ this is the essential reason why $\eta$ appears in the statement of our main theorem.

However, there are formidable technical impediments to making this intuitive picture precise. For one thing, $B F$ cannot be realized as a commutative topological monoid since it is not a generalized Eilenberg-Mac Lane space. Moreover, the classical comparison of the Thom spectrum of a cartesian product to the smash product of the Thom spectra is insufficiently rigid; one obtains a simplicial map relating $B_{\bullet}^{\mathrm{cy}}(T(f))$ and $T\left(B_{\bullet}^{\text {cy }}(f)\right)$ only after passage to the stable homotopy category. This is not sufficient for computing the topological Hochschild homology.

A major part of this paper is concerned with developing suitable technical foundations to carry out the program above. Our approach is as follows: We introduce a symmetric monoidal category $\left(\mathcal{A}, \otimes, 1_{\mathcal{A}}\right)$ which is a refined model of the category $\mathcal{U}$ of unbased spaces in the sense that $E_{\infty}$ objects can be realized as strictly commutative $\nabla$-monoids. In particular, $B F$ will admit a model as a commutative $\otimes$-monoid $B F_{\mathcal{A}}$. In this setting we show that the Thom spectrum functor can be refined to a strong symmetric monoidal functor $T_{\mathcal{A}}: \mathcal{A} / B F_{\mathcal{A}} \rightarrow \mathcal{S}$, where $\mathcal{S}$ is a suitable symmetric monoidal category of spectra. Here $\mathcal{A} / B F_{\mathcal{A}}$ denotes the category of objects in $\mathcal{A}$ over $B F_{\mathcal{A}}$ with the symmetric monoidal structure inherited from $\mathcal{A}$ : given two objects $f: X \rightarrow B F_{\mathcal{A}}$ and $g: Y \rightarrow B F_{\mathcal{A}}$, the monoidal product is defined by

$$
f \otimes g: X \otimes Y \longrightarrow B F_{\mathcal{A}} \otimes B F_{\mathcal{A}} \longrightarrow B F_{\mathcal{A}} .
$$

This is symmetric monoidal precisely because $B F_{\mathcal{A}}$ is commutative. That $T_{\mathcal{A}}$ is strong monoidal means that there is a natural isomorphism

$$
T_{\mathcal{A}}(f) \wedge T_{\mathcal{A}}(g) \stackrel{\cong}{\rightrightarrows} T_{\mathcal{A}}(f \otimes g)
$$


and this implies that we can directly implement the intuitive strategy discussed above. Of course, there is significant technical work necessary to retain homotopical control over the quantities involved in the formula above, but the basic approach does become as simple as indicated.

We construct two different realizations of the category $\mathcal{A}$. In a precise sense, our constructions herein are space-level analogues of the constructions of the modern symmetric monoidal categories of spectra. Just as there is an operadic approach to a symmetric monoidal category of spectra given by [16] and a "diagrammatic" approach given by (for example) symmetric spectra [20; 31], we have operadic and diagrammatic approaches to producing $\mathcal{A}$. Since there are several good choices for the category $\mathcal{A}$, we shall in fact give an axiomatic description of the properties needed to prove Theorem 1. The point is that even though these settings are in some sense equivalent, the natural input for the respective Thom spectrum functors is very different. Working in an axiomatic setting gives a flexible framework for adapting the constructions to fit the input provided in particular cases. Both of our realizations are based in part on the earlier constructions of May, Quinn and Ray [34] and Lewis and May [23].

\section{$\mathcal{L}(1)$-spaces and $S$-modules}

Our first construction is intimately related to the $S$-modules of [16]. Let $\mathcal{L}(n)$ denote the space of linear isometries $\mathcal{L}\left(\mathbb{U}^{n}, \mathbb{U}\right)$, for a fixed countably infinite-dimensional real inner product space $\mathbb{U}$. The object $\mathcal{L}(1)$ is a monoid under composition, and we consider the category of $\mathcal{L}(1)$-spaces. Following the approach of Kriz and May [22], we construct an "operadic smash product" on this category of spaces defined as the coequalizer

$$
X \otimes Y=\mathcal{L}(2) \times_{\mathcal{L}(1) \times \mathcal{L}(1)}(X \times Y) .
$$

This product has the property that an $A_{\infty}$ space is a monoid and an $E_{\infty}$ space is a commutative monoid. Therefore $B F$ is a commutative monoid with respect to the $\triangle$ product, and so we can adapt the Lewis-May Thom spectrum functor to construct a Thom spectrum functor from a certain subcategory of $\mathcal{L}(1)$-spaces over $B F$ to $S$-modules which is strong symmetric monoidal. The observation that one could carry out the program of [16] in the setting of spaces is due to Mike Mandell, and was worked out in the thesis of the first author [6].

\section{$\mathcal{I}$-spaces and symmetric spectra}

Our second construction is intimately related to the symmetric spectra of $[20 ; 31]$. Let $\mathcal{I}$ be the category with objects the finite sets $\mathbf{n}=\{1, \ldots, n\}$ and morphisms the injective maps. The empty set $\mathbf{0}$ is an initial object. The usual concatenation $\mathbf{m} \sqcup \mathbf{n}$ of 
finite ordered sets makes this a symmetric monoidal category with symmetric structure maps $\mathbf{m} \sqcup \mathbf{n} \rightarrow \mathbf{n} \sqcup \mathbf{m}$ given by the obvious shuffles. By definition, an $\mathcal{I}$-space is a functor $X: \mathcal{I} \rightarrow \mathcal{U}$, where $\mathcal{U}$ is the category of unbased spaces, and we write $\mathcal{I U}$ for the category of such functors. Just as for the diagrammatic approach to the smash product of spectra, this category inherits a symmetric monoidal structure from $\mathcal{I}$ via the left Kan extension

$$
(X \otimes Y)(n)=\operatorname{colim}_{\mathbf{n}_{1} \sqcup \mathbf{n}_{2} \rightarrow \mathbf{n}} X\left(n_{1}\right) \times Y\left(n_{2}\right)
$$

along the concatenation functor $\sqcup: \mathcal{I}^{2} \rightarrow \mathcal{I}$. The unit for the monoidal product is the constant $\mathcal{I}$-space $\mathcal{I}(\mathbf{0},-)$ which we denote by $*$. As we recall in Section 2.1, the correspondence $B F: \mathbf{n} \mapsto B F(n)$ defines a commutative monoid in $\mathcal{I U}$. We can therefore adapt the usual levelwise Thom space functor to construct a strong symmetric monoidal Thom spectrum functor from the category $\mathcal{I U} / B F$ to the category of symmetric spectra. This point of view on Thom spectra has been worked out in detail by the third author [42]. A similar construction applies to give a strong symmetric monoidal Thom spectrum functor with values in orthogonal spectra.

Acknowledgments The authors are grateful to a number of mathematicians for helpful conversations related to this work, including Gunnar Carlsson, Mike Hopkins, Wuchung Hsiang, Ib Madsen, Mike Mandell, Peter May, Irakli Patchkoria and Steffen Sagave. The authors would also like to thank an anonymous referee for detailed comments and suggestions based on a very careful reading of a previous draft. The first author was partially supported by an NSF postdoctoral fellowship and the second author was partially supported by a grant from the NSF.

\section{Organization of the paper}

In Section 2 we begin by reviewing the construction of the Lewis-May Thom spectrum functor. We then set up an axiomatic framework which specifies the properties of a rigid Thom spectrum functor needed to prove Theorem 1. Following this, in Section 3, we show how our main theorems can be deduced from these axioms. The rest of the paper is devoted to implementations of the axiomatic framework. We collect the relevant background material for the $S$-module approach in Section 4 and verify the axioms in this setting in Section 5. In Section 6 we discuss modifications needed to work in the context of universal quasifibrations. We then switch gears and consider the setting of $\mathcal{I}$-spaces and symmetric spectra. In Section 7 we collect and formulate some background material on symmetric spectra and we verify the axioms in this setting in Section 8. Finally, we discuss the technical details of the passage from loop maps to maps structured by operads in Appendix A. 


\section{Thom spectrum functors}

In this section we formalize the properties of a rigid Thom spectrum functor needed in order to prove our main theorems. We begin by reviewing the details of the underlying Thom spectrum functor, roughly following Lewis [23, IX]. Our rigid Thom spectrum functors are built on this foundation and this construction provides the "reference" homotopy type we expect from a Thom spectrum functor.

\subsection{The Lewis-May Thom spectrum functor}

We work in the categories $\mathcal{U}$ and $\mathcal{T}$ of based and unbased compactly generated weak Hausdorff spaces. By a spectrum $E$ we understand a sequence of based spaces $E_{n}$ for $n \geq 0$, equipped with a sequence of structure maps $S^{1} \wedge E_{n} \rightarrow E_{n+1}$ (this is what is called a prespectrum in [23]). We write $S p$ for the category of spectra in which a morphism is a sequence of maps that strictly commute with the structure maps. Let $F(n)$ be the topological monoid of base point preserving homotopy equivalences of $S^{n}$. Following Lewis we use the notation $B F(n)$ for the usual bar construction $B(*, F(n), *)$ and $E F(n)$ for the one-sided bar construction $B\left(*, F(n), S^{n}\right)$. The map $E F(n) \rightarrow B F(n)$ induced by the projection $S^{n} \rightarrow *$ is a quasifibration with fiber $S^{n}$ over each point in the base, and the inclusion of the basepoint in $S^{n}$ defines a section which is a Hurewicz cofibration. We refer the reader to Lewis, May, Steinberger and McClure [23] and May [33] for more details of these constructions. The Thom space of a map $f: X \rightarrow B F(n)$ is defined to be the quotient space

$$
T(f)=f^{*} E F(n) / X,
$$

where $f^{*} E F(n)$ is the pullback of $E F(n)$ and $X$ is viewed as a subspace via the induced section. Let $B F$ be the colimit of the spaces $B F(n)$ under the natural inclusions. Given a map $f: X \rightarrow B F$, we define a filtration of $X$ by letting $X(n)$ be the closed subspace $f^{-1} B F(n)$. The Thom spectrum $T(f)$ then has as its $n$-th space the Thom space $T\left(f_{n}\right)$, where $f_{n}$ denotes the restriction of $f$ to a map $X(n) \rightarrow B F(n)$. The structure maps are induced by the pullback diagrams

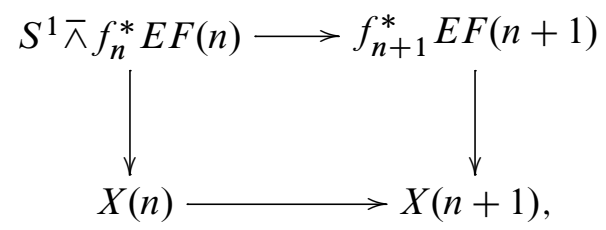


where here $\bar{\wedge}$ is the fibre-wise smash product. This construction defines our Thom spectrum functor

$$
T: \mathcal{U} / B F \longrightarrow \mathrm{Sp}
$$

We next recall how to extend this construction to a functor with values in the category $\mathscr{Y}$ of coordinate-free spectra from [23]. Thus, we consider spectra indexed on the finite dimensional subspaces $V$ of an inner product space $\mathbb{U}$ of countable infinite dimension. Let $F(V)$ be the topological monoid of base point preserving homotopy equivalences of the one-point compactification $S^{V}$. As in the case of $S^{n}$ there is an associated quasifibration $E F(V) \rightarrow B F(V)$ with fiber $S^{V}$. We again write $B F$ for the colimit over the inclusions of the spaces $B F(V)$ and observe that this is homeomorphic to the space $B F$ consider above. Given a map $f: X \rightarrow B F$, let $X(V)$ be the closed subset $f^{-1} B F(V)$ and let $T(f)(V)$ be the Thom space of the induced map $f_{V}: X(V) \rightarrow$ $B F(V)$. This defines an object $T(f)$ in the category $\mathscr{P}$ of coordinate-free prespectra from [23] and composition with the spectrification functor $L: \mathscr{P} \rightarrow \mathscr{S}$ gives the Thom spectrum functor

$$
T_{\mathscr{S}}: \mathcal{U} / B F \longrightarrow \mathscr{S}
$$

from [23]. It is an important point of the present paper that these Thom spectrum functors can be rigidified to strong monoidal functors by suitable modifications of the domains and targets. This is based on the fact [7; 34], that the correspondence $V \mapsto B F(V)$ defines a (lax) symmetric monoidal functor (see eg Mac Lane [25]) from the category $\mathcal{V}$ of finite dimensional inner product spaces and linear isometries to the category $\mathcal{U}$ of spaces. The monoidal structure maps

$$
B F(V) \times B F(W) \longrightarrow B F(V \oplus W)
$$

are induced by the monoid homomorphisms that map a pair of equivalences to their smash product. Here we implicitly use that the bar construction preserves products. There are now two ways in which this multiplicative structure leads to a "representation" of the space $B F$ as a strictly commutative monoid. On the one hand it follows from $[34,1.1 .6]$ that $B F$ has an action of the linear isometries operad and we shall see in Section 4 that this implies that it defines a commutative monoid in the weak symmetric monoidal category of $\mathcal{L}(1)$-spaces. On the other hand, the category of functors from $\mathcal{V}$ to $\mathcal{U}$ is itself a symmetric monoidal category and the (lax) symmetric monoidal structure of the functor $V \mapsto B F(V)$ exactly corresponds to a commutative monoid structure in this functor category. Composing with the functor $\mathcal{I} \rightarrow \mathcal{V}$ that maps a finite set to the vector space it generates we get a "representation" of $B F$ as a commutative monoid in the category of $\mathcal{I}$-spaces. 
More generally, one may consider $\mathcal{V}$-diagrams of topological monoids $V \mapsto G(V)$ equipped with a unital, associative, and commutative natural pairing

$$
G(V) \times G(W) \longrightarrow G(V \oplus W) .
$$

After applying the bar construction this gives a (lax) symmetric monoidal functor $V \mapsto$ $B G(V)$. For instance, we have the (lax) symmetric monoidal functors $V \mapsto B O(V)$ defined by the orthogonal groups and $V \mapsto B \operatorname{Top}(V)$ defined by the groups $\operatorname{Top}(V)$ of base point preserving homeomorphisms of $S^{V}$. We write $B G$ for the colimit of the spaces $B G(V)$ and as in the case of $B F$ this can be "represented" as a strictly commutative monoid both in an "operadic" and a "diagrammatic" fashion. Given a natural transformation of $\mathcal{V}$-diagrams of monoids $G(V) \rightarrow F(V)$, we define the Thom spectrum functor on $\mathcal{U} / B G$ to be the composition

$$
T: \mathcal{U} / B G \rightarrow \mathcal{U} / B F \stackrel{T}{\rightarrow} \text { Sp, respectively } T_{\mathscr{S}}: \mathcal{U} / B G \rightarrow \mathcal{U} / B F \stackrel{T_{\mathscr{S}}}{\rightarrow} \mathscr{S} .
$$

Finally, we must comment on homotopy invariance. Due to the fact that quasifibrations and cofibrations are not in general preserved under pullback, the Thom spectrum functor is not a homotopy functor on the whole category $\mathcal{U} / B F$. A good remedy for this is to functorially replace an object $f$ by a Hurewicz fibration $\Gamma(f): \Gamma_{f}(X) \rightarrow B F$ in the usual way. It then follows from [23, IX, 4.9] that the composite functor

$$
T \Gamma: \mathcal{U} / B F \stackrel{\Gamma}{\rightarrow} \mathcal{U} / B F \stackrel{T}{\rightarrow} \mathrm{Sp}
$$

is a homotopy functor in the sense that it takes weak homotopy equivalences over $B F$ to stable equivalences. Given a map $f: X \rightarrow B F$, there is a natural homotopy equivalence $X \rightarrow \Gamma_{f}(X)$, which we may view as a natural transformation from the identity functor on $\mathcal{U} / B F$ to $\Gamma$. We think of $T(f)$ as representing the "correct" homotopy type if the induced map $T(f) \rightarrow T(\Gamma(f))$ is a stable equivalence, and in this case we say that $f$ is $T$-good. It follows from the above discussion that the restriction of $T$ to the full subcategory of $T$-good objects is a homotopy functor. Thus, for the statement in Theorem 1 to be homotopically meaningful we have tacitly chosen $T$-good representatives before applying the Thom spectrum functor. We say that a $\mathcal{V}$-diagram of monoids $V \mapsto G(V)$ is group valued if each of the monoids $G(V)$ is a group. In this case the homotopical analysis of the Thom spectrum functor simplifies since $\mathcal{U} / B G$ maps into the subcategory of $T-\operatorname{good}$ objects in $\mathcal{U} / B F$. The Thom spectrum functor on $\mathcal{U} / B G$ is therefore a homotopy functor if $G$ is group valued.

In the following we describe in an axiomatic manner the properties required of a rigid Thom spectrum functor in order to prove Theorem 1. For simplicity we only formulate these axioms for maps over $B G$ when $G$ is group valued. One may also formulate such axioms in the general case by repeated use of the functor $\Gamma$, but we feel that 
this added technicality would obscure the presentation. In the implementation of the axioms in Section 5 and Section 8 we discuss how to modify the constructions so as to obtain Theorem 1 in general.

\subsection{Rigid Thom spectrum functors}

Let $\mathcal{S}$ denote a symmetric monoidal category of "spectra". The reader should have in mind for instance the categories of $S$-modules [16] or topological symmetric spectra [31]. Formally, we require that $\mathcal{S}$ be a symmetric monoidal topological category and that there is a continuous functor $U: \mathcal{S} \rightarrow \mathrm{Sp}$, which we think of as a forgetful functor. A morphism in $\mathcal{S}$ is said to be a weak equivalence if the image under $U$ is a stable equivalence of spectra. (In the case of topological symmetric spectra the "forgetful" functor we use is the composite of the forgetful functor and Shipley's detection functor [46]; see Section 7.1 for details).

We further require that $\mathcal{S}$ be cocomplete and tensored over unbased spaces. As we recall in Section 3.1, this implies that there is an internal notion of geometric realization for simplicial objects in $\mathcal{S}$. We also assume that the category of monoids in $\mathcal{S}$ comes equipped with a full subcategory whose objects we call flat monoids. Given a flat monoid in $\mathcal{S}$, we define its topological Hochschild homology to be the geometric realization of the cyclic bar construction. (In the implementations, the flat objects are sufficiently "cofibrant" for this to represent the correct homotopy type.)

We write $\mathcal{A}$ for our refined category of spaces. Formally, we require that $\mathcal{A}$ be a closed symmetric monoidal topological category with monoidal product $\square$ and unit $1_{\mathcal{A}}$, and we assume that there is a continuous functor $U: \mathcal{A} \rightarrow \mathcal{U}$ which we again think of as a forgetful functor. A morphism in $\mathcal{A}$ is said to be a weak equivalence if the image under $U$ is a weak homotopy equivalence of spaces. We also require that $\mathcal{A}$ be cocomplete and tensored over unbased spaces and that $U$ preserves colimits and tensors.

The following list of Axioms (A1)-(A6) specifies the properties we require of a rigid Thom spectrum functor. Here $B G$ denotes the colimit of a (lax) symmetric monoidal $\mathcal{V}$-diagram $V \mapsto B G(V)$ as discussed in Section 2.1 and we assume that $G$ is group valued.

(A1) There exists a commutative monoid $B G_{\mathcal{A}}$ in $\mathcal{A}$ and a weak homotopy equivalence $\zeta: B G_{U} \stackrel{\simeq}{\rightarrow} B G$, where $B G_{U}$ denotes the image of $B G_{\mathcal{A}}$ under the functor $U$. We further assume that $B G_{\mathcal{A}}$ is augmented in the sense that there is a map of monoids $B G_{\mathcal{A}} \rightarrow 1_{\mathcal{A}}$. 
(A2) There exists a strong symmetric monoidal "Thom spectrum" functor

$$
T_{\mathcal{A}}: \mathcal{A} / B G_{\mathcal{A}} \longrightarrow \mathcal{S}
$$

that preserves weak equivalences, and commutes with colimits and tensors with unbased spaces. We require that $T_{\mathcal{A}}$ be a lift of the Lewis-May Thom spectrum functor on $\mathcal{U} / B G$ in the sense that the two compositions in the diagram

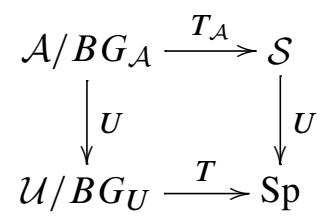

are related by a chain of natural stable equivalences. Here $T$ denotes the composite functor

$$
\mathcal{U} / B G_{U} \stackrel{\zeta_{*}}{\rightarrow} \mathcal{U} / B G \stackrel{T}{\rightarrow} \mathrm{Sp} .
$$

These two axioms already guarantee that we can carry out the argument sketched in the introduction. Let $\alpha: A \rightarrow B G_{\mathcal{A}}$ be a monoid morphism, and let $B^{\text {cy }}(\alpha)$ be the realization of the simplicial map

$$
B_{\bullet}^{\mathrm{cy}}(\alpha): B_{\bullet}^{\mathrm{cy}}(A) \longrightarrow B_{\bullet}^{\mathrm{cy}}\left(B G_{\mathcal{A}}\right) \longrightarrow B G_{\mathcal{A}},
$$

where we view $B G_{\mathcal{A}}$ as a constant simplicial object.

Theorem 2.1 Let $\alpha: A \rightarrow B G_{\mathcal{A}}$ be a monoid morphism in $\mathcal{A}$. Then $T_{\mathcal{A}}(\alpha)$ is a monoid in $\mathcal{S}$ and there is an isomorphism

$$
B^{\mathrm{cy}}\left(T_{\mathcal{A}}(\alpha)\right) \cong T_{\mathcal{A}}\left(B^{\mathrm{cy}}(\alpha)\right) .
$$

Furthermore, there is a stable equivalence

$$
U T_{\mathcal{A}}\left(B^{\mathrm{cy}}(\alpha)\right) \stackrel{\simeq}{\rightarrow} T\left(U B^{\mathrm{cy}}(\alpha)\right) .
$$

The simplicity of this result, once we have set up the framework of the two axioms, is very satisfying. However, since we are really interested in topological Hochschild homology, we must be able to represent our Thom spectra as flat monoids in $\mathcal{S}$ and for this reason we introduce the functor $C$ below. This should be thought of as a kind of cofibrant replacement functor and for the application of Theorem 2.1 it is essential that this "replacement" takes place in the category $\mathcal{A}$ before applying $T$. Adapting the usual convention for topological monoids to our setting, we say that a monoid in $\mathcal{A}$ is well-based if the unit $1_{\mathcal{A}} \rightarrow A$ has the homotopy extension property; see Section 3.1 for details. We write $\mathcal{A}[\mathbb{T}]$ for the category of monoids in $\mathcal{A}$. 
(A3) There exists a functor

$$
C: \mathcal{A}[\mathbb{T}] \longrightarrow \mathcal{A}[\mathbb{T}], \quad A \mapsto A^{c},
$$

and a natural weak equivalence $A^{c} \rightarrow A$ in $\mathcal{A}[\mathbb{T}]$. We require that the monoid $A^{c}$ be well-based and that the composite functor

$$
\mathcal{A}[\mathbb{T}] / B G_{\mathcal{A}} \stackrel{C}{\rightarrow} \mathcal{A}[\mathbb{T}] / B G_{\mathcal{A}} \stackrel{T_{\mathcal{A}}}{\longrightarrow} \mathcal{S}, \quad \alpha \mapsto T_{\mathcal{A}}\left(A^{c} \longrightarrow A \stackrel{\alpha}{\rightarrow} B G_{\mathcal{A}}\right)
$$

takes values in the full subcategory of flat monoids in $\mathcal{S}$.

As explained earlier, we think of the symmetric monoidal category $\mathcal{A}$ as a refined model of the category of spaces in which we can represent $E_{\infty}$ monoids by strictly commutative monoids. Whereas the functor $U$ should be thought of as a forgetful functor, the functor $Q$ introduced below encodes the relationship between the monoidal product $\otimes$ and the cartesian product of spaces.

(A4) There exists a strong symmetric monoidal functor $Q: \mathcal{A} \rightarrow \mathcal{U}$ that preserves colimits and tensors with unbased spaces. We further assume that there is a natural transformation $U \rightarrow Q$ that induces a weak homotopy equivalence

$$
U\left(A_{1}^{c} \otimes \cdots \otimes A_{k}^{c}\right) \longrightarrow Q\left(A_{1}^{c} \otimes \cdots \otimes A_{k}^{c}\right)
$$

for all $k \geq 0$ and all $k$-tuples of monoids $A_{1}, \ldots, A_{k}$.

For $k=0$, the last requirement amounts to the condition that $U\left(1_{\mathcal{A}}\right) \rightarrow *$ be a weak homotopy equivalence. Until now we have not made any assumptions on the homotopical behavior of $B G_{\mathcal{A}}$ with respect to the monoidal structure. The next axiom ensures that we may replace $B G_{\mathcal{A}}$ by a commutative monoid which is in a certain sense well-behaved.

(A5) There exists a well-based commutative monoid $B G_{\mathcal{A}}^{\prime}$ in $\mathcal{A}$ and a weak equivalence of monoids $B G_{\mathcal{A}}^{\prime} \rightarrow B G_{\mathcal{A}}$. We assume that the canonical map (induced by the augmentation)

$$
U(\underbrace{B G_{\mathcal{A}}^{\prime} \otimes \cdots \otimes B G_{\mathcal{A}}^{\prime}}_{k}) \longrightarrow \underbrace{U B G_{\mathcal{A}}^{\prime} \times \cdots \times U B G_{\mathcal{A}}^{\prime}}_{k}
$$

is an equivalence for all $k$.

Since the functor $Q$ is monoidal it takes monoids in $\mathcal{A}$ to ordinary topological monoids. We say that a monoid $A$ in $\mathcal{A}$ is grouplike if the topological monoid $Q A^{c}$ is grouplike in the ordinary sense. Now let $\alpha: A \rightarrow B G_{\mathcal{A}}$ be a monoid morphism and let us write $X=U A$ and $f=U \alpha$. We define $B X$ and $B^{2} G_{U}$ to be the realizations of the 
simplicial spaces $U B_{\bullet}\left(A^{c}\right)$ and $U B \bullet\left(B G_{\mathcal{A}}^{c}\right)$, and we define $B f$ to be the realization of the simplicial map induced by $\alpha$, that is

$$
B f=U B\left(\alpha^{c}\right): B X=\left|U B_{\bullet}\left(A^{c}\right)\right| \longrightarrow\left|U B_{\bullet}\left(B G_{\mathcal{A}}^{c}\right)\right|=B^{2} G_{U} .
$$

We shall see in Section 3.2 that (A3) and (A4) imply that this is a delooping of $f$ if $A$ is grouplike.

Theorem 2.2 Suppose that (A1)-(A5) hold and that $A$ is grouplike. Then there is a stable equivalence

$$
T\left(U B^{\mathrm{cy}}\left(\alpha^{c}\right)\right) \simeq T\left(L^{\eta}(B f)\right),
$$

where $L^{\eta}(B f)$ is the map

$$
L(B X) \stackrel{L(B f)}{\longrightarrow} L\left(B^{2} G_{U}\right) \simeq B G_{U} \times B^{2} G_{U} \stackrel{\text { id } \times \eta}{\longrightarrow} B G_{U} \times B G_{U} \longrightarrow B G_{U},
$$

defined as in Theorem 1.

Combining this result with Theorem 2.1, we get a stable equivalence

$$
U B^{\mathrm{cy}}\left(T_{\mathcal{A}}\left(\alpha^{c}\right)\right) \simeq T\left(L^{\eta}(B f)\right)
$$

and since $T_{\mathcal{A}}\left(\alpha^{c}\right)$ is a flat replacement of $T_{\mathcal{A}}(\alpha)$ by assumption, this gives an abstract version of Theorem 1. In order to obtain the latter, we must be able to lift space level data to $\mathcal{A}$. This is the purpose of our final axiom.

(A6) There exists an $A_{\infty}$ operad $\mathcal{C}_{\mathcal{A}}$ that acts on $B G_{U}$ and a functor

$$
R: \mathcal{U}\left[\mathcal{C}_{\mathcal{A}}\right] / B G_{U} \longrightarrow \mathcal{A}[\mathbb{T}] / B G_{\mathcal{A}}, \quad\left(X \stackrel{f}{\rightarrow} B G_{U}\right) \mapsto\left(R_{f}(X) \stackrel{R(f)}{\longrightarrow} B G_{\mathcal{A}}\right),
$$

where $\mathcal{U}\left[\mathcal{C}_{\mathcal{A}}\right]$ is the category of spaces with $\mathcal{C}_{\mathcal{A}}$-action. Further, we require that $R(\mathrm{id}): R_{\mathrm{id}}\left(B G_{U}\right) \rightarrow B G_{\mathcal{A}}$ is a weak equivalence and that the composite functor

$$
\mathcal{U}\left[\mathcal{C}_{\mathcal{A}}\right] / B G_{U} \stackrel{R}{\rightarrow} \mathcal{A}[\mathbb{T}] / B G_{\mathcal{A}} \longrightarrow \mathcal{A}[\mathbb{T}] \stackrel{C}{\rightarrow} \mathcal{A}[\mathbb{T}] \stackrel{Q}{\rightarrow} \mathcal{U}[\mathbb{T}] \longrightarrow \mathcal{U}\left[\mathcal{C}_{\mathcal{A}}\right]
$$

is related to the forgetful functor by a chain of natural weak homotopy equivalences in $\mathcal{U}\left[\mathcal{C}_{\mathcal{A}}\right]$.

The second arrow represents the forgetful functor and the last arrow represents the functor induced by the augmentation from the $A_{\infty}$ operad $\mathcal{C}_{\mathcal{A}}$ to the associativity operad [32]. It follows from (A3), (A4) and (A6) that there is a chain of weak homotopy equivalences relating $X$ to $U R_{f}(X)$. Thus, in this sense $R$ is a partial right homotopy inverse of $U$. We shall later see that if $X$ is grouplike, then the conditions in (A6) ensure that the delooping of $f$ implied by the operad action is homotopic to the map defined in (2-1). 


\section{Proofs of the main results from the axioms}

We first recall some background material on tensored categories and geometric realization.

\subsection{Simplicial objects and geometric realization}

Let $\mathcal{A}$ be a cocomplete topological category. Thus, we assume that $\mathcal{A}$ is enriched over $\mathcal{U}$ in the sense that the morphism sets $\mathcal{A}(A, B)$ are topologized and the composition maps continuous. The category $\mathcal{A}$ is tensored over unbased spaces if there exists a continuous functor $\otimes: \mathcal{A} \times \mathcal{U} \rightarrow \mathcal{A}$, together with a natural homeomorphism

$$
\operatorname{Map}(X, \mathcal{A}(A, B)) \cong \mathcal{A}(A \otimes X, B),
$$

where $A$ and $B$ are objects in $\mathcal{A}$ and $X$ is a space. For the category $\mathcal{U}$ itself, the tensor is given by the cartesian product, and in Sp the tensor of a spectrum $A$ with an (unbased) space $X$ is the levelwise smash product $A \wedge X_{+}$. Assuming that $\mathcal{A}$ is tensored, there is an internal notion of geometric realization of simplicial objects. Let $[p] \mapsto \Delta^{p}$ be the usual cosimplicial space used to define the geometric realization. Given a simplicial object $A_{\bullet}$ in $\mathcal{A}$, we define the realization $\left|A_{\bullet}\right|$ to be the coequalizer of the diagram

$$
\coprod_{[p] \rightarrow[q]} A_{q} \otimes \Delta^{p} \rightrightarrows \coprod_{[r]} A_{r} \otimes \Delta^{r}
$$

where the first coproduct is over the morphisms in the simplicial category and the two arrows are defined as for the realization of a simplicial space. Notice that if we view an object $A$ as a constant simplicial object, then its realization is isomorphic to $A$. In the case where $\mathcal{A}$ is the category $\mathcal{U}$, the above construction gives the usual geometric realization of a simplicial space and if $A_{\bullet}$ is a simplicial spectrum, then $\left|A_{\bullet}\right|$ is the usual levelwise realization. The following lemma is an immediate consequence of the definitions.

Lemma 3.1 Let $\mathcal{A}$ and $\mathcal{B}$ be cocomplete topological categories that are tensored over unbased spaces and let $\Psi: \mathcal{A} \rightarrow \mathcal{B}$ be a continuous functor that preserves colimits and tensors. Then $\Psi$ also preserves realization of simplicial objects in the sense that there is a natural isomorphism $\left|\Psi A_{\bullet}\right| \cong \Psi\left|A_{\bullet}\right|$.

Following [31], we say that a morphism $U \rightarrow V$ in $\mathcal{A}$ is an $h$-cofibration if the induced morphism from the mapping cylinder

$$
V \cup_{U}(U \otimes I) \longrightarrow V \otimes I
$$


admits a retraction in $\mathcal{A}$. This generalizes the usual notion of a Hurewicz cofibration in $\mathcal{U}$, that is, of a map having the homotopy extension property. Using the terminology from the space level setting as in [45, Appendix A], we say that a simplicial object in $\mathcal{A}$ is good if the degeneracy operators are $h$-cofibrations. We observe that a functor that preserves colimits and tensors as in Lemma 3.1 also preserves $h$-cofibrations. It therefore also preserves the goodness condition for simplicial objects. If $\mathcal{A}$ has a monoidal structure, then we say that a monoid $A$ is well-based if the unit $1_{\mathcal{A}} \rightarrow A$ is an $h$-cofibration.

Lemma 3.2 Let $\mathcal{A}$ be a closed symmetric monoidal topological category that is cocomplete and tensored over unbased spaces. If $A$ is a well-based monoid in $\mathcal{A}$, then the simplicial objects $B_{\bullet}(A)$ and $B_{\bullet}^{\mathrm{cy}}(A)$ are good.

Proof We claim that if $U \rightarrow V$ is an $h$-cofibration in $\mathcal{A}$, then the induced morphism $U \otimes W \rightarrow V \otimes W$ is again an $h$-cofibration for any object $W$. In order to show this we use that $\mathcal{A}$ is closed to establish a canonical isomorphism

$$
\left(V \cup_{U}(U \otimes I)\right) \otimes W \cong V \otimes W \cup_{U \otimes W}((U \otimes W) \otimes I) .
$$

Similarly, we may identify $(V \otimes I) \otimes W$ with $(V \otimes W) \otimes I$ and the claim follows. The assumption that $A$ be well-based therefore implies the statement of the lemma. $\square$

\subsection{Consequences of the axioms}

Now let $\mathcal{A}$ and $\mathcal{S}$ be as in Section 2.2, and assume that the Axioms (A1)-(A6) hold. We shall prove the consequences of the axioms stated in Section 2.2.

Proof of Theorem 2.1 If $\alpha: A \rightarrow B F_{\mathcal{A}}$ is a monoid morphism in $\mathcal{A}$, then the assumption that $T_{\mathcal{A}}$ be strong symmetric monoidal implies that we have an isomorphism of cyclic objects $B_{\bullet}^{\mathrm{cy}}\left(T_{\mathcal{A}}(\alpha)\right) \cong T_{\mathcal{A}}\left(B_{\bullet}^{\mathrm{cy}}(\alpha)\right)$. The first statement therefore follows from Lemma 3.1 since we have assumed that $T_{\mathcal{A}}$ preserves colimits and tensors. The second statement follows from the assumption that the diagram in (A2) commutes up to stable equivalence.

The following lemma is an immediate consequence of Lemma 3.2 and the assumption that $U$ and $Q$ preserve colimits and tensors.

Lemma 3.3 If $A$ is a well-based monoid in $\mathcal{A}$, then the simplicial objects $B_{\bullet}(A)$ and $B_{\bullet}^{\text {cy }}(A)$ are good and so are the simplicial spaces obtained by applying $U$ and $Q$. 
If $Z_{\bullet}$ is a simplicial object in $\mathcal{A}$ with internal realization $Z$, then it follows from Lemma 3.1 that $U Z$ is homeomorphic to the realization of the simplicial space $U Z$. obtained by applying $U$ degree-wise. If $Z_{\bullet}$ is a cyclic object, then $U Z_{\bullet}$ is a cyclic space and $U Z$ inherits an action of the circle group $\mathbb{T}$. Recall that a monoid $A$ in $\mathcal{A}$ is said to be grouplike if the topological monoid $Q A^{c}$ is grouplike in the ordinary sense.

Proposition 3.4 If $A$ is grouplike, then $U B\left(A^{c}\right)$ is a delooping of $U A$.

Proof The natural transformation in (A4) gives rise to a map of simplicial spaces $U B_{\bullet}\left(A^{c}\right) \rightarrow Q B \bullet\left(A^{c}\right)$ which is a levelwise weak homotopy equivalence by assumption. Since by Lemma 3.3 these are good simplicial spaces, it follows that the topological realization is also a weak homotopy equivalence. Furthermore, since $Q$ is strong symmetric monoidal, $Q B\left(A^{c}\right)$ is isomorphic to the classifying space of the grouplike topological monoid $Q A^{c}$, hence is a delooping of the latter. Thus, we have a chain of weak homotopy equivalences

$$
\Omega\left(U B\left(A^{c}\right)\right) \simeq \Omega\left(Q B\left(A^{c}\right)\right) \simeq \Omega\left(B\left(Q A^{c}\right)\right) \simeq Q A^{c} \simeq U A^{c} \simeq U A,
$$

where the two last equivalences are implied by (A3) and (A4).

Suppose now that $A$ and therefore also $A^{c}$ is augmented over the unit $1_{\mathcal{A}}$. We then have the following analogue of (1-2),

$$
\mathbb{T} \times U B^{\mathrm{cy}}\left(A^{c}\right) \longrightarrow U B^{\mathrm{cy}}\left(A^{c}\right) \longrightarrow U B\left(A^{c}\right),
$$

where the last arrow is defined using the augmentation.

Proposition 3.5 If $A$ is grouplike, then the adjoint map

$$
U B^{\mathrm{cy}}\left(A^{c}\right) \longrightarrow L\left(U B\left(A^{c}\right)\right)
$$

is a weak homotopy equivalence.

Proof It follows from the proof of Proposition 3.4 that there is a weak homotopy equivalence $U B\left(A^{c}\right) \rightarrow Q B\left(A^{c}\right)$ and by a similar argument we get a weak homotopy equivalence $U B^{\text {cy }}\left(A^{c}\right) \rightarrow Q B^{\text {cy }}\left(A^{c}\right)$. These maps are related by a commutative diagram

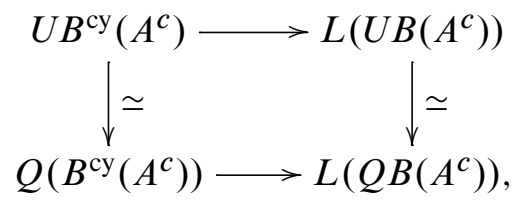


where, replacing $U$ by $Q$, the bottom map is defined as the map in the proposition. Using that $Q$ is strong symmetric monoidal, we can write the latter map in the form

$$
B^{\mathrm{cy}}\left(Q A^{c}\right) \longrightarrow L\left(B\left(Q A^{c}\right)\right),
$$

and as discussed in the introduction, this map is a weak homotopy equivalence since $Q A^{c}$ is grouplike. This implies the result.

Now let $\alpha: A \rightarrow B G_{\mathcal{A}}$ be a monoid morphism in $\mathcal{A}$ of the form considered in Theorem 2.2. We wish to analyze the map obtained by applying $U$ to the composite morphism

$$
B^{\mathrm{cy}}\left(A^{c}\right) \longrightarrow B^{\mathrm{cy}}\left(B G_{\mathcal{A}}^{c}\right) \longrightarrow B^{\mathrm{cy}}\left(B G_{\mathcal{A}}\right) \longrightarrow B G_{\mathcal{A}} .
$$

Notice first that we have a commutative diagram

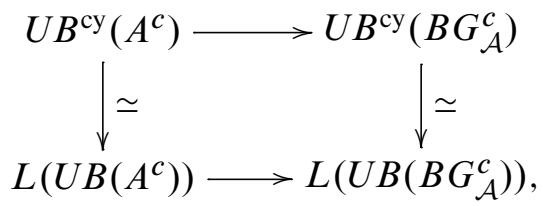

where the vertical maps are weak homotopy equivalences by Proposition 3.5. Writing $B^{2} G_{U}$ for the delooping $U B\left(B G_{\mathcal{A}}^{c}\right)$ as usual, we must identify the homotopy class represented by the diagram

$$
L\left(B^{2} G_{U}\right) \stackrel{\simeq}{\longleftarrow} U B^{\mathrm{cy}}\left(B G_{\mathcal{A}}^{c}\right) \longrightarrow U B^{\mathrm{cy}}\left(B G_{\mathcal{A}}\right) \longrightarrow B G_{U} .
$$

We shall do this by applying the results of [40] and for this we need to recall some general facts about $\Gamma$-spaces. Consider in general a commutative well-based monoid $Z$ in $\mathcal{A}$ that is augmented over the unit $1_{\mathcal{A}}$. Such a monoid gives rise to a $\Gamma$-object in $\mathcal{A}$, that is, to a functor $Z: \Gamma^{o} \rightarrow \mathcal{A}$, where $\Gamma^{o}$ denotes the category of finite based sets. It suffices to define $Z$ on the skeleton subcategory specified by the objects $\mathbf{n}_{+}=\{*, 1, \ldots, n\}$, where we let $Z\left(\mathbf{n}_{+}\right)=Z^{\bigotimes n}$ with an implicit choice of placement of the parenthesis in the iterated monoidal product. By definition, $Z\left(\mathbf{0}_{+}\right)=1_{\mathcal{A}}$. The $\Gamma$-structure is defined using the symmetric monoidal structure of $\mathcal{A}$, together with the multiplication and augmentation of $Z$. (This construction models the tensor of $Z$ with finite based sets in the category of augmented commutative monoids in $\mathcal{A}$; similar constructions are considered in $[4 ; 40]$.) From this point of view the diagram of simplicial objects

$$
Z \longrightarrow B_{\bullet}^{\mathrm{cy}}(Z) \longrightarrow B_{\bullet}(Z)
$$

may be identified with that obtained by evaluating $Z$ degree-wise on the cofibration sequence of simplicial sets $S^{0} \rightarrow S_{\bullet+}^{1} \rightarrow S_{\bullet}^{1}$; see [40, Section 5.2]. Composing with the functor $U$ we get the $\Gamma$-space $U Z$ and the assumption that the monoid $Z$ be 
well-based assures that this construction is homotopically well-behaved. Notice also that $U Z$ is degree-wise equivalent to the reduced $\Gamma$-space $\tilde{U} Z$ defined by the quotient spaces

$$
\tilde{U} Z\left(\mathbf{n}_{+}\right)=U Z\left(\mathbf{n}_{+}\right) / U\left(1_{\mathcal{A}}\right) .
$$

Following Bousfield and Friedlander [9], we say that $U Z$ is a special $\Gamma$-space if the canonical maps

$$
U(\underbrace{Z \otimes \cdots \otimes Z}_{n}) \longrightarrow \underbrace{U Z \times \cdots \times U Z}_{n}
$$

are weak homotopy equivalences for all $n$. In this case the underlying space $U Z$ inherits a weak $\mathrm{H}$-space structure, and we say that the $\Gamma$-space is very special if the monoid of components is a group. This is equivalent to the condition that $Z$ be grouplike as a monoid in $\mathcal{A}$. Consider now the composition

$$
\mathbb{T} \times U Z\left(S_{+}^{1}\right) \longrightarrow U Z\left(S_{+}^{1}\right) \longrightarrow U Z\left(S^{1}\right)
$$

defined in analogy with (1-3). The following result is an immediate consequence of $[40,7.3]$.

Proposition 3.6 [40] If $Z$ is a well-based commutative monoid in $\mathcal{A}$ such that the $\Gamma$-space $U Z$ is very special, then the adjoint map

$$
U Z\left(S_{+}^{1}\right) \stackrel{\simeq}{\rightarrow} L\left(U Z\left(S^{1}\right)\right)
$$

is a weak homotopy equivalence and the diagram of infinite loop spaces

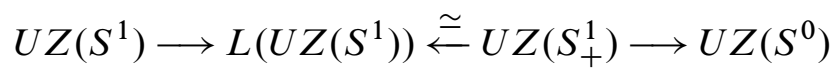

represents multiplication by $\eta$.

The last map in the above diagram is induced by the retraction $S_{+}^{1} \rightarrow S^{0}$.

Proof of Theorem 2.2 It remains to identify the homotopy class represented by (3-1) and as explained in the introduction we have a splitting

$$
L\left(B^{2} G_{U}\right) \simeq B G_{U} \times B^{2} G_{U} .
$$

We must prove that the homotopy class specified by the diagram

$$
B^{2} G_{U} \longrightarrow L\left(B^{2} G_{U}\right) \stackrel{\simeq}{\longleftarrow} U B^{\mathrm{cy}}\left(B G_{\mathcal{A}}^{c}\right) \longrightarrow B G_{U}
$$

is multiplication by $\eta$ in the sense of the theorem. Let $B G_{\mathcal{A}}^{\prime} \rightarrow B G_{\mathcal{A}}$ be as in (A5) and let the spaces $B G_{U}^{\prime}$ and $B^{2} G_{U}^{\prime}$ be defined as $U B G_{\mathcal{A}}^{\prime}$ and $U B\left(B G_{\mathcal{A}}^{c}\right)$. We then obtain a diagram

$$
B^{2} G_{U}^{\prime} \longrightarrow L\left(B^{2} G_{U}^{\prime}\right) \stackrel{\simeq}{\longleftarrow} U B^{\mathrm{cy}}\left(B G_{\mathcal{A}}^{\prime c}\right) \longrightarrow B G_{U}^{\prime}
$$


which is naturally weakly homotopy equivalent to (3-2). Writing $Z$ for the commutative monoid $B G_{\mathcal{A}}^{\prime}$, the assumptions in (A5) ensure that $Z$ gives rise to a very special $\Gamma$-space $U Z$. We claim that the diagram (3-3) is naturally weakly equivalent to the diagram in Proposition 3.6. Indeed, it follows from (A5) that the natural weak equivalence in (A3) gives rise to degree-wise weak homotopy equivalences

$$
U B_{\bullet}\left(B G_{\mathcal{A}}^{\prime}\right) \longrightarrow U Z\left(S_{\bullet}^{1}\right) \quad \text { and } \quad U B_{\bullet}^{\mathrm{cy}}\left(B G_{\mathcal{A}}^{\prime}\right) \longrightarrow U Z\left(S_{\bullet+}^{1}\right) .
$$

Since these are good simplicial spaces by Lemma 3.3, the induced maps of realizations are also weak homotopy equivalences. The statement of the theorem now follows immediately from Proposition 3.6.

\subsection{Proofs of the main theorems}

We first recall some general facts about deloopings of $A_{\infty}$ maps from [32]. Thus, let $\mathcal{C}$ be an $A_{\infty}$ operad with augmentation $\mathcal{C} \rightarrow \mathcal{M}$ where $\mathcal{M}$ denotes the associative operad. Let $C$ and $M$ be the associated monads in based spaces and consider for a $\mathcal{C}$-space $X$ the diagram of weak homotopy equivalences of $\mathcal{C}$-spaces

$$
X \stackrel{\simeq}{\longleftarrow} B(C, C, X) \stackrel{\simeq}{\longrightarrow} B(M, C, X)
$$

defined as in $[32,13.5]$. The $\mathcal{C}$-space $B(M, C, X)$ is in fact a topological monoid and we define $B^{\prime} X$ to be its classifying space, defined by the usual bar construction. If $X$ is grouplike, then $B^{\prime} X$ is a delooping in the sense that $\Omega\left(B^{\prime} X\right)$ is related to $X$ by a chain of weak homotopy equivalences. This construction is clearly functorial in $X$ : given a map of $\mathcal{C}$-spaces $X \rightarrow Y$, we have an induced map $B^{\prime} X \rightarrow B^{\prime} Y$. Now let $f: X \rightarrow B G_{U}$ be a $\mathcal{C}_{\mathcal{A}}$-map and let $\alpha: A \rightarrow B G_{\mathcal{A}}$ be the object in $\mathcal{A}[\mathbb{T}] / B G_{\mathcal{A}}$ obtained by applying the functor $R$. We let $B f: B X \rightarrow B^{2} G_{U}$ be defined as in (2-1), where we recall that $B X$ and $B^{2} G_{U}$ denote the spaces $U B\left(A^{c}\right)$ and $U B\left(B G_{\mathcal{A}}^{c}\right)$. The first step in the proof of Theorem 1 is to compare the maps $B f$ and $B^{\prime} f$.

Lemma 3.7 There is a commutative diagram

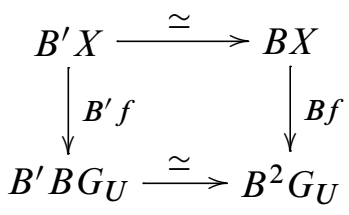

in which the horizontal arrows represent chains of compatible weak homotopy equivalences. 
Proof By definition, $A$ is the monoid $R_{f}(X)$ and it follows from (A6) that there is a chain of natural weak equivalences of $\mathcal{C}_{\mathcal{A}}$-spaces relating $X$ to $Q R_{f}(X)^{c}$. Applying the bar construction from [32] we obtain a chain of weak equivalences of topological monoids

$$
B\left(M, C_{\mathcal{A}}, X\right) \simeq B\left(M, C_{\mathcal{A}}, Q R_{f}(X)^{c}\right) \simeq B\left(M, M, Q R_{f}(X)^{c}\right) \simeq Q R_{f}(X)^{c} .
$$

The two last equivalence comes from the fact that $Q R_{f}(X)^{c}$ is itself a topological monoid [32, 13.5(iv)]. This chain in turn gives a chain of equivalences of the classifying spaces and composing with the equivalence induced by the natural transformation $U \rightarrow Q$ we get the upper row in the diagram. In particular, applied to the identity on $B G_{U}$, this construction gives a chain of weak equivalences relating $B^{\prime}\left(B G_{U}\right)$ to $U B\left(R_{\mathrm{id}}\left(B G_{U}\right)^{c}\right)$. Furthermore, the weak equivalence $R_{\mathrm{id}}\left(B G_{U}\right) \rightarrow B G_{\mathcal{A}}$ from (A6) gives rise to a weak equivalence

$$
U B\left(R_{\mathrm{id}}\left(B G_{U}\right)^{c}\right) \longrightarrow U B\left(B G_{\mathcal{A}}^{c}\right)=B^{2} G_{U}
$$

and composing with this we get the bottom row in the diagram. It is clear from the construction that the horizontal rows are compatible as claimed.

Proof of Theorem 1 We first reformulate the theorem in a more precise form. As explained in Appendix A, we may assume that our loop map $f: X \rightarrow B G_{U}$ is a $\mathcal{C}_{\mathcal{A}^{-}}$ map with a delooping of the form $B_{1} f: B_{1} X \rightarrow B_{1} B G_{U}$, where $B_{1}$ denotes the May classifying space functor. We again write $\alpha: A \rightarrow B G_{\mathcal{A}}$ for the monoid morphism in $\mathcal{A}$ obtained by applying the functor $R$. Then $U B^{\text {cy }}\left(T_{\mathcal{A}}\left(\alpha^{c}\right)\right)$ represents the topological Hochschild homology spectrum $\operatorname{THH}(T(f))$ and it follows from Theorem 2.1 and Theorem 2.2 that there is a stable equivalence

$$
U B^{\mathrm{cy}}\left(T_{\mathcal{A}}\left(\alpha^{c}\right)\right) \simeq T\left(L^{\eta}(B f)\right)
$$

where $B f$ is defined as in (2-1). We claim that there is a homotopy commutative diagram of the form

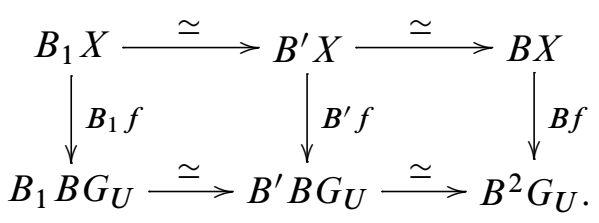

Indeed, it is proved in [49] that the functors $B_{1}$ and $B^{\prime}$ are naturally equivalent which gives the homotopy commutativity of the left hand square while the homotopy commutativity of the right hand square follows from Lemma 3.7. The result now follows from the homotopy invariance of the Thom spectrum functor. 
In preparation for the proof of Theorem 2 we recall that the Thom spectrum functor $T$ is multiplicative in the following sense: given maps $f: X \rightarrow B G_{U}$ and $g: Y \rightarrow B G_{U}$, there is a stable equivalence

$$
T(f \times g) \simeq T(f) \wedge T(g),
$$

where $f \times g$ denotes the map

$$
f \times g: X \times Y \stackrel{f \times g}{\longrightarrow} B G_{U} \times B G_{U} \longrightarrow B G_{U}
$$

defined using the $H$-space structure of $B G_{U}$. We refer to [23; 42] for different accounts of this basic fact. Of course, one of the main points of this paper is to "rigidify" this stable equivalence.

Proof of Theorem 2 As explained in the introduction, the loop space structure on $B X$ gives rise to a weak homotopy equivalence $X \times B X \stackrel{\simeq}{\rightarrow} L(B X)$. This fits in a homotopy commutative diagram

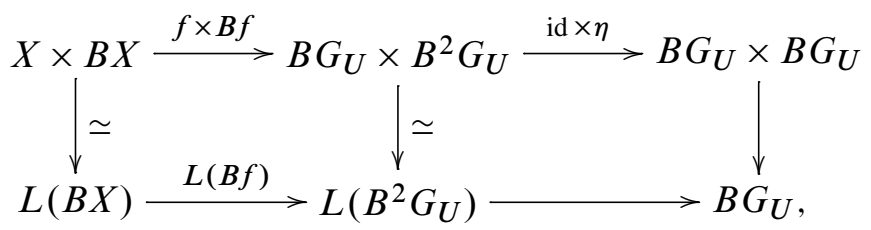

where the composition in the bottom row is the map $L^{\eta}(B f)$. By homotopy invariance of the Thom spectrum functor we get from this the stable equivalences

$$
T\left(L^{\eta}(B f)\right) \simeq T(f \times(\eta \circ B f)) \simeq T(f) \wedge T(\eta \circ B f)
$$

and the result follows from Theorem 1.

Proof of Theorem 3 Notice first that if $X$ is a 3 -fold loop space, then the unstable Hopf map $\eta$ gives rise to a map

$$
\eta: B X \simeq \operatorname{Map}_{*}\left(S^{2}, B^{3} X\right) \stackrel{\eta^{*}}{\longrightarrow} \operatorname{Map}_{*}\left(S^{3}, B^{3} X\right) \simeq X .
$$

Let $\Phi$ be the self homotopy equivalence of $X \times B X$ defined by

$$
\Phi=\left[\begin{array}{cc}
\text { id } & \eta \\
0 & \text { id }
\end{array}\right]: X \times B X \stackrel{\simeq}{\rightarrow} X \times B X
$$


Given a 3-fold loop map $f: X \rightarrow B G_{U}$, we then have a homotopy commutative diagram

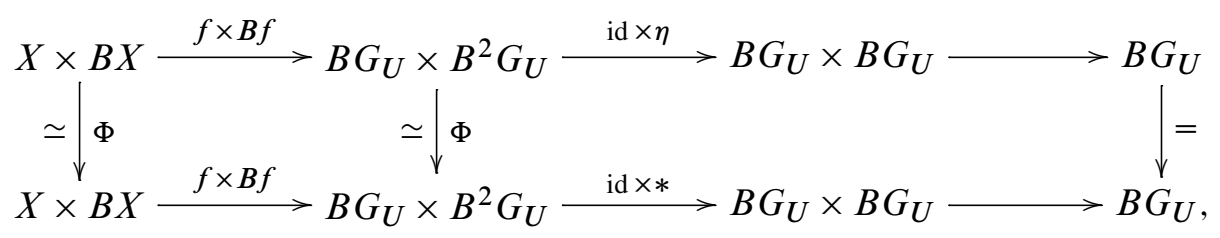

where $*$ denotes the trivial map. It follows from the proof of Theorem 2 that the composition of the maps in the upper row is weakly homotopy equivalent to $L^{\eta}(B f)$. Thus, by homotopy invariance of the Thom spectrum functor we get the stable equivalence

$$
T\left(L^{\eta}(B f)\right) \simeq T(f \times *) \simeq T(f) \wedge B X_{+}
$$

and the result again follows from Theorem 1 .

\subsection{Eilenberg-Mac Lane spectra and $p$-local Thom spectra}

In this section we first collect some background material on $p$-local Thom spectra for a prime $p$. We then complete the proofs of the theorems in the introduction calculating the topological Hochschild homology of certain Eilenberg-Mac Lane spectra. There is a $p$-local version of the Thom spectrum functor

$$
T: \mathcal{U} / B F_{(p)} \longrightarrow \mathrm{Sp}_{(p)},
$$

defined on the category of spaces over the classifying space $B F_{(p)}$ for $p$-local stable spherical fibrations and with values in the category $\operatorname{Sp}_{(p)}$ of $p$-local spectra. In fact, each of our "rigid" Thom spectrum functors has a $p$-local analogue. This is particularly easy to explain in the setting of $\mathcal{I}$-spaces and symmetric spectra: Let $S_{(p)}^{1}$ be the $p$-localization of $S^{1}$ and let $S_{(p)}^{n}$ be the $n$-fold smash product. We write $F_{(p)}(n)$ for the topological monoid of homotopy equivalences of $S_{(p)}^{n}$ and $B F_{(p)}(n)$ for its classifying space. The definition of the symmetric Thom spectrum functor can then readily be modified to a $p$-local version with values in the category of module spectra over the $p$-local sphere spectrum. A similar construction works in the $\mathcal{L}(1)$-space setting using a continuous localization functor as in [21].

It follows by inspection of our formal framework and the implementations in Section 5 and Section 8 that there are $p$-local versions of Theorems 1, 2 and 3. The application of this to the calculation of $\operatorname{THH}(H \mathbb{Z} / p)$ is as follows. Let $B F_{(p)}$ be the colimit of the spaces $B F_{(p)}(n)$ and notice that $\pi_{1} B F_{(p)}$ is the group $\mathbb{Z}_{(p)}^{*}$ of units in $\mathbb{Z}_{(p)}$. Consider the map $S^{1} \rightarrow B F_{(p)}$ corresponding to the unit $1-p$ and let $f: \Omega^{2}\left(S^{3}\right) \rightarrow B F_{(p)}$ be the extension to a 2 -fold loop map. It is an observation of Hopkins that the associated 
$p$-local Thom spectrum $T(f)$ is a model of $H \mathbb{Z} / p$ [28]. The argument is similar to that for $H \mathbb{Z} / 2[27 ; 14]$, using that the Thom space of the map $S^{1} \rightarrow B F_{(p)}(1)$ is a mod $p$ Moore space.

Proof of Theorem 1.3 Applying the $p$-local version of Theorem 2 to the 2-fold loop map $f: \Omega^{2}\left(S^{3}\right) \rightarrow B F_{(p)}$, we get that

$$
\operatorname{THH}(H \mathbb{Z} / p) \simeq \operatorname{THH}(T(f)) \simeq H \mathbb{Z} / p \wedge T(\eta \circ B f)
$$

and it follows from the $H \mathbb{Z} / p$ Thom isomorphism theorem that there is a stable equivalence

$$
H \mathbb{Z} / p \wedge T(\eta \circ B f) \simeq H \mathbb{Z} / p \wedge \Omega\left(S^{3}\right)_{+} .
$$

This is (the $p$-local version of) the homotopy theoretical interpretation of the Thom isomorphism [29; 35]. Indeed, given a map $g: X \rightarrow B F_{(p)}$, the condition for $T(g)$ to be $H \mathbb{Z} / p$ orientable is that the composite map

$$
X \stackrel{g}{\rightarrow} B F_{(p)} \longrightarrow B \mathbb{Z}_{(p)}^{*} \longrightarrow B(\mathbb{Z} / p)^{*}
$$

be null homotopic. In our case this holds since $\Omega\left(S^{3}\right)$ is simply connected.

In order to realize $H \mathbb{Z}_{(p)}$ as a Thom spectrum we compose with the canonical map $\Omega^{2}\left(S^{3}\langle 3\rangle\right) \rightarrow \Omega^{2}\left(S^{3}\right)$ as explained in the introduction. The argument for $p$ odd is again similar to that given for $p=2$ in [27; 14].

Theorem 3.8 There is a stable equivalence

$$
\operatorname{THH}\left(H \mathbb{Z}_{(p)}\right) \simeq H \mathbb{Z}_{(p)} \wedge \Omega\left(S^{3}\langle 3\rangle\right)_{+} .
$$

Proof Let $f: \Omega^{2}\left(S^{3}\langle 3\rangle\right) \rightarrow B F_{(p)}$ be the composite map. By the $p$-local version of Theorem 2 we have the stable equivalence

$$
\operatorname{THH}\left(H \mathbb{Z}_{(p)}\right) \simeq \operatorname{THH}(T(f)) \simeq H \mathbb{Z}_{(p)} \wedge T(\eta \circ B f)
$$

and since $\Omega\left(S^{3}\langle 3\rangle\right)$ is simply connected,

$$
H \mathbb{Z}_{(p)} \wedge T(\eta \circ B f) \simeq H \mathbb{Z}_{(p)} \wedge \Omega\left(S^{3}\langle 3\rangle\right)_{+}
$$

by the $H \mathbb{Z}_{(p)}$ Thom isomorphism.

Using that topological Hochschild homology commutes with localization and that $\mathrm{THH}(H \mathbb{Z})$ is a generalized Eilenberg-Mac Lane spectrum, this immediately implies the calculation in Theorem 1.4. 


\subsection{The Thom spectra $M \mathrm{Br}, M \Sigma$ and $M G L(\mathbb{Z})$}

As explained in the introduction, a compatible system of group homomorphisms $G_{n} \rightarrow \mathrm{GL}_{n}(\mathbb{R})$ gives rise to a Thom spectrum $M G$. In particular, we have the systems of groups

$$
\mathrm{Br}_{n} \longrightarrow \Sigma_{n} \longrightarrow \mathrm{GL}_{n}(\mathbb{Z}) \longrightarrow \mathrm{GL}_{n}(\mathbb{R})
$$

given by the braid groups $\mathrm{Br}_{n}$, the symmetric groups $\Sigma_{n}$, and the general linear groups $\mathrm{GL}_{n}(\mathbb{Z})$. Each one of these families has a natural "block sum" pairing, inducing a multiplicative structure on the associated Thom spectrum; see Bullett [12] and Cohen [13]. Indeed, except for $M \mathrm{Br}$, these spectra are in fact commutative symmetric ring spectra [42]. In order to fit these examples in our framework we observe that in each case the induced map $B G_{\infty} \rightarrow B G L(\mathbb{R})$ from the stabilized group factors over Quillen's plus-construction to give a loop map $B G_{\infty}^{+} \rightarrow B \mathrm{GL}(\mathbb{R})$. Furthermore, it follows from the fact that $B G_{\infty} \rightarrow B G_{\infty}^{+}$induces an isomorphism in homology that $M G$ is equivalent to the corresponding Thom spectrum $T\left(B G_{\infty}^{+}\right)$. For the braid groups, Cohen proves [13] that $B \mathrm{Br}_{\infty}^{+}$is equivalent to $\Omega^{2}\left(S^{3}\right)$ and combining this with Mahowald's theorem it follows that $M \mathrm{Br}$ is a model of $H \mathbb{Z} / 2$. Thus, in this case we recover the calculation in Theorem 1.3 for $p=2$. Notice, that the identification of $M \mathrm{Br}$ with $H \mathbb{Z} / 2$ implies that $M \Sigma, M \mathrm{GL}(\mathbb{Z})$ and $M O$ are $H \mathbb{Z} / 2$-module spectra, hence generalized Eilenberg-Mac Lane spectra.

Proof of Theorem 1.5 By the above remarks, $M \Sigma$ is equivalent to the Thom spectrum of the map $B \Sigma_{\infty}^{+} \rightarrow B G L(\mathbb{R})$. It follows from the Barratt-Priddy-Quillen Theorem that $B \Sigma_{\infty}^{+}$is equivalent to the base point component of $Q\left(S^{0}\right)$ and that the map in question is the restriction to the base point component of the infinite loop map $Q\left(S^{0}\right) \rightarrow B \mathrm{GL}(\mathbb{R}) \times \mathbb{Z}$. Hence $\widetilde{Q}\left(S^{1}\right)$ is a delooping of $B \Sigma_{\infty}^{+}$and the result follows from Theorem 3.

Proof of Theorem 1.6 It again follows from the above discussion that $M G L(\mathbb{Z})$ is equivalent to the Thom spectrum of the infinite loop map $B \mathrm{GL}_{\infty}^{+} \rightarrow B \mathrm{GL}(\mathbb{R})$. Here the domain is the base point component of Quillen's algebraic $K$-theory space of $\mathbb{Z}$ and the result follows from Theorem 3.

\section{Operadic products in the category of spaces}

In this section, we adapt the construction of the "operadic" smash product of spectra from [16] to the context of topological spaces. The conceptual foundation of the approach to structured ring spectra undertaken in [16] is the observation that by 
exploiting special properties of the linear isometries operad, it is possible to define a weak symmetric monoidal product on a certain category of spectra such that the (commutative) monoids are precisely the $\left(E_{\infty}\right) A_{\infty}$ ring spectra. Since many of the good properties of this product are a consequence of the nature of the operad, such a construction can be carried out in other categories - for instance, Kriz and May [22] studied an algebraic version of this definition. Following an observation of Mandell, we introduce a version of this construction in the category of spaces. Much of this work appeared in the first author's University of Chicago thesis [6].

\subsection{The weak symmetric monoidal category of $\mathcal{L}(1)$-spaces}

Fix a countably infinite-dimensional real inner product space $\mathbb{U}$, and let $\mathcal{L}(n)$ denote the $n$-th space of the linear isometries operad associated to $\mathbb{U}$; recall that this is the space of linear isometries $\mathcal{L}\left(\mathbb{U}^{n}, \mathbb{U}\right)$. Since $\mathcal{L}\left(\mathbb{U}^{n}, \mathbb{U}\right)$ is contractible and has a free $\Sigma_{n}$ action, the linear isometries operad is an $E_{\infty}$ operad.

Observe that the space $\mathcal{L}(1)=\mathcal{L}(\mathbb{U}, \mathbb{U})$ is a topological monoid. We begin by considering the category of $\mathcal{L}(1)$-spaces: unbased spaces equipped with a map $\mathcal{L}(1) \times X \rightarrow X$ which is associative and unital. We can equivalently regard this category as the category $\mathcal{U}[\mathbb{L}]$ of algebras over the monad $\mathbb{L}$ on the category $\mathcal{U}$ of unbased spaces which takes $X$ to $\mathcal{L}(1) \times X$.

The category $\mathcal{U}[\mathbb{L}]$ admits a product $X \otimes_{\mathcal{L}} Y$ defined in analogy with the product $\wedge_{\mathcal{L}}$ on the category of $\mathbb{L}$-spectra [16, Section I.5.1]. Specifically, there is an action of $\mathcal{L}(1) \times \mathcal{L}(1)$ on $\mathcal{L}(2)$ via the operad structure map $\mathcal{L}(2) \times \mathcal{L}(1) \times \mathcal{L}(1) \rightarrow \mathcal{L}(2)$. In addition, there is a natural action of $\mathcal{L}(1) \times \mathcal{L}(1)$ on $X \times Y$ given by the isomorphism

$$
(\mathcal{L}(1) \times \mathcal{L}(1)) \times(X \times Y) \cong(\mathcal{L}(1) \times X) \times(\mathcal{L}(1) \times Y) .
$$

We define $\otimes_{\mathcal{L}}$ as the balanced product of these two actions.

Definition 4.1 The product $X \otimes_{\mathcal{L}} Y$ is the coequalizer of the diagram

$$
\mathcal{L}(2) \times \mathcal{L}(1) \times \mathcal{L}(1) \times X \times Y \Longrightarrow \mathcal{L}(2) \times X \times Y .
$$

The coequalizer is itself an $\mathcal{L}(1)$-space via the left action of $\mathcal{L}(1)$ on $\mathcal{L}(2)$ by postcomposition.

The arguments of [16, Section I.5] now yield the following proposition.

Proposition 4.2 (i) The operation $\nabla_{\mathcal{L}}$ is associative. For any $j$-tuple $M_{1}, \ldots, M_{k}$ of $\mathcal{L}(1)$-spaces there is a canonical and natural isomorphism of $\mathcal{L}(1)$-spaces

$$
M_{1} \otimes_{\mathcal{L}} \cdots \otimes_{\mathcal{L}} M_{k} \cong \mathcal{L}(k) \times_{\mathcal{L}(1)^{k}} M_{1} \times \cdots \times M_{k}
$$

where the iterated product on the left is associated in any fashion. 
(ii) The operation $\nabla_{\mathcal{L}}$ is commutative. There is a natural isomorphism of $\mathcal{L}(1)-$ spaces

$$
\tau: X \otimes_{\mathcal{L}} Y \cong Y \otimes_{\mathcal{L}} X
$$

with the property that $\tau^{2}=\mathrm{id}$.

There is a corresponding mapping $\mathcal{L}(1)$-space $F_{\bigotimes_{\mathcal{L}}}(X, Y)$; the definition follows [16, Section I.7], and analogously is forced by the desired adjunction.

Proposition 4.3 Let $X, Y$, and $Z$ be $\mathcal{L}(1)$-spaces. Then there is an adjunction homeomorphism

$$
\operatorname{Map}_{\mathcal{U}[\mathbb{L}]}\left(X \otimes_{\mathcal{L}} Y, Z\right) \cong \operatorname{Map}_{\mathcal{U}[\mathbb{L}]}\left(X, F_{\otimes_{\mathcal{L}}}(Y, Z)\right) .
$$

The natural choice for the unit of the product $\nabla_{\mathcal{L}}$ is the point $*$, endowed with the trivial $\mathcal{L}(1)$-action. As in [16, Section 1.8.3], there is a unit map $* \nabla_{\mathcal{L}} X \rightarrow X$ which is compatible with the associativity and commutativity properties of $\otimes_{\mathcal{L}}$.

Proposition 4.4 Let $X$ and $Y$ be $\mathcal{L}(1)$-spaces. There is a natural unit map of $\mathcal{L}(1)-$ spaces $\lambda: * \nabla_{\mathcal{L}} X \rightarrow X$. The symmetrically defined map $X \otimes_{\mathcal{L}} * \rightarrow X$ coincides with the composite $\lambda \tau$. Under the associativity isomorphism $\lambda \tau \otimes_{\mathcal{L}} \mathrm{id} \cong \mathrm{id} \otimes_{\mathcal{L}} \lambda$, and, under the commutativity isomorphism, these maps also agree with $* \otimes_{\mathcal{L}}\left(X \otimes_{\mathcal{L}} Y\right) \rightarrow X \otimes_{\mathcal{L}} Y$.

However, just as is the case in the category of $\mathbb{L}$-spectra, $*$ is not a strict unit for $\nabla_{\mathcal{L}}$ : the unit map $* \nabla_{\mathcal{L}} X \rightarrow X$ is not necessarily an isomorphism. However, it is always a weak equivalence. We omit the proof of this fact, as it is very technical and essentially similar to the proof of the analogous fact for $\mathbb{L}$-spectra $[16,1.8 .5]$. We remark only that it is a consequence of the remarkable point-set properties of the linear isometries operad and the isomorphism $* \nabla_{\mathcal{L}} * \rightarrow *$.

Proposition 4.5 For any $\mathcal{L}(1)-$ space $X$, the unit map $\lambda: * \otimes_{\mathcal{L}} X \rightarrow X$ is a weak equivalence of $\mathcal{L}(1)$-spaces.

In summary, the category $\mathcal{U}[\mathbb{L}]$ is a closed weak symmetric monoidal category, with product $\nabla_{\mathcal{L}}$ and weak unit $*$. Recall that this means that the $\mathcal{U}[\mathbb{L}], \otimes_{\mathcal{L}}, F_{\bigotimes_{\mathcal{L}}}(-,-)$, and $*$ satisfy all of the axioms of a closed symmetric monoidal category except that the unit map is not required to be an isomorphism [16, Section II.7.1]. 


\subsection{Monoids and commutative monoids for $\boldsymbol{\nabla}_{\mathcal{L}}$}

In this section, we study $\otimes_{\mathcal{L}}$-monoids and commutative $\otimes_{\mathcal{L}}$-monoids in $\mathcal{U}[\mathbb{L}]$; these are defined as algebras over certain monads, following [16, Section 2.7]. We show that $\nabla_{\mathcal{L}}$-monoids are $A_{\infty}$ spaces and commutative $\nabla_{\mathcal{L}}$-monoids are $E_{\infty}$ spaces. One can prove this directly, as is done in the algebraic setting in [22, Section V.3.1], but we prefer to follow the categorical approach given for $\mathbb{L}$-spectra and $\wedge_{\mathcal{L}}$ in $[16$, Section II.4].

In any closed weak symmetric monoidal category which is cocomplete, monoids and commutative monoids can be regarded as algebras over certain monads $\mathbb{T}$ and $\mathbb{P}$; in the case of $\mathcal{L}(1)$-spaces, these are defined as follows. Let $X^{\otimes_{j}}$ denote the $j$-fold power with respect to $\nabla_{\mathcal{L}}$, where $X^{0}=*$. Then we have monads on the category of $\mathcal{L}(1)$-spaces given by the formulas

$$
\mathbb{T} X=\bigsqcup_{j \geq 0} X^{\otimes j}, \quad \mathbb{P} X=\bigsqcup_{j \geq 0} X^{\otimes j} / \Sigma_{j},
$$

where the unit is given by the inclusion of $X$ into the coproduct and the product is induced by the obvious identifications (and the unit map, if any indices are 0 ).

On the other hand, we can regard $A_{\infty}$ and $E_{\infty}$ spaces as algebras over monads $\mathbb{B}$ and $\mathbb{C}$ on unbased spaces. Recall that these monads are defined as

$$
\mathbb{B} Y=\bigsqcup_{j \geq 0} \mathcal{L}(j) \times X^{j}, \quad \mathbb{C} Y=\bigsqcup_{j \geq 0} \mathcal{L}(j) \times_{\Sigma_{j}} X^{j},
$$

where here $X^{n}$ indicates the iterated cartesian product. Notice that these monads can be obtained by composing the customary monads on based spaces which define $A_{\infty}$ and $E_{\infty}$ spaces with the monad that adjoins a disjoint basepoint.

The main tool for comparing these various categories of algebras is the lemma [16, Section II.6.1] (or rather its corrected form [17]), which we write out below for clarity.

Lemma 4.6 Let $\mathbb{S}$ be a monad in a category $\mathcal{C}$ and let $\mathbb{R}$ be a monad in the category $\mathcal{C}[\mathbb{S}]$ of $\mathbb{S}$-algebras. If $\mathbb{R}$ preserves reflexive coequalizers in $\mathcal{C}[\mathbb{S}]$, then the category $\mathcal{C}[\mathbb{S}][\mathbb{R}]$ of $\mathbb{R}$-algebras in $\mathcal{C}[\mathbb{S}]$ is isomorphic to the category $\mathcal{C}[\mathbb{R S}]$ of algebras over the composite monad $\mathbb{R} \mathbb{S}$ in $\mathcal{C}$. Moreover, the unit of $\mathbb{R}$ defines a map $\mathbb{S} \rightarrow \mathbb{R}$ of monads in $\mathcal{C}$. An analogous assertion holds for comonads.

Since the relevant monads satisfy the hypotheses of this lemma, we now obtain the following comparison result.

Proposition 4.7 There is an isomorphism of categories between $\nabla_{\mathcal{L}}$-monoids and $A_{\infty}$ spaces structured by the non- $\Sigma$ linear isometries operad. Similarly, there is an isomorphism of categories between commutative $\nabla_{\mathcal{L}}$-monoids and $E_{\infty}$ spaces structured by the linear isometries operad. 
Proof For concreteness, we work with the associative case; the proof is analogous in the commutative situation. The composite $\mathbb{T} \mathbb{L}$ is a monad on unbased spaces. Furthermore, there is an identification of monads on unbased spaces $\mathbb{B} \cong \mathbb{T} \mathbb{L}$; Proposition 4.2 implies an isomorphism of objects, and the comparison of monad structures is immediate. Lemma 4.6 then implies that $\mathbb{B}$-algebras in unbased spaces are equivalent to $\mathbb{T}$-algebras in $\mathcal{L}(1)$-spaces.

\subsection{The symmetric monoidal category of $*$-modules}

In this section we define a subcategory of $\mathcal{L}(1)$-spaces which forms a closed symmetric monoidal category with respect to $\bigotimes_{\mathcal{L}}$. This is necessary for our application to topological Hochschild homology - in order to define the cyclic bar construction as a strict simplicial object, we need a unital product to define the degeneracies. In fact, there are two possible approaches to constructing a symmetric monoidal category from the weak symmetric monoidal category of $\mathcal{L}(1)$-spaces, which parallel the approaches developed in [22] and [16]. If we restrict attention to the category $\mathcal{T}[\mathbb{L}]$ of based $\mathcal{L}(1)$-spaces (where the $\mathcal{L}(1)$ action is trivial on the basepoint), there is a unital product $\star_{\mathcal{L}}$ formed as the pushout

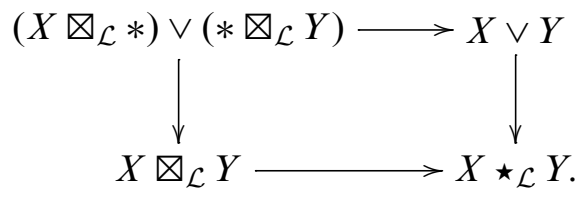

In the algebraic setting of [22], this kind of construction is our only option. However, since there is an isomorphism $* \nabla_{\mathcal{L}} * \cong *$ we can also pursue the strategy of considering a subcategory of $\mathcal{L}(1)$-spaces analogous to the category of $S$-modules [16]. Specifically, observe that the $\mathcal{L}(1)$-space $* \otimes_{\mathcal{L}} X$ is unital in the sense that the unit map $* \nabla_{\mathcal{L}}\left(* \nabla_{\mathcal{L}} X\right) \rightarrow\left(* \nabla_{\mathcal{L}} X\right)$ is an isomorphism.

Definition 4.8 The category $\mathcal{M}_{*}$ of $*$-modules is the subcategory of $\mathcal{L}(1)$-spaces such that the unit map $\lambda: * \nabla_{\mathcal{L}} X \rightarrow X$ is an isomorphism. For $*$-modules $X$ and $Y$, define $X \otimes Y$ as $X \otimes_{\mathcal{L}} Y$ and $F_{\bigotimes}(X, Y)$ as $* \otimes_{\mathcal{L}} F_{\bigotimes_{\mathcal{L}}}(X, Y)$.

The work of the previous section implies that $\mathcal{M}_{*}$ is a closed symmetric monoidal category. For use in establishing a model structure on $\mathcal{M}_{*}$ in Theorem 4.16, we review a more obscure aspect of this category, following the analogous treatment for the category of $S$-modules [16, Section II.2]. The functor $* \otimes_{\mathcal{L}}-$ is not a monad in $\mathcal{L}(1)$-spaces. However, the category of $*$-modules has a "mirror image" category to which it is naturally equivalent, and this equivalence facilitates formal analysis of the category of $*$-modules. 
Let $\mathcal{M}^{*}$ be the full subcategory of counital $\mathcal{L}(1)$-spaces: $\mathcal{L}(1)$-spaces $Z$ such that the counit map $Z \rightarrow F_{\bigotimes_{\mathcal{L}}}(*, Z)$ is an isomorphism. Following the notation of [16, Section II.2], let $f$ denote the functor $F_{\bigotimes_{\mathcal{L}}}(*,-)$ and $s$ denote the functor $* \nabla_{\mathcal{L}}(-)$. Let $r$ be the inclusion of the counital $\mathcal{L}(1)$-spaces into the category of $\mathcal{L}(1)$-spaces, and $\ell$ the inclusion of the unital $\mathcal{L}(1)$-spaces ( $*$-modules) into $\mathcal{L}(1)$-spaces. We have the following easy lemma about these functors.

Lemma 4.9 The functor $f$ is right adjoint to the functor $s$ and left adjoint to the inclusion $r$.

Now, we obtain a pair of mirrored adjunctions

$$
\mathcal{U}[\mathbb{L}] \underset{r f \ell}{\stackrel{s}{\rightleftarrows}} \mathcal{M}_{*} \underset{s}{\stackrel{\ell}{\rightleftarrows}} \mathcal{U}[\mathbb{L}], \quad \mathcal{U}[\mathbb{L}] \underset{r}{\stackrel{f}{\rightleftarrows}} \mathcal{M}^{*} \underset{f}{\stackrel{\ell s r}{\rightleftarrows}} \mathcal{U}[\mathbb{L}] .
$$

The composite of the first two left adjoints is $* \nabla_{\mathcal{L}}(-)$ and the composite of the second two right adjoints is $F_{\square_{\mathcal{L}}}(*,-)$. These are themselves adjoints, and now by the uniqueness of adjoints we have the following consequence.

Lemma 4.10 For an $\mathcal{L}(1)$-space $X$, the maps

$$
* \otimes_{\mathcal{L}} X \longrightarrow * \otimes_{\mathcal{L}} F_{\otimes_{\mathcal{L}}}(*, X)
$$

and

$$
F_{\bigotimes_{\mathcal{L}}}\left(*, * \otimes_{\mathcal{L}} X\right) \longrightarrow F_{\bigotimes_{\mathcal{L}}}(*, X)
$$

are natural isomorphisms.

An immediate consequence of this is that the category $\mathcal{M}_{*}$ and $\mathcal{M}^{*}$ are equivalent, and in particular we see that the category of $*$-modules is equivalent to the category of algebras over the monad $r f$ determined by the adjunction (see the proof of [16, Section II.2.7] for further details).

\subsection{Monoids and commutative monoids in $\mathcal{M}_{*}$}

The monads $\mathbb{T}$ and $\mathbb{P}$ on $\mathcal{L}(1)$-spaces restrict to define monads on $\mathcal{M}_{*}$. The algebras over these monads are monoids and commutative monoids for $\otimes$, respectively. Thus, a $\nabla$-monoid in $\mathcal{M}_{*}$ is a $\nabla_{\mathcal{L}}$-monoid in $\mathcal{L}(1)$ which is also a $*$-module (and similarly for commutative $\otimes$-monoids). The functor $* \nabla_{\mathcal{L}}(-)$ gives us a means to functorially replace $\nabla_{\mathcal{L}}$-monoids and commutative $\otimes_{\mathcal{L}}$-monoids with $\nabla$-monoids and commutative $\otimes$-monoids. 
Proposition 4.11 Given a $\nabla_{\mathcal{L}}$-monoid $X$, the object $* \nabla_{\mathcal{L}} X$ is a $\nabla$-monoid and the map $\lambda: * \nabla_{\mathcal{L}} X \rightarrow X$ is a weak equivalence of $\otimes_{\mathcal{L}}$-monoids. The analogous results in the commutative case hold.

Furthermore, note that the standard formal arguments imply that $\nabla$ is the coproduct in the category $\mathcal{M}_{*}[\mathbb{P}]$.

\subsection{Functors to spaces}

In this section, we discuss two functors which allow us to compare the categories $\mathcal{U}[\mathbb{L}]$ and $\mathcal{M}_{*}$ to spaces. There is a continuous forgetful functor $U: \mathcal{U}[\mathbb{L}] \rightarrow \mathcal{U}$ such that $U(*)=*$. This functor restricts to a continuous forgetful functor $U: \mathcal{M}_{*} \rightarrow U$. In addition, we have another continuous functor from $\mathcal{L}(1)$-spaces to $\mathcal{U}$. There is a map of topological monoids $\theta: \mathcal{L}(1) \rightarrow *$. Associated to any map of monoids is an adjoint pair $\left(\theta_{*}, \theta^{*}\right)$. The right adjoint $\theta^{*}: \mathcal{U} \rightarrow \mathcal{U}[\mathbb{L}]$ is the functor which assigns a trivial action to a space, and the left adjoint is described in the next definition.

Definition 4.12 The monoid map $\mathcal{L}(1) \rightarrow *$ induces a functor $Q: \mathcal{U}[\mathbb{L}] \rightarrow \mathcal{U}$ which takes an $\mathcal{L}(1)$-space $X$ to $* \times_{\mathcal{L}(1)} X . Q$ is the left adjoint to the pullback functor which gives a space $Y$ the trivial $\mathcal{L}(1)$-action. $Q$ restricts to a functor $Q: \mathcal{M}_{*} \rightarrow \mathcal{U}$.

The interest of this second functor $Q$ is that it is strong symmetric monoidal: it allows us to relate $\nabla$ to the cartesian product of spaces.

Lemma 4.13 The functor $Q: \mathcal{U}[\mathbb{L}] \rightarrow \mathcal{U}$ is strong symmetric monoidal with respect to the symmetric monoidal structures induced by $\otimes_{\mathcal{L}}$ and $\times$ respectively. Correspondingly, the functor $Q: \mathcal{M}_{*} \rightarrow \mathcal{U}$ is strong symmetric monoidal with respect to $\nabla$ and $\times$ respectively.

Proof Let $X$ and $Y$ be $\mathcal{L}(1)$-spaces. We need to compare $* \times_{\mathcal{L}(1)}\left(X \nabla_{\mathcal{L}} Y\right)$ and $\left(* \times_{\mathcal{L}(1)} X\right) \times\left(* \times_{\mathcal{L}(1)} Y\right)$. Observe that $\mathcal{L}(2)$ is homeomorphic to $\mathcal{L}(1)$ as a left $\mathcal{L}(1)$-space, by composing with an isomorphism $g: \mathbb{U}^{2} \rightarrow \mathbb{U}$. Therefore we have isomorphisms

$$
\begin{aligned}
* \times_{\mathcal{L}(1)}\left(X \otimes_{\mathcal{L}} Y\right) & =* \times_{\mathcal{L}(1)} \mathcal{L}(2) \times_{\mathcal{L}(1) \times \mathcal{L}(1)}(X \times Y) \\
& \cong\left(* \times_{\mathcal{L}(1)} X\right) \times\left(* \times_{\mathcal{L}(1)} Y\right)
\end{aligned}
$$

One checks that the required coherence diagrams commute. The result now follows, as $* \times_{\mathcal{L}(1)} * \cong *$.

The preceding result implies that $Q$ takes $\otimes_{\mathcal{L}}$-monoids and $\otimes$-monoids to topological monoids. In Section 4.7, we will describe conditions under which the natural map $U X \rightarrow Q X$ is a weak equivalence. 


\subsection{Model category structures}

In this section, we describe the homotopy theory of the categories described in the previous sections. We begin by establishing model category structures on the various categories and identifying the cofibrant objects. We rely on the following standard lifting result (eg [43, A.3]).

Theorem 4.14 Let $\mathcal{C}$ be a cofibrantly generated model category where all objects are fibrant, with generating cofibrations $I$ and acyclic generating cofibrations $J$. Assume that the domains of $I$ and $J$ are small relative to the classes of transfinite pushouts of maps in $I$ and $J$ respectively. Let $\mathbb{A}$ be a continuous monad on $\mathcal{C}$ which commutes with filtered direct limits and such that all $\mathbb{A}$-algebras have a path object. Then the category $\mathcal{C}[\mathbb{A}]$ has a cofibrantly generated model structure in which the weak equivalences and fibrations are created by the forgetful functor to $\mathcal{C}$. The generating cofibrations and acyclic cofibrations are the sets $\mathbb{A} I$ and $\mathbb{A} J$ respectively.

Furthermore, when $\mathcal{C}$ is topological, provided that the monad $\mathbb{A}$ preserves reflexive coequalizers, the category $\mathcal{C}[\mathbb{A}]$ will also be topological [16, Section VII.2.10]. All of the monads that arise in this paper preserve reflexive coequalizers [16, Section II.7.2]. We now record the model structures on the categories we study. We assume that $\mathcal{U}$ and $\mathcal{T}$ are equipped with the standard model structure in which the weak equivalences are the weak homotopy equivalences and the fibrations are the Serre fibrations.

Theorem 4.15 The category $\mathcal{U}[\mathbb{L}]$ admits a cofibrantly generated topological model structure in which the fibrations and weak equivalences are detected by the forgetful functor to spaces. Limits and colimits are constructed in the underlying category $\mathcal{U}$.

We use an analogous argument to deduce the existence of a topological model structure on $*$-modules, employing the technique used in the proof of [16, Section VII.4.6]. The point is that counital $\mathcal{L}(1)$-spaces are algebras over a monad, and moreover there is an equivalence of categories between counital $\mathcal{L}(1)$-spaces and unital $\mathcal{L}(1)$-spaces; recall the discussion of the "mirror image" categories above. The following theorem then follows once again from Theorem 4.14.

Theorem 4.16 The category $\mathcal{M}_{*}$ admits a cofibrantly generated topological model structure. A map $f: X \rightarrow Y$ of $*$-modules is

- a weak equivalence if the map $U f: U X \rightarrow U Y$ of spaces is a weak equivalence,

- a fibration if the induced map $F_{\square}(*, X) \rightarrow F_{\square}(*, Y)$ is a fibration of spaces.

Colimits are created in the category $\mathcal{U}[\mathbb{L}]$, and limits are created by applying $* \otimes(-)$ to the limit in the category $\mathcal{U}[\mathbb{L}]$. 
Notice that although the fibrations have changed (since the functor to spaces which we're lifting over is $F_{\bigotimes}(*,-)$ and not the forgetful functor), nonetheless this category still has the useful property that all objects are fibrant.

Lemma 4.17 All objects in the category of $*$-modules are fibrant.

Proof This is an immediate consequence of the isomorphism $F_{\square}(*, *) \cong *$ and the fact that all spaces are fibrant.

As a consequence, we obtain the following summary theorem about model structures on monoids and commutative monoids.

Theorem 4.18 The categories $(\mathcal{U}[\mathbb{L}])[\mathbb{T}]$ and $(\mathcal{U}[\mathbb{L}])[\mathbb{P}]$ of $\otimes_{\mathcal{L}}$-monoids and commutative $\nabla_{\mathcal{L}}$-monoids in $\mathcal{L}(1)$-spaces admit cofibrantly generated topological model structures in which the weak equivalences and fibrations are maps which are weak equivalences and fibrations of $\mathcal{L}(1)$-spaces. Similarly, the categories $\mathcal{M}_{*}[\mathbb{T}]$ and $\mathcal{M}_{*}[\mathbb{P}]$ of $\otimes$-monoids and commutative $\otimes$-monoids in $\mathcal{M}_{*}$ admit cofibrantly generated topological model structures in which the weak equivalences and fibrations are the maps which are weak equivalences and fibrations in $\mathcal{M}_{*}$. Limits are created in the underlying category and colimits are created as a certain coequalizer [16, Section II.7.4].

Next, in order to work with left derived functors associated to functors with domain one of these categories, we describe a convenient characterization of the cofibrant objects. Let $\mathcal{C}$ be a cofibrantly generated model category with generating cofibrations $\left\{A_{i} \rightarrow B_{i}\right\}$ such that $A_{i}$ and $B_{i}$ are compact and let $\mathbb{A}$ be a continuous monad satisfying the hypotheses of Theorem 4.14. A cellular object in the category $\mathcal{C}[\mathbb{A}]$ is a (countable) sequential colimit $X=\operatorname{colim}_{i} X_{i}$, with $X_{0}=*$ and $X_{i+1}$ defined as the pushout

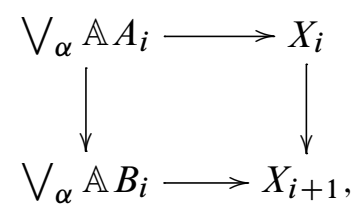

where here $\alpha$ is some indexing subset of the indexing set of the generating cofibrations. The following result is then a formal consequence of the proof of Theorem 4.14 and the compactness of the domains and codomains in the generating cofibrations.

Proposition 4.19 In the model structures of Theorem 4.15, Theorem 4.16 and Theorem 4.18 , the cofibrant objects are retracts of cellular objects. 
Note that this proposition holds more generally with an appropriate transfinite description of the cellular objects in the absence of the compactness conditions.

These categories also satisfy appropriate versions of the "Cofibration Hypothesis" of [16, Section VII]. That is, for a cellular object the maps $X_{n} \rightarrow X_{n+1}$ are (unbased) Hurewicz cofibrations and the sequential colimit $X=\operatorname{colim}_{i} X_{i}$ can be computed in the underlying category of spaces.

\subsection{Homotopical analysis of $\boldsymbol{\bigotimes}_{\mathcal{L}}$}

In this section, we discuss the homotopical behavior of $\nabla_{\mathcal{L}}$. We will show that the left derived functor of $\nabla_{\mathcal{L}}$ is the cartesian product. The analysis begins with an essential proposition based on a useful property of $\mathcal{L}(2)$.

Proposition 4.20 For spaces $X$ and $Y$, there are isomorphisms of $\mathcal{L}(1)$-spaces

$$
(\mathcal{L}(1) \times X) \otimes_{\mathcal{L}}(\mathcal{L}(1) \times Y) \cong \mathcal{L}(2) \times(X \times Y) \cong \mathcal{L}(1) \times(X \times Y) .
$$

As a consequence, if $M$ and $N$ are cell $\mathcal{L}(1)$-spaces then so is $M \nabla_{\mathcal{L}} N$. If $M$ and $N$ are cell $*$-modules, then so is $M \otimes N$.

Proof The first isomorphism is immediate from the definitions. The second is a consequence of the fact that $\mathcal{L}(2)$ is isomorphic to $\mathcal{L}(1)$ as an $\mathcal{L}(1)$-space via choice of a linear isometric isomorphism $f: \mathbb{U}^{2} \rightarrow \mathbb{U}$; there is then a homeomorphism $\gamma: \mathcal{L}(1) \times\{f\} \rightarrow \mathcal{L}(2)$. The last two statements now follow by induction from the analogous result for the cartesian product of cell spaces.

With this in mind, we can prove the following result regarding the homotopical behavior of $\otimes$.

Theorem 4.21 The category $\mathcal{M}_{*}$ is a monoidal model category with respect to the symmetric monoidal product $\otimes$ and unit $*$.

Proof We need to verify that $\mathcal{M}_{*}$ satisfies the pushout-product axiom [43, 2.1]. Thus, for cofibrations $A \rightarrow B$ and $X \rightarrow Y$, we must show that the map

$$
(A \otimes Y) \coprod_{A \otimes X}(B \otimes X) \longrightarrow B \otimes Y
$$

is a cofibration, and a weak equivalence if either $A \rightarrow B$ or $X \rightarrow Y$ was. Since $\mathcal{M}_{*}$ is cofibrantly generated, it suffices to check this on generating (acyclic) cofibrations. Therefore, we can reduce to considering a pushout-product of the form

$$
P \longrightarrow\left(* \otimes\left(\mathcal{L}(1) \times B^{\prime}\right)\right) \otimes\left(* \otimes\left(\mathcal{L}(1) \times Y^{\prime}\right)\right),
$$


where $P$ is the pushout

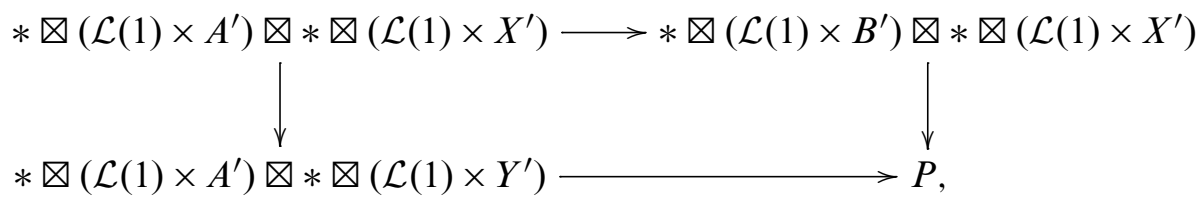

for $A^{\prime} \rightarrow B^{\prime}$ and $X^{\prime} \rightarrow Y^{\prime}$ generating cofibrations in $\mathcal{U}$. Using the fact that $(* \otimes M) \otimes$ $(* \otimes N) \cong * \otimes(M \otimes N)$ and the fact that $* \otimes(-)$ is a left adjoint, we can bring the $* \otimes(-)$ outside. Similarly, using Proposition 4.20 we can bring $\mathcal{L}(1) \times(-)$ outside and rewrite as

$$
* \otimes\left(\mathcal{L}(1) \times\left(A^{\prime} \times Y^{\prime} \coprod_{A^{\prime} \times X^{\prime}} B^{\prime} \times X^{\prime} \longrightarrow B^{\prime} \times Y^{\prime}\right)\right) .
$$

Finally, the pushout-product axiom for $\mathcal{U}$ implies that it suffices to show that $* \otimes$ $(\mathcal{L}(1) \times-)$ preserves cofibrations and weak equivalences. By construction of the model structures, it follows that $\mathcal{L}(1) \times(-)$ and $* \otimes(-)$ preserve cofibrations. Furthermore, $\mathcal{L}(1) \times(-)$ evidently preserves weak equivalences, and the analogous result for $* \otimes(-)$ follows from the fact that the unit map $\lambda$ is a weak equivalence.

The previous theorem implies that for a cofibrant $*$-module $X$, the functor $X \otimes(-)$ is a Quillen left adjoint. In particular, we can compute the derived $\otimes$ product by working with cofibrant objects in $\mathcal{M}_{*}$. Having established the existence of the derived $\triangle$ product, we now will compare it to the cartesian product of spaces.

The analogues in this setting of [16, Section I.4.6] and [16, Section II.1.9] yield the following helpful lemma.

Lemma 4.22 If $X$ is a cofibrant $\mathcal{L}(1)$-space, then $X$ is homotopy equivalent as an $\mathcal{L}(1)$-space to a free $\mathcal{L}(1)$-space $\mathcal{L}(1) \times X^{\prime}$, where $X^{\prime}$ is a cofibrant space. If $Z$ is a cofibrant $*$-module, then $Z$ is homotopy equivalent as a $*$-module to a free $*$-module $* \otimes Z^{\prime}$, where $Z^{\prime}$ is a cofibrant $\mathcal{L}(1)$-space.

We now use this to analyze the behavior of the forgetful functor on $\otimes$. Choosing a linear isometric isomorphism $f: \mathbb{U}^{2} \rightarrow \mathbb{U}$, for $\mathcal{L}(1)$-spaces $X$ and $Y$ there is a natural map $\alpha: U X \times U Y \rightarrow \mathcal{L}(2) \times(X \times Y) \rightarrow X \otimes_{\mathcal{L}} Y$.

Proposition 4.23 Let $X$ and $Y$ be cofibrant $\mathcal{L}(1)$-spaces. Then the natural map $\alpha: U X \times U Y \rightarrow U\left(X \otimes_{\mathcal{L}} Y\right)$ is a weak equivalence of spaces. Let $X$ and $Y$ be cofibrant $*$-modules. Then the natural map $\alpha: U X \times U Y \rightarrow U(X \otimes Y)$ is a weak equivalence of spaces. 
Proof Lemma 4.22 allows us to reduce to the case of free $\mathcal{L}(1)$-spaces and free $*$-modules, and the result then follows from Proposition 4.20.

There is also a map $U\left(X \otimes_{\mathcal{L}} Y\right) \rightarrow U X \times U Y$ induced by the universal property of the product; it follows from Proposition 4.23 that this map is a weak equivalence as well under the hypotheses of the proposition.

The analogous result for commutative $\nabla$-monoids follows from a result of $[4,6.8]$. They prove that for cofibrant $E_{\infty}$ spaces $X$ and $Y$ structured by the linear isometries operad, the natural map $X \bigsqcup Y \rightarrow X \times Y$ from the coproduct to the product is a weak equivalence. Since the coproduct in $\mathcal{M}_{*}[\mathbb{P}]$ is precisely $\otimes$, this implies the natural map $X \otimes Y \rightarrow X \times Y$ is a weak equivalence. To handle the case of associative $\otimes$-monoids, we exploit the following analysis of the underlying $\mathcal{L}(1)$-space of a cell $\otimes$-monoid, following [16, Section VII.6.2].

Proposition 4.24 The underlying $*-$ module associated to a cell associative monoid is cell.

To prove this, we need to briefly recall some facts about simplicial objects in $\mathcal{L}(1)-$ spaces. Recall that for the categories we are studying there are internal and external notions of geometric realization. We need the following compatibility result.

Lemma 4.25 Let $X$ be a simplicial object in any of the categories $\mathcal{U}[\mathbb{L}], \mathcal{M}_{*}$, $(\mathcal{U}[\mathbb{L}])[\mathbb{T}]), \mathcal{M}_{*}[\mathbb{T}],(\mathcal{U}[\mathbb{L}][\mathbb{P}])$ or $\mathcal{M}_{*}[\mathbb{P}]$. Then there is an isomorphism between the internal realization $|X$.$| and the external realization |U X$.$| .$

Proof First, assume that $X$ is a simplicial object in $\mathcal{U}[\mathbb{L}],(\mathcal{U}[\mathbb{L}])[\mathbb{T}]$ or $(\mathcal{U}[\mathbb{L}])[\mathbb{P}]$. In all cases, the argument is essentially the same; we focus on $\mathcal{U}[\mathbb{L}]$. For any simplicial space $Z$., there is an isomorphism of spaces $\mathbb{L}|Z| \cong|\mathbb{L} Z|$. Now, since $* \nabla_{\mathcal{L}} \mid X$. $\mid \cong$ $\mid * \nabla_{\mathcal{L}} X$. , the realization of a $*$-module is a $*$-module. The remaining parts of the lemma follow.

We now begin the proof of Proposition 4.24.

Proof First, observe that we have the following analogue of [16, Section VII.6.1], which holds by essentially the same proof: for $\otimes$-monoids $A$ and $B$ which are cell $*$-modules, the underlying $*$-module of the coproduct in the category of $\nabla$-monoids is a cell $*$-module.

Next, assume that $X$ is a cell $*-$ module. Then $X \otimes X \otimes \cdots \otimes X$ is a cell $*$-module. Since $X \rightarrow C X$ is cellular, the induced map $\mathbb{T} X \rightarrow \mathbb{T} C X$ is the inclusion of a 
subcomplex. Let $Y_{n}$ be a $\otimes$-monoid which is a cell $*$-module and consider the pushout of $\otimes$-monoids

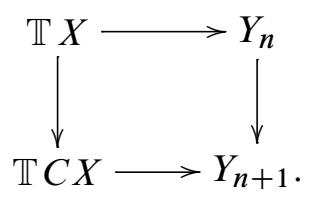

By passage to colimits, it suffices to show that $Y_{n+1}$ is a cell $*$-module. We rely on a description of the pushout of $\nabla$-monoids as the realization of a simplicial $*-$ module. By the argument of [16, Section XII.2.3], we know that $\mathbb{T}$ preserves Hurewicz cofibrations of $*$-modules. The argument of [16, Section VII.3.8] implies that we can describe the pushout $Y_{n+1}$ as the double mapping cylinder $M\left(\mathbb{T} C X, \mathbb{T} X, Y_{n}\right)$, and the argument of [16, Section VII.3.7] establishes that this double mapping cylinder is isomorphic to the realization of the two-sided bar construction with $k$-simplices

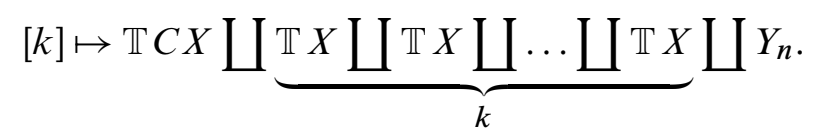

Temporarily assuming that $\mathbb{T} C X$ is a cell $*$-module, since the $k$-simplices of this bar construction are cell $*$-modules and the face and degeneracy maps are cellular, the realization is itself a cell $*$-module. To see that $\mathbb{T} C X$ is a cell $*$-modules, observe that since $\mathbb{T}$ preserves tensors and pushouts, $\mathbb{T} C X \cong * \coprod_{\mathbb{T} X}(\mathbb{T} X \otimes I)$ can be described via an analogous double mapping cylinder and the same argument shows that it is a cell $*$-module.

Finally, we study the functor $Q$. Since we wish to use $Q$ to provide a functorial rectification of associative monoids, we need to determine conditions under which the natural map $U X \rightarrow Q X$ is a weak equivalence. Note that we do not expect this map to be a weak equivalence for commutative monoids, as that would provide a functorial rectification of $E_{\infty}$ spaces to commutative topological monoids.

Proposition 4.26 Let $X$ be a cell $\mathcal{L}(1)$-space. Then the map $U X \rightarrow Q X$ is a weak equivalence of spaces. Let $X$ be a cell $*$-module. Then the map $U X \rightarrow Q X$ is a weak equivalence of spaces. Finally, let $X$ be a cell $\otimes$-monoid in $\mathcal{M}_{*}$. Then the map $U X \rightarrow Q X$ is a weak equivalence of spaces.

Proof First, assume that $X$ is a cell $\mathcal{L}(1)$-space. Since colimits in $\mathcal{L}(1)$-spaces are created in $\mathcal{U}, U$ commutes with colimits. Moreover, since $Q$ is a left adjoint, it also commutes with colimits. By naturality and the fact that the maps $X_{n} \rightarrow X_{n+1}$ in the 
cellular filtration are Hurewicz cofibrations, it suffices to consider the attachment of a single cell. Applying $Q$ to the diagram

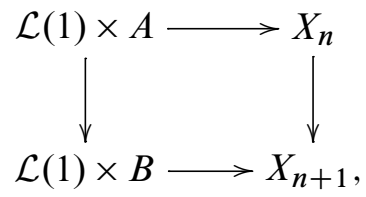

since $Q(\mathcal{L}(1) \times A) \cong A$ we obtain the pushout

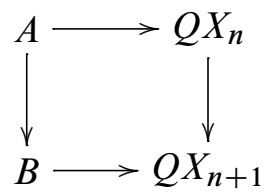

and therefore the result follows by induction, as $Q\left(X_{0}\right)=Q(*)=*$. Next, assume that $X$ is a cell $*$-module. In an analogous fashion, we can reduce to the consideration of the free cells. Since $Q$ is strong symmetric monoidal, $Q\left(* \nabla_{\mathcal{L}}(\mathcal{L}(1) \times Z)\right) \cong$ $* \times Q(\mathcal{L}(1) \times Z)$. Since the unit map $\lambda$ is always a weak equivalence, once again we induct to deduce the result. The case for cell $\otimes$-monoids now follows from Proposition 4.24 .

Therefore, for cell objects in $\mathcal{M}_{*}[\mathbb{T}], Q$ provides a functorial rectification to topological monoids.

\section{Implementing the axioms for $\mathcal{L}(1)$-spaces}

In this section, we will show that the Lewis-May Thom spectrum functor $T$ restricted to the category of $*$-modules over $(* \otimes B G)$ satisfies our axioms. We begin by reviewing the essential properties of the Lewis-May functor.

\subsection{Review of the properties of $T_{\mathscr{S}}$}

As discussed in Section 2.1, the Lewis-May construction of the Thom spectrum yields a functor $T_{\mathscr{S}}: \mathcal{U} / B G \rightarrow \mathscr{Y}$. If we work in the based setting $\mathcal{T} / B G$, we obtain a functor to $\mathscr{S} \backslash S$, spectra under $S$, where for $f: X \rightarrow B G$ the unit $S \rightarrow T_{\mathscr{S}}(f)$ is induced by the inclusion $* \rightarrow X$ over $B G$. In this section, we review various properties of $T_{\mathscr{S}}$ which we will need in verifying the axioms for the version of the Thom spectrum functor we will construct in the context of $\mathcal{L}(1)$-spaces. 
Theorem 5.1 (i) Let $f: * \rightarrow B G$ be the basepoint inclusion. Then $T_{\mathscr{S}}(f) \cong S$.

(ii) [23, 7.4.3] The functor $T_{\mathscr{Y}}: \mathcal{U} / B G \rightarrow \mathscr{Y}$ preserves colimits.

(iii) The functor $T_{\mathscr{S}}: \mathcal{T} / B G \rightarrow \mathscr{S} \backslash S$ preserves colimits.

(iv) $[23,7.4 .6]$ Let $f: X \rightarrow B G$ be a map and $A$ a space. Let $g$ be the composite

$$
X \times A \stackrel{\pi}{\longrightarrow} X \stackrel{f}{\longrightarrow} B G,
$$

where $\pi$ is the projection away from $A$. Then

$$
T_{\mathscr{S}}(g) \cong A_{+} \wedge T_{\mathscr{S}}(f) .
$$

(v) [23, 7.4.9] If $f: X \rightarrow B G$ and $g: X^{\prime} \rightarrow B G$ are $T$-good maps such that there is a weak equivalence $h: X \simeq X^{\prime}$ over $B G$, then there is a stable equivalence $M f \simeq M g$ given by the map of Thom spectra induced by $h$.

(vi) [23, 7.4.10] If $f: X \rightarrow B G$ and $g: X \rightarrow B G$ are $T$-good maps which are homotopic, then there is a stable equivalence $M f \simeq M g$. However, the stable equivalence depends on the homotopy.

Notice that taking $A=I$, item (iv) of the preceding theorem implies that the functor $T_{\mathscr{S}}$ converts fiberwise homotopy equivalences into homotopy equivalences in $\mathscr{S} \backslash S$. Similarly, item (iii) and (iv) imply that $T_{\mathscr{S}}$ preserves Hurewicz cofibrations. The requirement that the maps $X \rightarrow B G$ be $T$-good that appears in the homotopy invariance results suggest that when dealing with spaces over $B F$, a better functor to consider might be the composite $T_{\mathscr{S}} \Gamma$. Unfortunately, the interaction of $\Gamma$ with some of the constructions we are interested in (notably extended powers) is complicated; see Section 6 for further discussion.

Next, we review the multiplicative properties of this version of the Thom spectrum functor. Based spaces with actions by the linear isometries operad $\mathcal{L}$ can be regarded as algebras with respect to an associated monad $\mathbb{C}$ and spectra in $\mathscr{S} \backslash S$ which are $E_{\infty}$-ring spectra structured by the linear isometries operad can be regarded as algebras with respect to an analogous monad we will also denote $\mathbb{C}$. In order to understand the interaction of the Thom spectrum functor with these monads, we need to describe the role of the twisted half-smash product. Given a map $\chi: X \rightarrow \mathcal{L}\left(\mathbb{U}^{j}, \mathbb{U}\right)$ and maps $f_{i}: Y_{i} \rightarrow B G, 1 \leq i \leq j$, we define a map $\chi \times \Pi_{i} f_{i}$ as the composite

$$
X \times \Pi_{i} Y_{i} \stackrel{\Pi_{i} f_{i}}{\longrightarrow} X \times \Pi_{i} B G \stackrel{\chi_{*}}{\longrightarrow} B G
$$

where here $\chi_{*}$ denotes the map induced on $B G$ by $\chi$. Given a subgroup $\pi \subset \Sigma_{n}$ such that $X$ is a $\pi$-space and $\chi$ is a $\pi$-map, then $\chi \times \Pi_{i} f_{i}$ is a $\pi$-map (letting $\pi$ act trivially on $B G)$ and $T_{\mathscr{S}}\left(\chi \times \Pi_{i} f_{i}\right)$ is a spectrum with $\pi$-action. 
The following theorem is $[23,7.6 .1]$.

Theorem 5.2 (i) In the situation above,

$$
T_{\mathscr{S}}\left(\chi \times \Pi_{i} f_{i}\right) \cong X \ltimes\left(\bigwedge_{i} T_{\mathscr{S}}\left(f_{i}\right)\right) .
$$

(ii) Passing to orbits, there is an isomorphism

$$
T_{\mathscr{S}}\left(\chi \times_{\Sigma_{n}} \Pi_{i} f_{i}\right) \cong X \ltimes_{\Sigma_{n}}\left(\bigwedge_{i} T_{\mathscr{S}}\left(f_{i}\right)\right) .
$$

Here $\bar{\wedge}$ denotes the external smash product.

The following corollary is an immediate consequence.

Corollary 5.3 For $f: X \rightarrow B G$ a map of spaces, let $\mathbb{L} f$ denote the composite

$$
\mathcal{L}(1) \times X \longrightarrow \mathcal{L}(1) \times B G \longrightarrow B G,
$$

where the last map is given by the $E_{\infty}$ structure of $B G$ as a space over the linear isometries operad. Then $T_{\mathscr{S}}(\mathbb{L} f) \cong \mathbb{L} T_{\mathscr{S}}(f)$.

Theorem 5.2 is the foundation of the essential technical result that describes the behavior of this Thom spectrum functor in the presence of operadic multiplications. Given a map $f: X \rightarrow B G$, there is an induced monad $\mathbb{C}_{B G}$ on $\mathcal{T} / B G$ specified by defining $\mathbb{C}_{B G} f$ as the composite $\mathbb{C} X \rightarrow \mathbb{C} B G \rightarrow B G$. Lewis proves the following [23, 7.7.1].

Theorem 5.4 Given a map $f: X \rightarrow B F$, there is an isomorphism $\mathbb{C} T_{\mathscr{S}}(f) \cong$ $T_{\mathscr{S}}\left(\mathbb{C}_{B G} f\right)$, and this isomorphism is coherently compatible with the unit and multiplication maps for these monads.

This result has the following corollary; the first part of this is one of the central conclusions of Lewis' thesis, and the second part is a consequence explored at some length in the first author's thesis [6] and forthcoming paper [5].

Theorem 5.5 (i) The functor $T_{\mathscr{S}}$ restricts to a functor

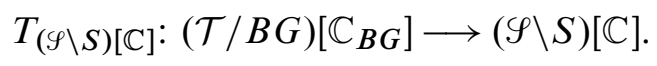

(ii) The functor

$$
T_{(\mathscr{P} \backslash S)[\mathbb{C}]}:(\mathcal{T} / B G)\left[\mathbb{C}_{B G}\right] \longrightarrow \mathscr{S}[\mathbb{C}]
$$

preserves colimits and tensors with unbased spaces. 


\subsection{Verification of the axioms}

We begin by studying the behavior of $T_{\mathscr{S}}$ in the context of $\mathcal{L}(1)$-spaces. The $E_{\infty}$ space $B G$ constructed as the colimit over the inclusions of the $\mathcal{V}$-space $V \mapsto B G(V)$ is a commutative monoid for $\otimes_{\mathcal{L}}$. Therefore, the category $\mathcal{U}[\mathbb{L}] / B G$ has a weak symmetric monoidal product: Given $f: X \rightarrow B G$ and $g: Y \rightarrow B G$, the product $f \nabla_{\mathcal{L}} g$ is defined as

$$
f \otimes_{\mathcal{L}} g: X \otimes_{\mathcal{L}} Y \longrightarrow B G \otimes_{\mathcal{L}} B G \longrightarrow B G .
$$

The unit is given by the trivial map $* \rightarrow B G$. We define

$$
T_{\mathcal{L}}: \mathcal{U}[\mathbb{L}] / B G \longrightarrow \mathscr{S}[\mathbb{L}]
$$

as the Lewis-May Thom spectrum functor $T_{\mathscr{S}}$ restricted to $\mathcal{U}[\mathbb{L}] / B G$ : Corollary 5.3 implies that $T_{\mathscr{S}}$ takes values in $\mathbb{L}$-spectra on $\mathcal{U}[\mathbb{L}] / B G$. Moreover, $T_{\mathcal{L}}$ is strong symmetric monoidal (up to unit).

Proposition 5.6 Given $f: X \rightarrow B G$ and $g: Y \rightarrow B G$, there is a coherently associative isomorphism $T_{\mathcal{L}}(f \otimes g) \cong T_{\mathcal{L}}(f) \wedge_{\mathcal{L}} T_{\mathcal{L}}(g)$.

Proof We can describe $f \otimes g: X \otimes Y \rightarrow B G$ as the natural map to $B G$ associated to the coequalizer describing $X \otimes Y$. Theorem 5.1 implies that $T_{\mathcal{L}}$ commutes with this coequalizer and Theorem 5.2 implies that

$$
T_{\mathscr{S}}(\mathcal{L}(2) \times \mathcal{L}(1) \times \mathcal{L}(1) \times(X \times Y)) \cong(\mathcal{L}(2) \times \mathcal{L}(1) \times \mathcal{L}(1)) \ltimes\left(\left(T_{\mathscr{S}} f\right) \bar{\wedge}\left(T_{\mathscr{S}} g\right)\right)
$$

and

$$
T_{\mathscr{S}}(\mathcal{L}(2) \times(X \times Y)) \cong \mathcal{L}(2) \ltimes\left(\left(T_{\mathscr{S}} f\right) \bar{\wedge}\left(T_{\mathscr{S}} g\right)\right) .
$$

Inspection of the maps then verifies that the resulting coequalizer is precisely the coequalizer defining $T_{\mathcal{L}}(f) \wedge_{\mathcal{L}} T_{\mathcal{L}}(g)$.

Now we restrict to the subcategory $\mathcal{M}_{*}$. For a $₫$-monoid in $\mathcal{M}_{*}$ with multiplication $\mu$, unitality implies that there is a commutative diagram

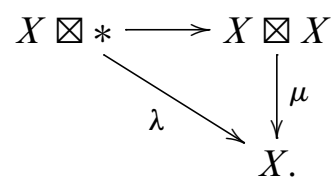

In conjunction with Proposition 5.6, this diagram implies that given a map $f: X \rightarrow B G$, there is an isomorphism

$$
T_{\mathcal{L}}\left(* \otimes_{\mathcal{L}} X \longrightarrow * \otimes_{\mathcal{L}} B G \longrightarrow B G\right) \cong S \wedge_{\mathcal{L}} T_{\mathcal{L}}(f),
$$


which implies that the following definition is sensible. We let $\mathcal{M}_{S}$ denote the category of $S$-modules from [16]; this is our symmetric monoidal category $\mathcal{S}$ of spectra in this setting.

Definition 5.7 The category $\mathcal{A}$ is simply $\mathcal{M}_{*}$. Define $B G_{\mathcal{A}}$ to be $* \nabla_{\mathcal{L}} B G$. We define a Thom spectrum functor

$$
T_{\mathcal{A}}: \mathcal{A} / B G_{\mathcal{A}} \longrightarrow \mathcal{M}_{S}
$$

given $f: X \rightarrow B G_{\mathcal{A}}$ by applying $T_{\mathcal{L}}$ to the composite

$$
X \stackrel{f}{\longrightarrow} B G_{\mathcal{A}} \stackrel{\lambda}{\longrightarrow} B G .
$$

It follows immediately from Proposition 5.6 and the observation preceding the definition that $T_{\mathcal{A}}$ is a strong symmetric monoidal functor from $\mathcal{M}_{*} / B G_{\mathcal{A}}$ to $\mathcal{M}_{S}$. Since Theorem 5.5 implies that $T_{\mathcal{A}}$ commutes with colimits and tensors, $T_{\mathcal{A}}$ commutes with geometric realization and therefore the pair $\mathcal{A}, T_{\mathcal{A}}$ satisfies Axioms (A1) and (A2).

Next, we use Theorem 4.18 to choose a cofibrant replacement functor $c$ on the category $\mathcal{M}_{*}[\mathbb{T}]$ such that for any object $X$ in $\mathcal{A}, c X$ is a cell monoid [19]. Note that cell monoids are well-based; one proves this by an inductive argument using the "Cofibration Hypothesis" (see Ando et al [1,3.22] for details). Then given an object $f: X \rightarrow B G_{\mathcal{A}}$, we define $C f$ to be the composite

$$
c X \stackrel{\simeq}{\longrightarrow} X \longrightarrow B G_{\mathcal{A}} .
$$

We will work with the notion of flatness encapsulated in the definition of the class of $S$-modules $\overline{\mathcal{F}_{S}}$ by Basterra [3, 9.6] (see also Elmendorf et al [16, VII.6.4]). Let $\mathcal{F}_{S}$ denote the collection of modules of the form $S \wedge_{\mathcal{L}} \mathcal{L}(j) \ltimes_{G} K$ where $K$ is a $G$-spectrum (for $G \subset \Sigma_{j}$ ) which has the homotopy type of a $G-\mathrm{CW}$ - spectrum. Then $\overline{\mathcal{F}_{S}}$ is the closure of $\mathcal{F}_{S}$ under finite $\wedge$, wedges, pushouts along cofibrations, colimits of countable sequences of cofibrations, homotopy equivalences, and "stabilization" (in which if $\Sigma M$ is in $\overline{\mathcal{F}_{S}}$ then so is $M$ ). The point of this definition is that for $S$-modules $M$ and $N$ in the class $\overline{\mathcal{F}_{S}}$, the point-set smash product $M \wedge N$ represents the derived smash product [16, VII.6.7; 3, 9.5].

Lemma 5.8 Let $f: X \rightarrow B G_{\mathcal{A}}$ be an object of $\mathcal{M}_{*}[\mathbb{T}] / B G_{\mathcal{A}}$ such that $X$ is a cell monoid. Then the underlying $S$-module of the $S$-algebra $T_{\mathcal{A}}(f)$ is in the class $\overline{\mathcal{F}_{S}}$.

Proof We proceed by induction. Since the class $\overline{\mathcal{F}_{S}}$ is closed under colimits of countable sequences of cofibrations and $T_{\mathcal{A}}$ commutes with colimits and preserves 
Hurewicz cofibrations, it suffices to consider the case in which $X$ is generated by the attachment of finitely many cells. Thus, we can reduce to consideration of a pushout square of the form

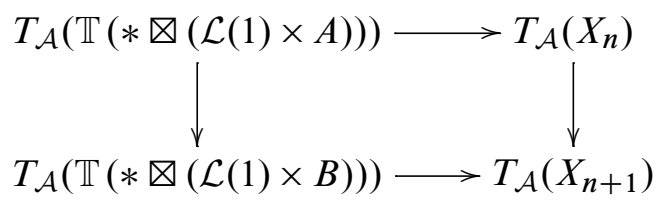

where $X_{n}$ can be assumed to be in the class $\overline{\mathcal{F}_{S}}$ and $A$ and $B$ are CW-complexes. Here we are suppressing the maps from $A, B, X_{n}$, and $X_{n+1}$ to $B G_{\mathcal{A}}$ in our notation. We need to show that under these hypotheses $T_{\mathcal{A}} X_{n+1}$ is in the class $\overline{\mathcal{F}_{S}}$. Next, since both $T_{\mathcal{A}}$ and $\mathbb{T}$ preserves Hurewicz cofibrations and $\overline{\mathcal{F}_{S}}$ is closed under pushouts along Hurewicz cofibrations, it suffices to show that for any CW-complex $Z$, $T_{\mathcal{A}}(\mathbb{T}(* \otimes(\mathcal{L}(1) \times Z)))$ is in the class $\overline{\mathcal{F}_{S}}$. Since $T(Z)$ has the homotopy type of a CW-spectrum (see the proof of $[23,7.5 .6]$ ), the result follows from Proposition 4.20 and Theorem 5.2.

This completes the verification of (A3). For (A4), we use the functor $Q$ of Definition 4.12; we have already verified that it has the desired properties in Proposition 4.26. For (A5), we choose a cofibrant replacement functor on the category of commutative monoids provided by the model structure of Theorem 4.18 and use this to define $B G_{\mathcal{A}}^{\prime}$. (Recall from the discussion prior to Proposition 4.24 that $B G_{\mathcal{A}}^{\prime}$ has the desired properties.)

Finally, rectification is very straightforward in this context; since Proposition 4.7 tells us that a map $X \rightarrow B G_{\mathcal{A}}$ over the non- $\Sigma$ linear isometries operad specifies the data of a monoid map in $\left(\mathcal{U}[\mathbb{L}] / B G_{\mathcal{A}}\right)[\mathbb{T}]$, by applying $* \otimes(-)$ we obtain a monoid map in $*$-modules. To complete the verification of (A6), we use Proposition 4.26. Given a topological monoid $M$, we can regard this as an $A_{\infty}$ space over the non- $\Sigma$ linear isometries operad by pulling back along the augmentation to the associative operad. Equivalently, $M$ regarded as an $\mathcal{L}(1)$-space with trivial action is a $\nabla_{\mathcal{L}}$-monoid with multiplication induced from the monoid multiplication $M \times M \rightarrow M$ and the fact that $M \otimes_{\mathcal{L}} M \cong M \times M$ by the argument of Lemma 4.13.

Thus, let $X$ be an $A_{\infty}$ space structured by the non- $\Sigma$ linear isometries operad. Since $Q$ is left adjoint to the functor which assigns the trivial $\mathcal{L}(1)$-action, the unit of the adjunction induces a map of $\mathcal{L}(1)$-spaces $X \otimes_{\mathcal{L}} X \rightarrow Q\left(X \otimes_{\mathcal{L}} X\right) \cong Q X \times Q X$. To show that Axiom (A6) holds, it suffices to show that this is a map of $\nabla_{\mathcal{L}}$-monoids; this map constructs the homotopy commutative diagram of the axiom. But since 
$Q X \times Q X \cong Q X \nabla_{\mathcal{L}} Q X$ this map is a map of $\nabla_{\mathcal{L}}$-monoids by the definition of the multiplication on $Q X$.

\section{Modifications when working over $B F$}

In this section, we discuss the situation when working over $B F$ : there are technical complications which arise from the fact that the projection

$$
p_{n}: B\left(*, F(n), S^{n}\right) \rightarrow B(*, F(n), *)
$$

is a universal quasifibration, with section a Hurewicz cofibration. Quasifibrations are not preserved under pullback, and in general the pullback of the section will not be a Hurewicz cofibration. If the section of $p_{n}$ could be shown to be a fiberwise cofibration, pullback along any map would provide a section which was a fiberwise cofibration [36, Section 5]. Unfortunately, this seems difficult: the proof that the section is a Hurewicz cofibration depends on the facts that the spaces in question are LEC and retractions between LEC spaces are cofibrations.

\subsection{A review of the properties of $\Gamma$}

The standard solution to these issues (pioneered by Lewis) is to use an explicit functor $\Gamma$ which replaces a map by a Hurewicz fibration. Since various properties of $\Gamma$ play an essential role in our work in this section, we will review relevant details here (see [23, Section 7.1] for a more comprehensive treatment).

Definition 6.1 Given a map $f: X \rightarrow B$, define

$$
\Pi B=\left\{(\theta, r) \in B^{[0, \infty]} \times[0, \infty] \mid \theta(t)=\theta(r), t>r\right\} .
$$

The end-point projection $v: \Pi B \rightarrow B$ is defined as $(\theta, r) \mapsto \theta(r)$. There is also the evaluation map $e_{0}: \Pi B \rightarrow B$ defined as $(\theta, r) \mapsto \theta(0)$. Define $\Gamma X$ to be the pullback

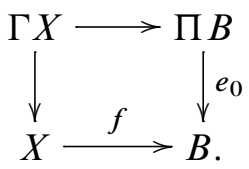

Let $\Gamma f$ denote the induced map

$$
\Gamma X \longrightarrow \Pi B \stackrel{v}{\longrightarrow} B .
$$

There is a map $\delta: X \rightarrow \Gamma X$ specified by taking $x \in X$ to the pair $\left(x, \zeta_{f(x)}\right)$ where $\zeta_{f(x)}$ is the path of length zero at $f(x)$.

The map $\Gamma f$ is a Hurewicz fibration. 
Lemma 6.2 The maps $\delta$ specify a natural transformation id $\rightarrow \Gamma$, and $\delta: X \rightarrow \Gamma X$ is a homotopy equivalence (although not a homotopy equivalence over $B$ ).

Moreover, $\Gamma$ has very useful properties in terms of interaction with the naive model structures on $\mathcal{U} / B$ and $\mathcal{T} / B[23,7.1 .11]$.

Proposition 6.3 The functor $\Gamma$ on spaces over $B$ takes cofibrations to fiberwise cofibrations and homotopy equivalences over $B$ to fiberwise homotopy equivalences. As a functor on ex-spaces, it takes ex-spaces with sections which are cofibrations to ex-spaces with sections which are fiberwise cofibrations.

There is a useful related lemma.

Lemma 6.4 If $X \rightarrow X^{\prime}$ is a weak equivalence over $B F, \Gamma X \rightarrow \Gamma X^{\prime}$ is a weak equivalence.

There are two possible ways we might use $\Gamma$ to resolve the problems with $B F$; we could replace $p_{n}$ with $\Gamma p_{n}$, which will be a Hurewicz fibration and will have section a fiberwise cofibration, or we could replace a given map $f: X \rightarrow B F$ with a Hurewicz fibration via $\Gamma$. The latter approach will yield a homotopically well-behaved Thom spectrum construction, since the pullback of a quasifibration along a Hurewicz fibration is a quasifibration and the pullback of a section which is a cofibration will be a cofibration. Moreover, Lewis shows that the two approaches yield stably equivalent Thom spectra. Since the second approach is much more felicitous for the study of multiplicative structures, we will employ it exclusively.

$\Gamma$ behaves well with respect to colimits and unbased tensors [23, 7.1.9].

Proposition 6.5 As a functor on $\mathcal{U} / B F$ and $\mathcal{T} / B F, \Gamma$ commutes with colimits.

There is a related result for tensors with unbased spaces (although note however that this is false for based tensors).

Proposition 6.6 As a functor on $\mathcal{U} / B F$ and $\mathcal{T} / B F, \Gamma$ commutes with the tensor with an unbased space $A$.

Finally, we recall some salient facts about the interaction of $\Gamma$ with operadic multiplications. May $[32,1.8]$ shows that $\Gamma$ restricts to a functor on $\mathcal{T}[\mathbb{C}]$, for an operad $\mathcal{C}$ augmented over the linear isometries operad, and Lewis observes that in fact $\Gamma$ extends to a functor on $\mathcal{T}[\mathbb{C}] / B F$. We make particular use of these facts in the cases of $\mathbb{T}$ and $\mathbb{P}$. A related observation we will use is that $\Gamma$ restricts to a functor on $\mathcal{U}[\mathbb{L}]$. An essential aspect of these results is that all of the various maps associated with $\Gamma$ (notably $\delta$ ) are maps of $\mathbb{C}$-algebras, and so in particular the map $\delta$ yields a weak equivalence in the category $\mathcal{T}[\mathbb{C}]$. 


\section{2 $\Gamma$ and cofibrant replacement}

In order to verify Axiom (A3) in this setting, we must amend the cofibrant replacement process. Given a map $f: X \rightarrow B F$ regarded as a map in $(\mathcal{U}[\mathbb{L}])[\mathbb{T}]$, we consider the map $\Gamma(c f): \Gamma(c X) \rightarrow B F$ obtained by the cofibrant replacement functor in $(\mathcal{U}[\mathbb{L}])[\mathbb{T}]$ followed by $\Gamma$. We have the following commutative diagram:

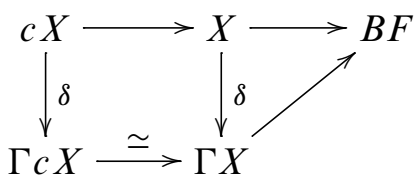

Since the labeled weak equivalence in the preceding diagram connect objects of $(\mathcal{U}[\mathbb{L}])[\mathbb{T}] / B F$ which are $T$-good, there is a stable equivalence connecting $T_{\mathcal{L}}(\Gamma f)$ to $\left.T_{\mathcal{L}}(\Gamma(c f))\right)$. If $f$ itself was $T$-good, then there is a further stable equivalence from $T_{\mathcal{L}} f$. Therefore this process does not change the homotopy type of the Thom spectrum. Finally, we apply $* \otimes(-)$ to ensure that we land in $\mathcal{M}_{*}[\mathbb{T}] / B G_{\mathcal{A}}$. Denote this composite functor by $\gamma$.

Next, we must verify that this process produces something which allows us to compute derived functors with respect to $\otimes$ and $\wedge$, in $\mathcal{M}_{*}[\mathbb{T}]$ and $\mathcal{M}_{S}[\mathbb{T}]$ respectively. Although $* \nabla \Gamma(c X)$ is not a cofibrant object in $\mathcal{M}_{*}[\mathbb{T}]$, it has the homotopy type of a cofibrant object and this suffices to ensure that it can be used to compute the derived $\triangle$ product. This observation also implies that the functor $Q$ satisfies Axiom (A4) in this context.

Moving on, we now need to show that $T_{\mathcal{A}}(* \otimes \Gamma(c f))$ can be used to compute the derived smash product in $\mathcal{M}_{S}$.

Lemma 6.7 Let $f: X \rightarrow B G_{\mathcal{A}}$ be a map in $\mathcal{M}_{*}[\mathbb{T}]$. Let $U$ denote the forgetful functors $\mathcal{M}_{*}[\mathbb{T}] \rightarrow \mathcal{M}_{*}$ and $\mathcal{M}_{S}[\mathbb{T}] \rightarrow \mathcal{M}_{S}$ respectively. Then there is an isomorphism $T_{\mathcal{A}}(U f) \cong U T_{\mathcal{A}}(f)$.

By Lemma 6.7, it will suffice to show that the underlying $S$-module of $T_{\mathcal{A}}(* \otimes \Gamma(c f))$ is in the class $\overline{\mathcal{F}_{S}}$. Since a slight modification of the argument of Proposition 4.24 shows that the underlying $\mathcal{L}(1)$-space of a cell $\nabla_{\mathcal{L}}$-monoid is a cell $\mathcal{L}(1)$-space, in fact Lemma 6.7 implies that it will suffice to show that given

$$
f: X \rightarrow B F_{\mathcal{A}}
$$

such that $X$ is a cell $\mathcal{L}(1)$-space, $T_{\mathcal{A}}(* \otimes \Gamma f)$ is in the class $\overline{\mathcal{F}_{S}}$. We will make an inductive argument. Using the fact that $\Gamma$ commutes with colimits in $\mathcal{L}(1)$-spaces and preserves Hurewicz cofibrations, it suffices to show that the Thom spectra of 
$* \otimes \Gamma \mathbb{T}\left(C E_{n}\right)$ and $* \otimes \Gamma \mathbb{T}\left(E_{n}\right)$ are in the class $\overline{\mathcal{F}_{S}}$, where $E_{n}$ is a wedge of cells $D^{n}$. We can further reduce to the case where we are considering a single cell. Abusing notation by suppressing the maps to $B F$, we will refer to the relevant Thom spectra as $T_{\mathcal{A}}\left(* \otimes \Gamma\left(\mathcal{L}(1) \times S^{n}\right)\right)$ and $T_{\mathcal{A}}\left(* \otimes \Gamma\left(\mathcal{L}(1) \times D^{n}\right)\right)$. Finally, since $S^{n}$ can be constructed as the pushout $D^{n} \cup_{S^{n-1}} D^{n}$, it suffices to consider $T_{\mathcal{A}}(* \otimes \Gamma(\mathcal{L}(1) \times *))$ and $T_{\mathcal{A}}\left(* \otimes \Gamma\left(\mathcal{L}(1) \times D^{n}\right)\right)$. Recall that by Proposition 5.6, these spectra are isomorphic as $S$-modules to $S \wedge_{\mathcal{L}} T_{\mathcal{L}}(\Gamma(\mathcal{L}(1) \times *))$ and $S \wedge_{\mathcal{L}} T_{\mathcal{L}}\left(\Gamma\left(\mathcal{L}(1) \times D^{n}\right)\right)$ respectively.

Lemma 6.8 The $S$-modules $T_{\mathcal{A}}(* \otimes \Gamma(\mathcal{L}(1) \times *))$ and $T_{\mathcal{A}}\left(* \otimes \Gamma\left(\mathcal{L}(1) \times D^{n}\right)\right)$ are in the class $\overline{\mathcal{F}_{S}}$.

Proof Given a map $D^{n} \rightarrow B F$, by choosing a point in $D^{n}$ we obtain a map $* \rightarrow D^{n}$ over $B F$. This induces a map $\mathcal{L}(1) \times * \rightarrow \mathcal{L}(1) \times D^{n}$ which is a weak equivalence of $\mathcal{L}(1)$-spaces over $B F$. Since these are cofibrant $\mathcal{L}(1)$-spaces, this is a homotopy equivalence over $B F$. Applying $\Gamma$ turns this into a fiberwise homotopy equivalence. Since $T$ takes fiberwise homotopy equivalences to homotopy equivalences of spectra, the resulting spectra are homotopy equivalent.

Therefore, we are reduced to considering the Thom spectra associated to $\Gamma(\mathcal{L}(1) \times *)$ associated to the various choices of a target for point. But since $B F$ is path-connected, an argument analogous to the one in the preceding paragraph allows us to show that all of these spectra are homotopy equivalent. Thus, it suffices to consider the trivial map $* \rightarrow B F$. But then $\Gamma(\mathcal{L}(1) \times *))$ is homeomorphic as a space over $B F$ to $\pi_{2}: \mathcal{L}(1) \times \Gamma(*)$, where $\pi_{2}$ is the projection away from $\mathcal{L}(1)$. Applying $T$ yields the Thom spectrum $\mathcal{L}(1)_{+} \wedge T(\Gamma(*))$. Finally, $S \wedge_{\mathcal{L}}\left(\mathcal{L}(1)_{+} \wedge T(\Gamma(*))\right.$ is in the class $\overline{\mathcal{F}_{S}}$; this follows from the proof of part (ii) of [23, 7.3.7], in which the homotopy type of the Thom spectrum $T(\Gamma(*))$ is explicitly described.

In the previous proof, we are implicitly exploiting the "untwisting" Proposition I.2.1 of [16] which provides an isomorphism of spectra $A \ltimes \Sigma^{\infty} X \cong \Sigma^{\infty}\left(A_{+} \wedge X\right)$.

Finally, we need to be able to compare $\Gamma\left(f \otimes_{\mathcal{L}} f\right)$ to $\Gamma f \otimes_{\mathcal{L}} \Gamma f$.

Proposition 6.9 Let $f: X \rightarrow B F$ be a map of $\mathcal{L}(1)$-spaces, and assume that $X$ is a cell $\mathcal{L}(1)$-space. Then there is a weak equivalence between $T_{\mathcal{L}} \Gamma\left(f \otimes_{\mathcal{L}} f\right)$ and $T_{\mathcal{L}}\left(\Gamma f \otimes_{\mathcal{L}} \Gamma f\right)$.

Proof Recall from Proposition 4.20 that given a choice of a linear isometric isomorphism $g: \mathbb{U}^{2} \rightarrow \mathbb{U}$, there is a chain of weak equivalences

$$
X \times X \rightarrow \mathcal{L}(2) \times(X \times X) \rightarrow X \otimes_{\mathcal{L}} X .
$$

Moreover, these equivalences are given by maps over $B F$. 
Lewis shows that there is a map $\Gamma f \times \Gamma f \rightarrow \Gamma(f \times f)$ given by multiplication of paths which is a weak equivalence [23, 7.5.5]. In addition, he shows that $\Gamma f \times \Gamma f$ is a good map. This is the heart of our comparison. Applying $\Gamma$ to the chain of equivalences above, we have a composite

$$
\Gamma(f \times f) \rightarrow \Gamma(\mathcal{L}(2) \times(f \times f)) \rightarrow \Gamma\left(f \otimes_{\mathcal{L}} f\right)
$$

which induces weak equivalences of Thom spectra $T(\Gamma(f \times f)) \rightarrow T\left(\Gamma\left(f \otimes_{\mathcal{L}} f\right)\right)$. On the other hand, there is also the composite

$$
\Gamma f \times \Gamma f \rightarrow \mathcal{L}(2) \times(\Gamma f \times \Gamma f) \rightarrow \Gamma f \otimes_{\mathcal{L}} \Gamma f .
$$

Although these are not all good maps, Lemma 6.8 implies that the induced maps of Thom spectra

$$
g_{*}(T(\Gamma f) \bar{\wedge} T(\Gamma f)) \rightarrow \mathcal{L}(2) \ltimes(T(\Gamma f) \bar{\wedge} T(\Gamma f)) \rightarrow T(\Gamma f) \otimes_{\mathcal{L}} T(\Gamma f)
$$

are stable equivalences. Since $\Gamma f \times \Gamma f \rightarrow \Gamma(f \times f)$ induces a weak equivalence of Thom spectra, the result follows.

The previous proposition gives us the following result, which allows us to compare to a model of the free loop space which is $T$-good in the proof of the main theorem from the axioms.

Corollary 6.10 Let $f: X \rightarrow B G_{\mathcal{A}}$ be a map of $\otimes$-monoids. Then there is a weak equivalence of spectra $T_{\mathcal{A}}\left(N_{\bigotimes}^{\mathrm{cy}}(\gamma f)\right)$ and $T_{\mathcal{A}}\left(\gamma N_{\bigotimes}^{\mathrm{cy}} f\right)$.

Finally, the verification of Axioms (A5) and (A6) is unchanged.

\section{Preliminaries on symmetric spectra}

Let $\mathrm{Sp}^{\Sigma}$ be the category of topological symmetric spectra as defined in [31]. Thus, a symmetric spectrum $T$ is a spectrum in which the spaces $T(n)$ come equipped with base point preserving $\Sigma_{n}$-actions such that the iterated structure maps $S^{m} \wedge T(n) \rightarrow$ $T(m+n)$ are $\left(\Sigma_{m} \times \Sigma_{n}\right)$-equivariant. It is proved in [31] that $\mathrm{Sp}^{\Sigma}$ has a stable model structure which makes it Quillen equivalent to the category Sp of spectra. When implementing the axiomatic framework in this setting there are two technical issues that must be addressed. The first is that the forgetful functor from $\mathrm{Sp}^{\Sigma}$ to $\mathrm{Sp}$ does not take stable model equivalences in $\mathrm{Sp}^{\Sigma}$ (that is, the weak equivalences in the stable model structure) to ordinary stable equivalences in Sp. This can be remedied using Shipley's detection functor as we recall below. The second issue is that the symmetric 
Thom spectrum functor on $\mathcal{I U} / B F$ does not take cofibrant replacement in the model structure on $\mathcal{I U}$ to cofibrant replacement in $\mathrm{Sp}^{\Sigma}$. For this reason we shall introduce explicit "flat replacement" functors on $\mathcal{I U}$ and $\mathrm{Sp}^{\Sigma}$ which are strictly compatible with the symmetric Thom spectrum functor. In order for this to be useful, we must of course verify that the topological Hochschild homology of a symmetric ring spectrum is represented by the cyclic bar construction of its flat replacement; this is the content of Proposition 7.6 and Proposition 7.10.

\subsection{The detection functor}

A map of symmetric spectra whose underlying map of spectra is an ordinary stable equivalence is also a stable model equivalence in $\mathrm{Sp}^{\Sigma}$. It is a subtle property of the stable model structure on $\mathrm{Sp}^{\Sigma}$ that the converse does not hold; there are stable model equivalences in $\mathrm{Sp}^{\Sigma}$ whose underlying maps of spectra are not stable equivalences. In order to characterize the stable model equivalences in terms of ordinary stable equivalences, Shipley [46] has defined an explicit "detection" functor $D: \mathrm{Sp}^{\Sigma} \rightarrow \mathrm{Sp}^{\Sigma}$. This functor takes a symmetric spectrum $T$ to the symmetric spectrum $D T$ with $n$-th space

$$
D T(n)=\underset{\mathbf{m} \in \mathcal{I}}{\operatorname{hocolim}} \Omega^{m}\left(T(m) \wedge S^{n}\right) .
$$

Here we tacitly replace the spaces in the definition of $D T$ by spaces that are well-based, for example the realization of their singular simplicial complexes. It then follows as in $[46,3.1 .2]$, that a map of symmetric spectra is a stable model equivalence if and only if applying $D$ gives an ordinary stable equivalence of the underlying spectra. Furthermore, by [46, 3.1.6], the functor $D$ is related to the identity functor on $\mathrm{Sp}^{\Sigma}$ by a chain of natural stable model equivalences of symmetric spectra.

\subsection{The flatness condition for symmetric spectra}

It follows from [46], that if $T$ is a cofibrant symmetric ring spectrum, then the cyclic bar construction $B^{\text {cy }}(T)$ represents the topological Hochschild homology of $T$. However, in the study of Thom spectra we find it useful to introduce the notion of a flat symmetric spectrum, which is a more general type of symmetric spectrum for which the smash product is homotopically well-behaved. We first consider flat symmetric spectra in general and then define what we mean by a flat symmetric ring spectrum. For this we need to recall some convenient notation from [42]. In the following $T$ denotes a symmetric spectrum and $\mathcal{I}$ is the category of finite sets and injective maps defined in the introduction. Given a morphism $\alpha: \mathbf{m} \rightarrow \mathbf{n}$, we write $\mathbf{n}-\alpha$ for the set $\mathbf{n}-\alpha(\mathbf{m})$ and $S^{n-\alpha}$ for the one-point compactification of $\mathbb{R}^{\mathbf{n}-\alpha}$. Associated to $\alpha$ we have the 
composite map

$$
S^{n-\alpha} \wedge T(m) \longrightarrow S^{n-m} \wedge T(m) \longrightarrow T(n) \stackrel{\bar{\alpha}}{\rightarrow} T(n),
$$

where the first map is the homeomorphism induced by the ordering of $\mathbf{n}-\alpha$ inherited from $\mathbf{n}$, the second map is the structure map of the symmetric spectrum, and $\bar{\alpha}$ is the extension of $\alpha$ to a permutation which is order preserving on the complement of $\mathbf{m}$. The advantage of this notation is that it will make some of our constructions self-explanatory. Consider for each object $\mathbf{n}$ the $\mathcal{I} / \mathbf{n}$-diagram of based spaces that to an object $\alpha: \mathbf{m} \rightarrow \mathbf{n}$ associates $S^{n-\alpha} \wedge T(m)$. If $\beta:(\mathbf{m}, \alpha) \rightarrow\left(\mathbf{m}^{\prime}, \alpha^{\prime}\right)$ is a morphism in $\mathcal{I} / \mathbf{n}$, then $\alpha=\alpha^{\prime} \circ \beta$ by definition, and $\beta$ specifies a canonical homeomorphism between $S^{n-\alpha}$ and $S^{n-\alpha^{\prime}} \wedge S^{m^{\prime}-\beta}$. The induced map is then defined by

$$
S^{n-\alpha} \wedge T(m) \stackrel{\simeq}{\rightarrow} S^{n-\alpha^{\prime}} \wedge S^{m^{\prime}-\beta} \wedge T(m) \longrightarrow S^{n-\alpha^{\prime}} \wedge T\left(m^{\prime}\right) .
$$

Applying this functor to a commutative diagram in $\mathcal{I}$ of the form

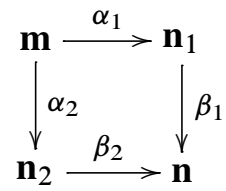

and writing $\gamma$ for the composition $\beta_{1} \circ \alpha_{1}=\beta_{2} \circ \alpha_{2}$, we get a commutative diagram of based spaces

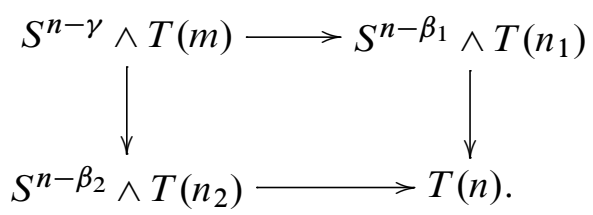

We say that $T$ is flat if each of the spaces $T(n)$ is well-based, and if for each diagram (7-1), such that the intersection of the images of $\beta_{1}$ and $\beta_{2}$ equals the image of $\gamma$, the induced map

$$
S^{n-\beta_{1}} \wedge T\left(n_{1}\right) \cup_{S^{n-\gamma} \wedge T(m)} S^{n-\beta_{2}} \wedge T\left(n_{2}\right) \longrightarrow T(n)
$$

is an $h$-cofibration (that is, it has the homotopy extension property in the usual sense). By Lillig's union theorem for $h$-cofibrations [24], a levelwise well-based symmetric spectrum $T$ is flat if and only if (i) any morphism $\alpha: \mathbf{m} \rightarrow \mathbf{n}$ in $\mathcal{I}$ induces an $h-$ cofibration $S^{n-\alpha} \wedge T(m) \rightarrow T(n)$, and (ii) for any diagram of the form (7-1), satisfying the above condition, the intersection of the images of $S^{n-\beta_{1}} \wedge T\left(n_{1}\right)$ and $S^{n-\beta_{2}} \wedge T\left(n_{2}\right)$ equals the image of $S^{n-\gamma} \wedge T(m)$. This can be reformulated in a way that is easier to check in practice. 
Lemma 7.1 A levelwise well-based symmetric spectrum is flat if and only if the structure maps $S^{1} \wedge T(n) \rightarrow T(n+1)$ are $h$-cofibrations and the diagrams

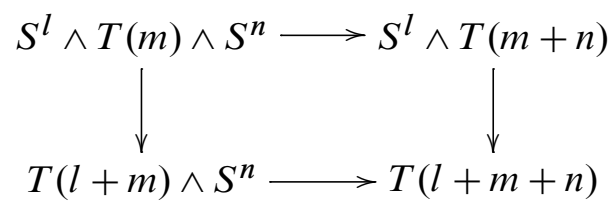

are pullback diagrams for all $l, m$ and $n$.

Here the notation is supposed to be self-explanatory. For instance, the symmetric Thom spectra $M O$ and $M F$ obtained by applying the Thom space functor levelwise are flat. We shall now prove that smash products of flat symmetric spectra are homotopically well-behaved. Recall from [42] that the smash product of a family of symmetric spectra $T_{1}, \ldots, T_{k}$ may be identified with the symmetric spectrum whose $n$-th space is the colimit

$$
T_{1} \wedge \cdots \wedge T_{k}(n)=\operatorname{colim}_{\alpha: \mathbf{n}_{1} \sqcup \cdots \sqcup \mathbf{n}_{k} \rightarrow \mathbf{n}} S^{n-\alpha} \wedge T_{1}\left(n_{1}\right) \wedge \cdots \wedge T_{k}\left(n_{k}\right) .
$$

Here the colimit is over the comma category $\sqcup^{k} / \mathbf{n}$, where $\sqcup^{k}: \mathcal{I}^{k} \rightarrow \mathcal{I}$ denotes the iterated monoidal product. We introduce a homotopy invariant version by the analogous based homotopy colimit construction,

$$
T_{1} \wedge^{h} \cdots \wedge^{h} T_{k}(n)=\underset{\alpha: \mathbf{n}_{1} \sqcup \cdots \sqcup \mathbf{n}_{k} \rightarrow \mathbf{n}}{\operatorname{hocolim}} S^{n-\alpha} \wedge T_{1}\left(n_{1}\right) \wedge \cdots \wedge T_{k}\left(n_{k}\right) .
$$

For $k=1$ this is the replacement $\bar{T}_{1}$ to be considered in Section 7.3. It should be observed that the functor on $k$-tuples of symmetric spectra so defined is not the same as the iteration of the functor $(-) \wedge^{h}(-)$. As we shall see in Proposition 7.3, the symmetric spectrum $T_{1} \wedge^{h} \cdots \wedge^{h} T_{k}$ always represents the "derived" homotopy type of the smash product for symmetric spectra that are levelwise well-based.

Proposition 7.2 If the symmetric spectra $T_{1}, \ldots, T_{k}$ are flat, then the canonical projection

$$
T_{1} \wedge^{h} \cdots \wedge^{h} T_{k} \longrightarrow T_{1} \wedge \cdots \wedge T_{k}
$$

is a levelwise equivalence.

Proof For notational reasons we only carry out the proof for a pair of flat symmetric spectra $T_{1}$ and $T_{2}$. The proof in the general case is completely analogous. Let $\mathcal{A}(n)$ be the full subcategory of $\sqcup / \mathbf{n}$ whose objects $\alpha: \mathbf{n}_{1} \sqcup \mathbf{n}_{2} \rightarrow \mathbf{n}$ are such that the restrictions 
to $\mathbf{n}_{1}$ and $\mathbf{n}_{2}$ are order preserving. Since this is a skeleton subcategory, it suffices to show that the canonical map

$$
\underset{\mathcal{A}(n)}{\operatorname{hocolim}} S^{n-\alpha} \wedge T_{1}\left(n_{1}\right) \wedge T_{2}\left(n_{2}\right) \longrightarrow \operatorname{colim}_{\mathcal{A}(n)} S^{n-\alpha} \wedge T_{1}\left(n_{1}\right) \wedge T_{2}\left(n_{2}\right)
$$

is a weak homotopy equivalence for each $n$. Notice that $\mathcal{A}(n)$ may be identified with the partially ordered set of pairs $\left(U_{1}, U_{2}\right)$ of disjoint subsets of $\mathbf{n}$, so that we may write the diagram in the form

$$
Z\left(U_{1}, U_{2}\right)=S^{\mathbf{n}-U_{1} \cup U_{2}} \wedge T_{1}\left(U_{1}\right) \wedge T_{2}\left(U_{2}\right) .
$$

Notice also, that since the base points are nondegenerate and the categories $\mathcal{A}(n)$ are contractible, it suffices to consider the unbased homotopy colimit instead of the based homotopy colimit. We now use that the categories $\mathcal{A}(n)$ are very small in the sense of [15, Section 10.13]. By general model theoretical arguments using the Strøm model category structure [48] on $\mathcal{U}$, we are therefore left with showing that the canonical map

$$
\underset{\left(U_{1}, U_{2}\right) \subsetneq\left(U_{1}^{0}, U_{2}^{0}\right)}{\operatorname{colim}} Z\left(U_{1}, U_{2}\right) \longrightarrow Z\left(U_{1}^{0}, U_{2}^{0}\right)
$$

is an $h$-cofibration for each fixed object $\left(U_{1}^{0}, U_{2}^{0}\right)$. Since the structure maps in the $\mathcal{A}(n)$-diagram $Z$ are $h$-cofibrations and since $h$-cofibrations are closed inclusions, we may view each of the spaces $Z\left(U_{1}, U_{2}\right)$ as a subspace of $Z\left(U_{1}^{0}, U_{2}^{0}\right)$. By the flatness assumptions on $T_{1}$ and $T_{2}$ we then have the equality

$$
Z\left(U_{1}, U_{2}\right) \cap Z\left(V_{1}, V_{2}\right)=Z\left(U_{1} \cap V_{1}, U_{2} \cap V_{2}\right)
$$

for each pair of objects $\left(U_{1}, U_{2}\right)$ and $\left(V_{1}, V_{2}\right)$. Thus, by the pasting lemma [37, 18.3] for maps defined on a union of closed subspaces, the colimit in question may be identified with the union of the subspaces $Z\left(U_{1}, U_{2}\right)$. The conclusion now follows from an inductive argument using Lillig's union theorem for $h$-cofibrations [24].

Proposition 7.3 If $T_{1}, \ldots, T_{k}$ are levelwise well-based symmetric spectra, then there is a chain of levelwise equivalences

$$
T_{1} \wedge^{h} \cdots \wedge^{h} T_{k} \stackrel{\simeq}{\longleftarrow} T_{1}^{\prime} \wedge^{h} \cdots \wedge^{h} T_{k}^{\prime} \stackrel{\simeq}{\rightarrow} T_{1}^{\prime} \wedge \cdots \wedge T_{k}^{\prime},
$$

where $T_{i}^{\prime} \rightarrow T_{i}$ are cofibrant replacements in the stable model structure on symmetric spectra.

Proof It follows from $[31,9.9]$ that we may choose cofibrant symmetric spectra $T_{i}^{\prime}$ and levelwise acyclic fibrations $T_{i}^{\prime} \stackrel{\simeq}{\rightarrow} T_{i}$. The left hand map is then a levelwise equivalence since homotopy colimits preserve termwise equivalences of well-based diagrams. That the right hand map is an equivalence follows from Proposition 7.2 since 
cofibrant symmetric spectra are retracts of relative cell-complexes by [31], hence in particular flat.

Combining these propositions we get the following corollary which states that smash products of flat symmetric spectra represent the "derived" smash products.

Corollary 7.4 If $T_{1}, \ldots, T_{k}$ are flat symmetric spectra, then there is a levelwise equivalence

$$
T_{1}^{\prime} \wedge \cdots \wedge T_{k}^{\prime} \stackrel{\simeq}{\rightarrow} T_{1} \wedge \cdots \wedge T_{k},
$$

where $T_{i}^{\prime} \rightarrow T_{i}$ are cofibrant replacements in the stable model structure on symmetric spectra.

In the next definition we use the notion of an $h$-cofibration introduced in Section 3.1.

Definition 7.5 A flat symmetric ring spectrum $T$ is a symmetric ring spectrum whose underlying symmetric spectrum is flat and whose unit $S \rightarrow T$ is an $h$-cofibration.

Proposition 7.6 If $T$ is a flat symmetric ring spectrum, then $B^{\text {cy }}(T)$ represents the topological Hochschild homology of $T$.

Proof Let $T^{\prime} \rightarrow T$ be a cofibrant replacement of $T$ as a symmetric ring spectrum. Then it follows from [46] that $B^{\text {cy }}\left(T^{\prime}\right)$ represents the topological Hochschild homology of $T$. Using Corollary 7.4, we see that the induced map of simplicial symmetric spectra $B_{\bullet}^{\mathrm{cy}}\left(T^{\prime}\right) \rightarrow B_{\bullet}^{\mathrm{cy}}(T)$ is a levelwise equivalence in each simplicial degree. Furthermore, the assumption that the unit be an $h$-cofibration implies by Lemma 3.2 that these are good simplicial spaces. Therefore the topological realizations are also levelwise equivalent.

\subsection{Flat replacement of symmetric spectra}

We define an endofunctor on the category of symmetric spectra by associating to a symmetric spectrum $T$ the symmetric spectrum $\bar{T}$ defined by

$$
\bar{T}(n)=\underset{\alpha: \mathbf{n}_{1} \rightarrow \mathbf{n}}{\operatorname{hocolim}} S^{n-\alpha} \wedge T\left(n_{1}\right),
$$

where the (based) homotopy colimit is over the category $\mathcal{I} / \mathbf{n}$. This is not quite a flat replacement functor since $\bar{T}$ need not be levelwise well-based. However, we do have the following.

Proposition 7.7 If $T$ is a symmetric spectrum that is levelwise well-based, then $\bar{T}$ is flat and the canonical projection $\bar{T} \rightarrow T$ is a levelwise weak equivalence. 
Proof We show that $\bar{T}$ satisfies the flatness conditions (i) and (ii). Thus, given a morphism $\alpha: \mathbf{m} \rightarrow \mathbf{n}$, we claim that the structure map $S^{n-\alpha} \wedge \bar{T}(m) \rightarrow \bar{T}(n)$ is an $h$-cofibration. Let $\alpha_{*}: \mathcal{I} / \mathbf{m} \rightarrow \mathcal{I} / \mathbf{n}$ be the functor induced by $\alpha$. Using that based homotopy colimits commute with smash products and that there is a natural isomorphism of $\mathcal{I} / \mathbf{m}$-diagrams

$$
S^{n-\alpha} \wedge S^{m-\beta} \wedge T\left(m_{1}\right) \cong S^{n-\alpha \beta} \wedge T\left(m_{1}\right), \quad \beta: \mathbf{m}_{1} \longrightarrow \mathbf{m},
$$

the map in question may be identified with the map of homotopy colimits

$$
\underset{\beta: \mathbf{m}_{1} \rightarrow \mathbf{m}}{\operatorname{hocolim}} S^{n-\alpha \beta} \wedge T\left(m_{1}\right) \stackrel{\alpha_{*}}{\rightarrow} \underset{\gamma: \mathbf{n}_{1} \rightarrow \mathbf{n}}{\operatorname{hocolim}} S^{n-\gamma} \wedge T\left(n_{1}\right) .
$$

Notice that $\alpha_{*}$ induces an isomorphism of $\mathcal{I} / \mathbf{m}$ onto a full subcategory of $\mathcal{I} / \mathbf{n}$. The claim therefore follows from the general fact that the map of homotopy colimits obtained by restricting a diagram to a full subcategory is an $h$-cofibration; see eg Elmendorf et al [16, X.3.5]. In order to verify (ii) one first checks the condition in each simplicial degree of the simplicial spaces defining the homotopy colimits. The result then follows from the fact that topological realization preserves pullback diagrams. The map $\bar{T} \rightarrow T$ is defined by the canonical projection from the homotopy colimit to the colimit

$$
\bar{T}(n)=\underset{\mathcal{I} / \mathbf{n}}{\operatorname{hocolim}} T \longrightarrow \underset{\mathcal{I} / \mathbf{n}}{\operatorname{colim}} T=T(n),
$$

where the identification of the colimit comes from the fact that $\mathcal{I} / \mathbf{n}$ has a terminal object. The existence of a terminal object implies that this is a weak homotopy equivalence. $\square$

The relationship between the replacement functor and the $\wedge^{h}$-product is recorded in the following proposition whose proof we leave with the reader.

Proposition 7.8 There is a natural isomorphism of symmetric spectra

$$
\bar{T}_{1} \wedge \cdots \wedge \bar{T}_{k} \cong T_{1} \wedge^{h} \cdots \wedge^{h} T_{k} .
$$

Recall the notion of a monoidal functor from [25, Section XI.2]. This is what is sometimes called lax monoidal.

Proposition 7.9 The replacement functor $T \mapsto \bar{T}$ is (lax) monoidal and the canonical map $\bar{T} \rightarrow T$ is a monoidal natural transformation.

Proof The replacement of the sphere spectrum has 0th space $\bar{S}(0)=S^{0}$ and we let $S \rightarrow \bar{S}$ be the unique map of symmetric spectra that is the identity in degree 0 . We must define an associative and unital natural transformation $\bar{T}_{1} \wedge \bar{T}_{2} \rightarrow \overline{T_{1} \wedge T_{2}}$, which 
by the universal property of the smash product amounts to an associative and unital natural transformation of $\mathcal{I}_{S} \times \mathcal{I}_{S}$-diagrams

$$
\bar{T}_{1}(m) \wedge \bar{T}_{2}(n) \longrightarrow \overline{T_{1} \wedge T_{2}}(m+n) .
$$

Here $\mathcal{I}_{S}$ denotes the topological category such that $\mathrm{Sp}^{\Sigma}$ may be identified with the category of based $\mathcal{I}_{S}$-diagrams $[31 ; 42]$. Consider the natural transformation of $\mathcal{I} / \mathbf{m} \times \mathcal{I} / \mathbf{n}$-diagrams that to a pair of objects $\alpha: \mathbf{m}_{1} \rightarrow \mathbf{m}$ and $\beta: \mathbf{n}_{1} \rightarrow \mathbf{n}$ associates the map

$$
S^{m-\alpha} \wedge T_{1}\left(m_{1}\right) \wedge S^{n-\beta} \wedge T_{2}\left(n_{1}\right) \longrightarrow S^{m+n-\alpha \sqcup \beta} \wedge\left(T_{1} \wedge T_{2}\right)\left(m_{1}+n_{1}\right),
$$

where we first permute the factors and then apply the universal map to the smash product $T_{1} \wedge T_{2}$. Using that based homotopy colimits commute with smash products, the map (7-3) is the induced map of homotopy colimits, followed by the map

$$
\underset{\mathcal{I} / \mathbf{m} \times \mathcal{I} / \mathbf{n}}{\operatorname{hocolim}} S^{m+n-\alpha \sqcup \beta} \wedge\left(T_{1} \wedge T_{2}\right)\left(m_{1}+n_{1}\right) \longrightarrow \overline{T_{1} \wedge T_{2}}(m+n)
$$

induced by the concatenation functor $\mathcal{I} / \mathbf{m} \times \mathcal{I} / \mathbf{n} \rightarrow \mathcal{I} / \mathbf{m} \sqcup \mathbf{n}$. With this definition it is clear that the natural transformation $\bar{T} \rightarrow T$ is monoidal.

It follows from this that the replacement functor induces a functor on the category of symmetric ring spectra. In order to ensure that the unit is an $h$-cofibration we adapt the usual method for replacing a topological monoid with one that is well-based. Thus, given a symmetric ring spectrum $T$, we define $T^{\prime}$ to be the mapping cylinder

$$
T^{\prime}=T \cup_{S \wedge\{0\}_{+}} S \wedge I_{+},
$$

where we view the unit interval $I$ as a multiplicative topological monoid. This is again a symmetric ring spectrum and arguing as in the case of a topological monoid [32, A.8] one deduces that the unit $S \rightarrow T^{\prime}$ is an $h$-cofibration and that the canonical map of symmetric ring spectra $T^{\prime} \rightarrow T$ is a homotopy equivalence. It is easy to check that if $T$ is flat as a symmetric spectrum, then $T^{\prime}$ is a flat symmetric ring spectrum in the sense of Definition 7.5. Combining these remarks with Proposition 7.7, we get the following.

Proposition 7.10 If $T$ is a symmetric ring spectrum that is levelwise well-based, then $T^{c}=(\bar{T})^{\prime}$ is a flat symmetric ring spectrum. 


\section{Implementing the axioms for symmetric spectra}

In this section we verify the Axioms (A1)-(A6) in the setting of $\mathcal{I}$-spaces and symmetric spectra. The basic reference for this material is the third author's paper [42] in which the theory of Thom spectra is developed in the category of symmetric spectra.

\subsection{Symmetric spectra and $\mathcal{I}$-spaces}

In the axiomatic framework set up in Section 2.2 we define $\mathcal{S}$ to be the category of symmetric spectra $\operatorname{Sp}^{\Sigma}$ and we say that a symmetric ring spectrum is flat if it satisfies the conditions in Definition 7.5. We define $U$ to be the composite functor

$$
U: \mathrm{Sp}^{\Sigma} \stackrel{D}{\rightarrow} \mathrm{Sp}^{\Sigma} \longrightarrow \mathrm{Sp},
$$

where $D$ is the detection functor from Section 7.1 and the second arrow represents the obvious forgetful functor. It follows from the discussion in Section 7.1 that a map of symmetric spectra is a stable model equivalence if and only if applying $U$ gives an ordinary stable equivalence of spectra.

We define $\mathcal{A}$ to be the symmetric monoidal category $\mathcal{I U}$ of $\mathcal{I}$-spaces as defined in the introduction. Given an $\mathcal{I}$-space $B$, let $B[n]$ be the $\mathcal{I}$-space $B(\mathbf{n} \sqcup-)$ obtained by composing with the "shift" functor $\mathbf{n} \sqcup-$ on $\mathcal{I}$. We write $\operatorname{Hom}_{\mathcal{I}}(A, B)$ for the internal Hom-object in $\mathcal{I U}$ defined by $\mathbf{n} \mapsto \mathcal{I U}(A, B[n])$. This makes $\mathcal{I U}$ a closed symmetric monoidal topological category in the sense that there is a natural isomorphism

$$
\mathcal{I U}(A \otimes B, C) \cong \mathcal{I U}\left(A, \operatorname{Hom}_{\mathcal{I}}(B, C)\right) .
$$

We define an $\mathcal{I}$-space monoid to be a monoid in $\mathcal{I U}$. The tensor of an $\mathcal{I}$-space $A$ with a space $K$ is given by the obvious levelwise cartesian product $A \times K$. Associating to an $\mathcal{I}$-space its homotopy colimit over $\mathcal{I}$ defines the functor $U: \mathcal{I U} \rightarrow \mathcal{U}$, that is, $U A=\operatorname{hocolim}_{\mathcal{I}} A$. Here we adapt the Bousfield-Kan construction [10], such that by definition $U A$ is the realization of the simplicial space

$$
[k] \mapsto \coprod_{\mathbf{n}_{0} \leftarrow \cdots \leftarrow \mathbf{n}_{k}} A\left(n_{k}\right),
$$

where the coproduct is indexed over the nerve of $\mathcal{I}$. In particular, $U *$ is the classifying space $B \mathcal{I}$ which is contractible since $\mathcal{I}$ has an initial object. That $U$ preserves tensors and colimits follows from the fact that topological realization has this property. As in [42; 40] we also use the notation $A_{h \mathcal{I}}$ for the homotopy colimit of an $\mathcal{I}$-space $A$.

In the discussion of the Axioms (A1)-(A6) we consider two cases corresponding to the underlying Thom spectrum functor on $\mathcal{U} / B F$ and its restriction to $\mathcal{U} / B O$. In the case 
of (A2) and (A3) we formulate a slightly weaker version of the axioms which hold in the $\mathcal{I U} / B F$ case and which imply the original axioms when restricted to objects in $\mathcal{I U} / B O$. In Section 8.2 we then provide additional arguments to show why the weaker form of the axioms suffices to prove the statement in Theorem 1. One can also verify the axioms for more general families of subgroups of the topological monoids $F(n)$. We omit the details of this since the only reason for singling out the group valued case is to explain how the arguments simplify in this situation.

\section{Axiom (A1)}

As explained in Section 2.1, the correspondence $\mathbf{n} \mapsto B F(n)$ defines a commutative monoid in $\mathcal{I U}$. In order to be consistent with the notation used in [42], we now redefine $B F$ to be this $\mathcal{I}$-space monoid and we write $B F_{h \mathcal{I}}$ for its homotopy colimit. Let $\mathcal{N}$ be the subcategory of $\mathcal{I}$ whose only morphisms are the subset inclusions and let $i: \mathcal{N} \rightarrow \mathcal{I}$ be the inclusion. Thus, $\mathcal{N}$ may be identified with the ordered set of natural numbers. Let $B F_{h \mathcal{N}}$ be the homotopy colimit and $B F_{\mathcal{N}}$ the colimit of the $\mathcal{N}$-diagram $B F$, such that $B F_{\mathcal{N}}$ is now what was denoted $B F$ in Section 2.1. We then have a diagram of weak homotopy equivalences

$$
B F_{h \mathcal{I}} \stackrel{i}{\leftarrow} B F_{h \mathcal{N}} \stackrel{t}{\rightarrow} B F_{\mathcal{N}}
$$

where $t$ is the canonical projection from the homotopy colimit to the colimit. Here $i$ is a weak homotopy equivalence by Bökstedt's approximation lemma [26, 2.3.7], and $t$ is a weak homotopy equivalence since the structure maps are $h$-cofibrations. Using that $B F_{h \mathcal{I}}$ has the homotopy type of a $\mathrm{CW}$-complex, we choose a homotopy inverse $j$ of $i$ and define $\zeta$ to be the composite weak homotopy equivalence

$$
\zeta: B F_{h \mathcal{I}} \stackrel{j}{\rightarrow} B F_{h \mathcal{N}} \stackrel{t}{\rightarrow} B F_{\mathcal{N}} .
$$

Starting with the commutative $\mathcal{I}$-space monoid $\mathbf{n} \mapsto B O(n)$, we similarly get a weak homotopy equivalence $\zeta: B O_{h \mathcal{I}} \stackrel{\simeq}{\rightarrow} B O_{\mathcal{N}}$.

\section{Axiom (A2)}

The symmetric Thom spectrum functor

$$
T: \mathcal{I U} / B F \longrightarrow \mathrm{Sp}^{\Sigma}
$$

is defined by applying the Thom space functor from Section 2.1 levelwise: given an object $\alpha: A \rightarrow B F$ with level maps $\alpha_{n}: A(n) \rightarrow B F(n)$, the $n$-th space of the symmetric spectrum $T(\alpha)$ is given by $T\left(\alpha_{n}\right)$. Since colimits and tensors in $\mathcal{I U} / B F$ and $\mathrm{Sp}^{\Sigma}$ are formed levelwise, the fact that the Thom space functor preserves these 
constructions $[23, \mathrm{IX}]$ implies that the symmetric Thom spectrum functor has the same property. Given maps of $\mathcal{I}$-spaces $\alpha: A \rightarrow B F$ and $\beta: B \rightarrow B F$, we may view the canonical maps

$$
A(m) \times B(n) \longrightarrow A \otimes B(m+n)
$$

as maps over $B F(m+n)$ and since the Thom space functor takes cartesian products to smash products, the induced maps of Thom spaces take the form

$$
T(\alpha)(m) \wedge T(\beta)(n) \longrightarrow T(\alpha \otimes \beta)(m+n) .
$$

It follows from $[42,1.1]$ that the induced map of symmetric spectra

$$
T(\alpha) \wedge T(\beta) \longrightarrow T(\alpha \otimes \beta)
$$

is an isomorphism such that $T$ defines a strong symmetric monoidal functor. We next discuss homotopy invariance. Applying the usual Hurewicz fibrant replacement functor levelwise (defined using the standard path space fibration) we get an endofunctor $\Gamma$ on $\mathcal{I U} / B F$ and it follows from $[42,1.4]$ that the composite functor

$$
T \Gamma: \mathcal{I U} / B F \stackrel{\Gamma}{\rightarrow} \mathcal{I U} / B F \stackrel{T}{\rightarrow} \mathrm{Sp}^{\Sigma}
$$

is a homotopy functor: if $(A, \alpha) \rightarrow(B, \beta)$ is a weak equivalence over $B F$ (that is, $A_{h \mathcal{I}} \rightarrow B_{h \mathcal{I}}$ is a weak homotopy equivalence), then $T \Gamma(\alpha) \rightarrow T \Gamma(\beta)$ is a stable model equivalence. We say that an object $(A, \alpha)$ is $T$-good if the canonical map $T(\alpha) \rightarrow T \Gamma(\alpha)$ is a stable model equivalence. It follows from the definition that the symmetric Thom spectrum functor preserves weak equivalences on the full subcategory of $T$-good objects in $\mathcal{I U} / B F$. Since $\mathcal{I U} / B O$ maps into the subcategory of $T$-good objects in $\mathcal{I U} / B F$ by $[42,2.3]$, this in particular implies that $T$ preserves weak equivalences when restricted to $\mathcal{I U} / B O$.

Proposition 8.1 Restricted to the full subcategory of $T$-good objects, the two compositions in the diagram

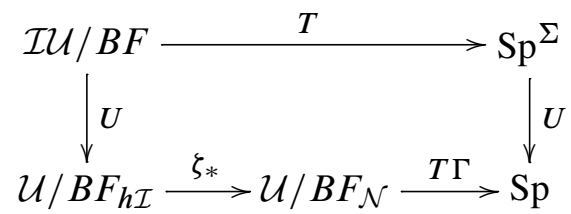

are related by a chain of natural stable equivalences.

Again this implies the statement in (A2) when restricted to $\mathcal{I U} / B O$. We postpone the proof of this result until we have introduced the functor $R$ in (A6). 


\section{Flat replacement and Axiom (A3)}

We first recall the flatness notion for $\mathcal{I}$-spaces introduced in [41]. Thus, an $\mathcal{I}$-space $A$ is flat if for any diagram of the form (7-1), such that the intersection of the images of $\beta_{1}$ and $\beta_{2}$ equals the image of $\gamma$, the induced map

$$
A\left(n_{1}\right) \cup_{A(m)} A\left(n_{2}\right) \longrightarrow A(n)
$$

is an $h$-cofibration. By Lillig's union theorem for $h$-cofibrations [24], this is equivalent to the requirement that (i) any morphism $\alpha: \mathbf{m} \rightarrow \mathbf{n}$ in $\mathcal{I}$ induces an $h$-cofibration $A(m) \rightarrow A(n)$, and (ii) that the intersection of the images of $A\left(n_{1}\right)$ and $A\left(n_{2}\right)$ in $A(n)$ equals the image of $A(m)$. For instance, the $\mathcal{I}$-spaces $B O$ and $B F$ are flat.

The flat replacement of an $\mathcal{I}$-space $A$ is the $\mathcal{I}$-space $\bar{A}$ defined by

$$
\bar{A}(n)=\underset{\alpha: \mathbf{n}_{1} \rightarrow \mathbf{n}}{\operatorname{hocolim}} A\left(n_{1}\right),
$$

where the homotopy colimit is over the category $\mathcal{I} / \mathbf{n}$. We have the following $\mathcal{I}$-space analogues of Propositions 7.7 and 7.9.

Proposition 8.2 The $\mathcal{I}$-space $\bar{A}$ is flat and the canonical projection $\bar{A} \rightarrow A$ is a levelwise equivalence.

Proposition 8.3 The flat replacement functor is a (lax) monoidal functor on IU and $\bar{A} \rightarrow A$ is a monoidal natural transformation.

It follows from this that the flat replacement functor induces a functor on the category of $\mathcal{I}$-space monoids. As in the axiomatic framework from Section 2.2 we say that an $\mathcal{I}$-space monoid is well-based if the unit $* \rightarrow A$ is an $h$-cofibration. Let $I$ be the unit interval, thought of as a based topological monoid with base point 0 and unit 1 . Given a $\mathcal{I}$-space monoid $A$, let $A^{\prime}=A \vee I$ be the $\mathcal{I}$-space monoid defined by the levelwise wedge products $A^{\prime}(n)=A(n) \vee I$. Notice that if $A$ is commutative, then so is $A^{\prime}$. This construction is the $\mathcal{I}$-space analogue of the usual procedure for replacing a topological monoid by one that is well-based. Arguing as in the case of a topological monoid [32, A.8], one deduces the following.

Proposition 8.4 Let $A$ be an $\mathcal{I}$-space monoid. The associated $\mathcal{I}$-space monoid $A^{\prime}=A \vee I$ is well-based and the canonical map of monoids $A^{\prime} \rightarrow A$ is a homotopy equivalence. 
We now define the functor $C$ in (A3) by

$$
C: \mathcal{I U}[\mathbb{T}] \longrightarrow \mathcal{I U}[\mathbb{T}], \quad A \mapsto A^{c}=(\bar{A})^{\prime}
$$

and we define the natural transformation $A^{c} \rightarrow A$ to be the composition of the levelwise weak homotopy equivalences $(\bar{A})^{\prime} \rightarrow \bar{A} \rightarrow A$. Given a monoid morphism $\alpha: A \rightarrow B F$, we write $\alpha^{c}$ for the composition

$$
\alpha^{c}: A^{c} \rightarrow A \stackrel{\alpha}{\rightarrow} B F .
$$

We need a technical assumption to ensure that the associated symmetric spectrum $T\left(\alpha^{c}\right)$ is levelwise well-based. In general, we say that a map of $\mathcal{I}$-spaces $\alpha: A \rightarrow B F$ classifies a well-based $\mathcal{I}$-space over $A$ if the $h$-cofibrations $B F(n) \rightarrow E F(n)$ pull back to $h$-cofibrations via $\alpha$. This condition is automatically satisfied if $\alpha$ factors through $B O$ [23, IX; 42, 2.3]. Thus, restricted to such morphisms the following proposition verifies (A3) in the $B O$ case. Recall the functor $T \mapsto T^{c}$ from Proposition 7.10.

Proposition 8.5 There is an isomorphism of symmetric ring spectra

$$
T\left(\alpha^{c}\right) \cong T(\alpha)^{c}
$$

and if $\alpha$ classifies a well-based $\mathcal{I}$-space over $A$, then $T\left(\alpha^{c}\right)$ is a flat symmetric ring spectrum.

Proof Consider in general a monoid morphism $\alpha: A \rightarrow B F$ and the induced morphisms $\alpha^{\prime}: A^{\prime} \rightarrow B F$ and $\bar{\alpha}: \bar{A} \rightarrow B F$. We claim that there are isomorphisms of symmetric ring spectra $T\left(\alpha^{\prime}\right) \cong T(\alpha)^{\prime}$ and $T(\bar{\alpha}) \cong \overline{T(\alpha)}$. This clearly gives the isomorphism in the proposition. The isomorphism for $\alpha^{\prime}$ follows directly from the fact that $T$ preserves colimits. For the second isomorphism we first observe that since the Thom space functor preserves coproducts and topological realization, it also preserves homotopy colimits. Thus, we have levelwise homeomorphisms

$$
T(\bar{\alpha})(n) \cong \operatorname{hocolim}_{\gamma: \mathbf{n}_{1} \rightarrow \mathbf{n}} T\left(A\left(n_{1}\right) \stackrel{\gamma \circ \alpha_{n_{1}}}{\longrightarrow} B F(n)\right) \cong \underset{\gamma: \mathbf{n}_{1} \rightarrow \mathbf{n}}{\operatorname{hocolim}} S^{n-\gamma} \wedge T(\alpha)\left(n_{1}\right)
$$

and since the last term is $\overline{T(\alpha)}(n)$ by definition, the claim follows. For the last statement in the proposition we observe that if $\alpha$ classifies a well-based $\mathcal{I}$-space over $A$, then the symmetric spectrum $T(\alpha)$ is levelwise well-based. The statement therefore follows from Proposition 7.10.

Remark It is proved in [38] that there is a model structure on $\mathcal{I U}$ whose weak equivalences are the maps that induce weak homotopy equivalences on homotopy colimits. Similarly, there are model structures on the categories of monoids and 
commutative monoids in $\mathcal{I U}$. However, there are several reasons why these model structures are not directly suited for the analysis of Thom spectra. For example, it is not clear that the $T$-goodness condition on objects in $\mathcal{I U} / B F$ is preserved under cofibrant replacement and the Thom spectra associated to cofibrant $\mathcal{I}$-spaces will not in general be cofibrant as symmetric spectra. Using the less restrictive notion of flatness introduced here also makes it clear that many $\mathcal{I}$-spaces, such as for example $B F$, behave well with respect to the $\otimes$-product even though they are not cofibrant in these model structures.

We next formulate some further properties of the flat replacement functor that will be needed later. Consider the homotopy invariant version of the $\otimes$-product defined by

$$
A_{1} \nabla^{h} \cdots \nabla^{h} A_{k}(n)=\underset{\mathbf{n}_{1} \sqcup \cdots \sqcup \mathbf{n}_{k} \rightarrow \mathbf{n}}{\operatorname{hocolim}} A\left(n_{1}\right) \times \cdots \times A\left(n_{k}\right) .
$$

For $k=1$ this is the flat replacement $\bar{A}$. As for the analogous functor for symmetric spectra it should be remarked that the functor on $k$-tuples of $\mathcal{I}$-spaces so defined is not the same as the iteration of the functor $(-) \nabla^{h}(-)$.

Proposition 8.6 There is a natural isomorphism of $\mathcal{I}$-spaces

$$
\bar{A}_{1} \otimes \cdots \otimes \bar{A}_{k} \cong A_{1} \nabla^{h} \cdots \otimes^{h} A_{k} .
$$

We also have the following $\mathcal{I}$-space analogue of Proposition 7.2. The proof is similar to but slightly easier than the symmetric spectrum version since we do not have to worry about base points here.

Proposition $8.7[41,2.5]$ If the $\mathcal{I}$-spaces $A_{1}, \ldots, A_{k}$ are flat, then the canonical projection

$$
A_{1} \otimes^{h} \cdots \otimes^{h} A_{k} \longrightarrow A_{1} \otimes \cdots \otimes A_{k}
$$

is a levelwise equivalence.

As in Section 7.2 we conclude from this that the $\nabla^{h}$-product always represents the "derived" homotopy type and that the $\otimes$-product has the "derived" homotopy type for flat $\mathcal{I}$-spaces.

\section{Axiom (A4)}

We define $Q$ to be the colimit over $\mathcal{I}$,

$$
Q: \mathcal{I U} \longrightarrow \mathcal{U}, \quad Q A=\underset{\mathcal{I}}{\operatorname{colim}} A
$$


It is clear that $Q$ preserves colimits and the fact that $\mathcal{U}$ is closed symmetric monoidal under the categorical product implies that it also preserves tensors. Before verifying the remaining conditions it is helpful to recall some general facts about Kan extensions. Thus, consider in general a functor $\phi: \mathcal{B} \rightarrow \mathcal{C}$ between small categories. Given a $\mathcal{B}$-diagram $X: \mathcal{B} \rightarrow \mathcal{U}$, the (left) Kan extension is the functor $\phi_{*} X: \mathcal{C} \rightarrow \mathcal{U}$ defined by

$$
\phi_{*} X(c)=\underset{\phi / c}{\operatorname{colim}} X \circ \pi_{c}
$$

and the homotopy Kan extension is the functor $\phi_{*}^{h} X: \mathcal{C} \rightarrow \mathcal{U}$ defined by the analogous homotopy colimits,

$$
\phi_{*}^{h} X(c)=\underset{\phi / c}{\operatorname{hocolim}} X \circ \pi_{c} .
$$

Here $\pi_{c}$ denotes the forgetful functor $\phi / c \rightarrow \mathcal{B}$; see eg Schlichtkrull [41]. The effect of evaluating the colimits of these functors is recorded in the following lemma.

Lemma 8.8 There are natural isomorphisms

$$
\underset{\mathcal{C}}{\operatorname{colim}} \phi_{*} X \cong \underset{\mathcal{B}}{\operatorname{colim}} X, \quad \operatorname{colim}_{\mathcal{C}} \phi_{*}^{h} X \cong \underset{\mathcal{B}}{\operatorname{hocolim}} X,
$$

and the canonical projection from the homotopy colimit to the colimit

$$
\underset{\mathcal{C}}{\operatorname{hocolim}} \phi_{*}^{h} X \longrightarrow \underset{\mathcal{C}}{\operatorname{colim}} \phi_{*}^{h} X
$$

is a weak homotopy equivalence.

An explicit proof of the last statement can be found in $[41,1.4]$. Using that the $\nabla-$ product is defined as a Kan extension, the fact that $Q$ is strong symmetric monoidal now follows from the canonical homeomorphisms

$$
\operatorname{colim}_{\mathcal{I}} A \otimes B \cong \operatorname{colim}_{\mathcal{I} \times \mathcal{I}} A \times B \cong \operatorname{colim}_{\mathcal{I}} A \times \operatorname{colim}_{\mathcal{I}} B,
$$

where the second homeomorphism again is deduced from the fact that $\mathcal{U}$ is closed. We define the natural transformation $U \rightarrow Q$ to be the canonical projection from the homotopy colimit to the colimit.

Lemma 8.9 Given $\mathcal{I}$-spaces $A_{1}, \ldots, A_{k}$, there is a canonical homeomorphism

$$
Q\left(\bar{A}_{1} \otimes \cdots \otimes \bar{A}_{k}\right) \cong U A_{1} \times \cdots \times U A_{k}
$$

and the canonical projection gives a weak homotopy equivalence

$$
U\left(\bar{A}_{1} \otimes \cdots \otimes \bar{A}_{k}\right) \stackrel{\simeq}{\rightarrow} Q\left(\bar{A}_{1} \otimes \cdots \otimes \bar{A}_{k}\right) .
$$


Proof Using Proposition 8.6 we may write $\bar{A}_{1} \otimes \cdots \otimes \bar{A}_{k}$ as a homotopy Kan extension, hence the result follows immediately from Lemma 8.8.

When $A$ is an $\mathcal{I}$-space monoid the canonical map $(\bar{A})^{\prime} \rightarrow \bar{A}$ is a homotopy equivalence of $\mathcal{I}$-spaces. The above lemma therefore implies the following result which concludes the verification of the conditions in (A4).

Proposition 8.10 Given $\mathcal{I}$-space monoids $A_{1}, \ldots, A_{k}$, the canonical projection

$$
U\left(A_{1}^{c} \otimes \cdots \otimes A_{k}^{c}\right) \longrightarrow Q\left(A_{1}^{c} \otimes \cdots \otimes A_{k}^{c}\right)
$$

is a weak homotopy equivalence.

\section{Axiom (A5)}

In the notation of Axiom (A5), we define $B F^{\prime}$ and $B O^{\prime}$ to be the well-based $\mathcal{I}$-space monoids defined from $B F$ and $B O$ as in Proposition 8.4. These are again commutative flat $\mathcal{I}$-space monoids and the condition in (A5) therefore follows from the following more general result.

Proposition 8.11 If $A_{1}, \ldots, A_{k}$ are flat $\mathcal{I}$-spaces, then the canonical map

$$
U\left(A_{1} \otimes \cdots \otimes A_{k}\right) \longrightarrow U A_{1} \times \cdots \times U A_{k}
$$

is a weak homotopy equivalence.

Proof Consider the commutative diagram

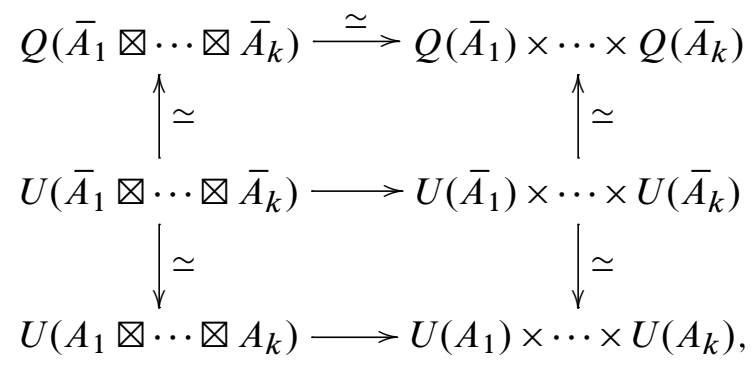

where the vertical maps are weak homotopy equivalences by Proposition 8.7 and Lemma 8.9. The horizontal map on the top is a homeomorphism since $Q$ is strong monoidal and the result follows. 


\section{Axiom (A6)}

The definition of the functor in (A6) is based on the $\mathcal{I}$-space lifting functor

$$
R: \mathcal{U} / B F_{h \mathcal{I}} \longrightarrow \mathcal{I U} / B F, \quad\left(X \stackrel{f}{\rightarrow} B F_{h \mathcal{I}}\right) \mapsto\left(R_{f}(X) \stackrel{R(f)}{\longrightarrow} B F\right)
$$

introduced in [42]. We shall not repeat the details of the definition here, but we remark that a similar construction applies to give an $\mathcal{I}$-space lifting functor with $B O$ instead of $B F$. The following is proved in [42].

Proposition 8.12 [42, 6.8] The Barratt-Eccles operad $\mathcal{E}$ acts on $B F_{h \mathcal{I}}$ and if $\mathcal{C}$ is an operad that is augmented over $\mathcal{E}$, then there is an induced functor

$$
R: \mathcal{U}[\mathcal{C}] / B F_{h \mathcal{I}} \longrightarrow \mathcal{I U}[\mathcal{C}] / B F
$$

and a natural weak equivalence of $\mathcal{C}$-spaces $\left(R_{f}(X)\right)_{h \mathcal{I}} \stackrel{\simeq}{\rightarrow} X$.

There is an analogous result in the $B O$ case. Now let $\mathcal{C}$ be the associativity operad such that the categories $\mathcal{U}[\mathcal{C}]$ and $\mathcal{I U}[\mathcal{C}]$ are the categories of topological monoids and $\mathcal{I}$-space monoids, respectively. The associativity operad is augmented over the Barratt-Eccles operad and we define the functor $R$ in (A6) to be the induced functor

$$
R: \mathcal{U}[\mathcal{C}] / B F_{h \mathcal{I}} \longrightarrow \mathcal{I U}[\mathbb{T}] / B F
$$

and similarly with $B O$ instead of $B F$. The composite functor

$$
\mathcal{U}[\mathcal{C}] / B F_{h \mathcal{I}} \stackrel{R}{\rightarrow} \mathcal{I U}[\mathbb{T}] / B F \longrightarrow \mathcal{I U}[\mathbb{T}] \stackrel{C}{\rightarrow} \mathcal{I U}[\mathbb{T}] \stackrel{Q}{\rightarrow} \mathcal{U}[\mathbb{T}] \longrightarrow \mathcal{U}[\mathcal{C}]
$$

takes an object $f: X \rightarrow B F_{h \mathcal{I}}$ to $Q\left(R_{f}(X)\right)^{c}$ and we have a chain of weak homotopy equivalences in $\mathcal{U}[\mathcal{C}]$ given by

$$
Q\left(R_{f}(X)\right)^{c} \stackrel{\simeq}{\rightarrow} Q\left(\overline{R_{f}(X)}\right) \stackrel{\simeq}{\rightarrow}\left(R_{f}(X)\right)_{h \mathcal{I}} \stackrel{\simeq}{\rightarrow} X .
$$

Here the left hand equivalence is induced by the canonical homotopy equivalence of Proposition 8.4, the next is the homeomorphism established in Lemma 8.9, and the last equivalence is provided by the preceding Proposition. The verification of the axioms is now complete except for the proof of Proposition 8.1. For this we need the following two results from [42]. Recall that by our conventions a map in $\mathcal{I U}$ is a weak equivalence if the induced map of homotopy colimits is a weak homotopy equivalence.

Proposition 8.13 [42, 4.11] The composite functor

$$
\mathcal{I U} / B F \stackrel{U}{\rightarrow} \mathcal{U} / B F_{h \mathcal{I}} \stackrel{R}{\rightarrow} \mathcal{I U} / B F
$$

is related to the identity functor on $\mathcal{I U} / B F$ by a chain of natural weak equivalences. 
The next proposition shows that the ordinary Thom spectrum functor can be recovered from the symmetric Thom spectrum functor up to stable equivalence. It is a direct consequence of $[42,4.10 ; 42,4.23]$.

Proposition 8.14 [42] The two compositions in the diagram

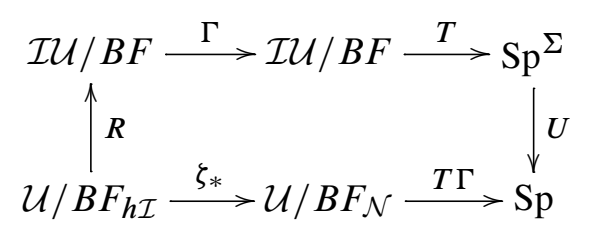

are related by a chain of natural stable equivalences.

Since by $[42,4.9]$ the functor $R$ takes values in the subcategory of $T$-good objects in $\mathcal{I U} / B F$, the composite functor in the proposition is in fact stably equivalent to $U T R$. However, the above formulation is convenient for the application in the following proof.

Proof of Proposition 8.1 It suffices to show that the two compositions in the diagram

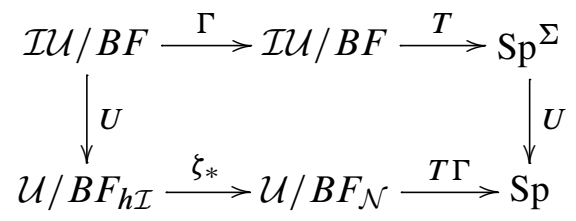

are related by a chain of stable equivalences. Composing the chain of equivalences in Proposition 8.13 with the levelwise fibrant replacement functor $\Gamma$ gives a chain of natural equivalences relating the functors $\Gamma R U$ and $\Gamma$ on $\mathcal{I U} / B F$. Therefore, applying the symmetric Thom spectrum functor to this chain, we get a chain of stable model equivalences

$$
T \Gamma R U \simeq T \Gamma: \mathcal{I U} / B F \longrightarrow \mathrm{Sp}^{\Sigma} \text {. }
$$

Combining this with Proposition 8.14, we finally get the required chain of stable equivalences

$$
U T \Gamma \simeq U T \Gamma R U \simeq T \Gamma \zeta_{*} U
$$

\subsection{The proof of Theorem 1 in the general case}

In this section we show that the weaker forms of the Axioms (A2) and (A3) suffice for the proof of Theorem 1. The main technical difficulty is that the symmetric Thom spectrum functor only preserves weak equivalences on the full subcategory of $T$-good objects in $\mathcal{I U} / B F$. In order to maintain homotopical control we must therefore be careful only to apply the Thom spectrum functor to $T$-good objects. We say that a map $\alpha_{n}: A(n) \rightarrow B F(n)$ classifies a well-based quasifibration if 
(i) the pullback $\alpha^{*} E F(n) \rightarrow A(n)$ is a quasifibration, and

(ii) the induced section $A(n) \rightarrow \alpha^{*} E F(n)$ is an $h$-cofibration.

An object $\alpha$ in $\mathcal{I U} / B F$ is said to classify well-based quasifibrations if the level maps $\alpha_{n}$ do. This condition implies that $\alpha$ is $T$-good and is sometimes technically convenient as in the following lemma from [42].

Lemma 8.15 [42, A.4] Let $\Lambda$ be a small category and let $f_{\lambda}: X_{\lambda} \rightarrow B F(n)$ be a $\Lambda$-diagram in $\mathcal{U} / B F(n)$ such that each $f_{\lambda}$ classifies a well-based quasifibration. Then the induced map

$$
f: \underset{\Lambda}{\operatorname{hocolim}} X_{\lambda} \longrightarrow B F(n)
$$

also classifies a well-based quasifibration.

Now let $f: X \rightarrow B F_{h \mathcal{I}}$ be a map of topological monoids and let $\alpha: A \rightarrow B F$ be the object in $\mathcal{I U}[\mathbb{T}] / B F$ obtained by applying the functor $R$. The first step is to ensure that the symmetric Thom spectra $T(\alpha)$ and $T\left(\alpha^{c}\right)$ have the correct homotopy types.

Lemma 8.16 The objects $\alpha: A \rightarrow B F$ and $\alpha^{c}: A^{c} \rightarrow B F$ classify well-based quasifibrations.

Proof It follows from the definition of the functor $R$ in [42] that the level maps $\alpha_{n}$ classify well-based quasifibrations and that the same holds for the composite maps

$$
A(m) \stackrel{\alpha_{m}}{\longrightarrow} B F(m) \stackrel{\gamma}{\rightarrow} B F(n)
$$

for each morphism $\gamma: \mathbf{m} \rightarrow \mathbf{n}$ in $\mathcal{I}$. Thus, $\alpha$ classifies well-based quasifibrations and by Lemma 8.15 the same holds for $\bar{\alpha}$. Since $A^{c}$ is the homotopy colimit of the diagram $* \rightarrow \bar{A}$, the result follow.

It now follows from Proposition 8.5 that $T\left(\alpha^{c}\right)$ is a flat symmetric ring spectrum and therefore that the cyclic bar construction $B^{\text {cy }}\left(T\left(\alpha^{c}\right)\right)$ represents the topological Hochschild homology of $T(\alpha)$. It remains to analyze the map $B^{\text {cy }}\left(\alpha^{c}\right)$.

Lemma 8.17 The object $B^{\text {cy }}\left(\alpha^{c}\right): B^{\text {cy }}\left(A^{c}\right) \rightarrow B F$ in $\mathcal{I U} / B F$ is $T$-good.

In preparation for the proof, consider in general a simplicial object $f_{\bullet}: X_{\bullet} \rightarrow B F(n)$ in $\mathcal{U} / B F(n)$ with topological realization $f: X \rightarrow B F(n)$. Evaluating the Thom spaces degree-wise we get a simplicial based space $T\left(f_{\bullet}\right)$ whose realization is isomorphic to $T(f)$ as follows from Lemma 3.1. 
Lemma 8.18 Suppose that $f_{\bullet}: X_{\bullet} \rightarrow B F(n)$ is degree-wise $T$-good and that the simplicial spaces $X_{\bullet}$ and $T\left(f_{\bullet}\right)$ are good. Then the realization $f: X \rightarrow B F(n)$ is also $T$-good.

Proof We must show that the canonical map $X \rightarrow \Gamma_{f}(X)$ induces a weak homotopy equivalence of Thom spaces $T(f) \rightarrow T \Gamma(f)$. Using that $\Gamma$ preserves tensors and colimits, we identify the latter map with the realization of the simplicial map $T\left(f_{\bullet}\right) \rightarrow T \Gamma\left(f_{\bullet}\right)$. The assumption that $f$ be degree-wise $T$-good implies that this is a degree-wise weak homotopy equivalence. Since the simplicial space $T\left(f_{\bullet}\right)$ is good by assumption, it remains to show that the goodness assumption on $X_{\bullet}$ implies that $T \Gamma\left(f_{\bullet}\right)$ is also good. For this we use [23, IX.1.11], which implies that the degeneracy maps $\Gamma\left(X_{k}\right) \rightarrow \Gamma\left(X_{k+1}\right)$ are fibrewise $h$-cofibrations over $B F(n)$. The induced maps of Thom spaces are therefore also $h$-cofibrations. The conclusion now follows from the fact that a levelwise weak equivalence of good simplicial spaces induces a weak equivalence after topological realization.

Proof of Lemma 8.17 Notice first that $B_{\bullet}^{\mathrm{cy}}(A)$ and $T\left(B_{\bullet}^{\mathrm{cy}}\left(\alpha^{c}\right)\right)$ are good simplicial objects since $A^{c}$ and $T\left(\alpha^{c}\right)$ are well-based. By the lemma just proved it therefore suffices to show that the simplicial map $B_{\bullet}^{\mathrm{cy}}\left(A^{c}\right)(n) \rightarrow B F(n)$ is degree-wise $T$-good for each $n$. In simplicial degree $k$ this is the composition

$$
A^{c} \otimes \cdots \otimes A^{c}(n) \longrightarrow \bar{A} \otimes \cdots \otimes \bar{A}(n) \longrightarrow B F(n)
$$

where the first map is a fibrewise homotopy equivalence over $B F(n)$. It therefore suffices to show that the second map is $T$-good and using Proposition 8.6 we write the latter as a map of homotopy colimits

$$
\underset{\mathbf{n}_{0} \sqcup \cdots \sqcup \mathbf{n}_{k} \rightarrow \mathbf{n}}{\operatorname{hocolim}} A\left(n_{0}\right) \times \cdots \times A\left(n_{k}\right) \longrightarrow B F(n) .
$$

The maps in the underlying diagram classify well-based quasifibrations by construction and the result now follows from Lemma 8.15.

Proof of Theorem 1 Let $\mathcal{C}$ be the associativity operad. As explained in Appendix A we may assume that our loop map is a map of $\mathcal{C}$-spaces $f: X \rightarrow B F_{h \mathcal{I}}$ and we again write $\alpha: A \rightarrow B F$ for the associated monoid morphism. It follows from the above discussion that the topological Hochschild homology of $T(f)$ is represented by $B^{\text {cy }}\left(T\left(\alpha^{c}\right)\right)$ which in turn is isomorphic to the symmetric Thom spectrum $T\left(B^{\text {cy }}\left(\alpha^{c}\right)\right)$. Since $B^{\text {cy }}\left(\alpha^{c}\right)$ is $T$-good, the weaker version of (A2) suffices to give a stable equivalence

$$
U T\left(B^{\mathrm{cy}}\left(\alpha^{c}\right)\right) \simeq T \Gamma \zeta_{*}\left(U B^{\mathrm{cy}}\left(\alpha^{c}\right)\right)
$$


From here the argument proceeds as in Section 3.3 and we get a stable equivalence

$$
U T\left(B^{\mathrm{cy}}\left(\alpha^{c}\right)\right) \simeq T \Gamma\left(L^{\eta}(B f)\right)
$$

which is the content of Theorem 1.

Proceeding as in Section 3.3 one finally deduces the general case of Theorem 2 and Theorem 3 from this result.

\section{Appendix A Loop maps and $A_{\infty}$ maps}

In order to prove our main results we need to pass from loop map data to the more rigid kind of data specified by a map of $A_{\infty}$ spaces. This can be done using the machinery from [32] as we now explain. Let $\mathcal{C}_{1}$ be the little 1-cubes operad. Given an $A_{\infty}$ operad $\mathcal{C}$ we let $\mathcal{D}$ be the fibred product $\mathcal{C} \nabla \mathcal{C}_{1}$ such that there is a diagram of $A_{\infty}$ operads $\mathcal{C} \leftarrow \mathcal{D} \rightarrow \mathcal{C}_{1}$. Let $C, C_{1}$ and $D$ be the associated monads on the category $\mathcal{T}$ of based spaces. With the notation from [32, 13.1], we have for each $\mathcal{C}$-space $X$ a diagram of $\mathcal{D}$-spaces

$$
X \stackrel{\epsilon}{\leftarrow} B(D, D, X) \stackrel{\gamma}{\rightarrow} \Omega B(\Sigma, D, X)
$$

where $\epsilon$ is a homotopy equivalence. We write $B_{1} X$ for the space $B(\Sigma, D, X)$. If $X$ is grouplike, then this is a delooping in the sense that the map $\gamma$ also is a weak equivalence; see May [32], Segal [44] and Thomason [49]. It is known by [49] that the functor $X \mapsto B_{1} X$ is equivalent to the functor obtained by first replacing $X$ by a topological monoid and then applying the usual classifying space construction (this is the functor denoted $B^{\prime} X$ in Section 3.3). Here and in the following we tacitly assume that all base points are nondegenerate. This is not a serious restriction since by [32] any $A_{\infty}$ space may be functorially replaced by one with a nondegenerate base point. The following result shows that we can always rectify loop maps over a grouplike $A_{\infty}$ space to $A_{\infty}$ maps.

Proposition A.1 Let $\mathcal{C}$ be an $A_{\infty}$ operad and let $Z$ be a grouplike $\mathcal{C}$-space. Then there exists a "loop functor"

$$
\bar{\Omega}: \mathcal{T} / B_{1} Z \longrightarrow \mathcal{U}[\mathcal{C}] / Z, \quad\left(Y \stackrel{g}{\rightarrow} B_{1} Z\right) \mapsto\left(\bar{\Omega}_{g}(Y) \stackrel{\bar{\Omega}(g)}{\longrightarrow} Z\right),
$$


and a chain of weak equivalences of $\mathcal{D}$-spaces $\bar{\Omega}_{g}(Y) \simeq \Omega(Y)$ such that the diagram

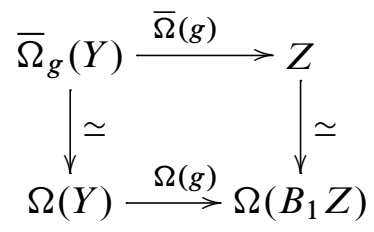

is commutative in the homotopy category.

Proof Given a based map $g: Y \rightarrow B_{1} Z$, let $\Omega_{g}^{\prime}(Y)$ be the homotopy pullback of the diagram of $\mathcal{D}$-spaces

$$
\Omega(Y) \stackrel{\Omega(g)}{\longrightarrow} \Omega\left(B_{1} Z\right) \stackrel{\gamma}{\leftarrow} B(D, D, Z) .
$$

Then $\Omega_{g}^{\prime}(Y)$ is a $\mathcal{D}$-space, and since $Z$ is group-like, the map $\gamma$ is a weak equivalence and therefore the map $\Omega_{g}^{\prime}(Y) \rightarrow \Omega(Y)$ is a weak equivalence of $\mathcal{D}$-spaces. In addition, we have an induced map of $\mathcal{D}$-spaces

$$
\Omega^{\prime}(g): \Omega_{g}^{\prime}(Y) \longrightarrow B(D, D, Z) \stackrel{\epsilon}{\rightarrow} Z .
$$

Consider now the functor $B(C, D,-)$ from $\mathcal{D}$-spaces to $\mathcal{C}$-spaces and notice that there are natural equivalences of $\mathcal{D}$-spaces

$$
X \stackrel{\epsilon}{\leftarrow} B(D, D, X) \longrightarrow B(C, D, X)
$$

for any $\mathcal{D}$-space $X$. We define $\bar{\Omega}(g)$ to be the map of $\mathcal{C}$-spaces induced by $\Omega^{\prime}(g)$,

$$
\bar{\Omega}(g): \bar{\Omega}_{g}(Y)=B\left(C, D, \Omega_{g}^{\prime}(Y)\right) \longrightarrow B(C, D, Z) \longrightarrow Z .
$$

The last map is defined since $Z$ is already a $\mathcal{C}$-space. It is clear from the construction that $\bar{\Omega}(g)$ is related to $\Omega(g)$ by a homotopy commutative diagram as stated.

Proposition A.2 Let $\bar{\Omega}$ be the functor from Proposition A.1 and suppose that $Z$ is connected. Restricted to $g: Y \rightarrow B_{1} Z$ with $Y$ connected, there is a chain of weak equivalences $Y \simeq B_{1} \bar{\Omega}_{g}(Y)$ such that the diagram

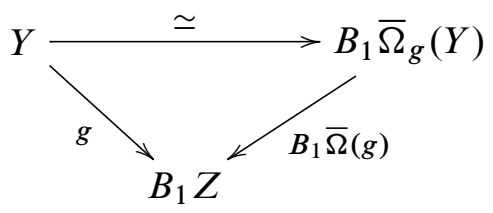

is commutative in the homotopy category. 
Proof Given a based space $Y$ there is a canonical map $\rho: B_{1} \Omega(Y) \rightarrow Y$ which by $[32,13.1]$ and [49] is a weak equivalence if $Y$ is connected. Applying the functor $B_{1}$ to the diagram of $\mathcal{D}$-spaces in Proposition A.1 we therefore get a homotopy commutative diagram

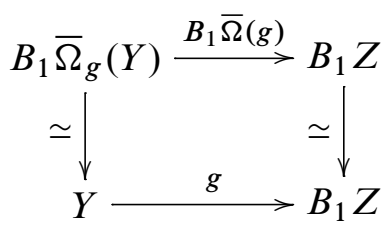

where the vertical arrow on the right represents the chain of maps

$$
B_{1} Z \stackrel{B(\Sigma, D, \epsilon)}{\longleftarrow} B(\Sigma, D, B(D, D, Z)) \stackrel{B(\Sigma, D, \gamma)}{\longrightarrow} B\left(\Sigma, D, \Omega\left(B_{1} Z\right)\right) \stackrel{\rho}{\rightarrow} B_{1} Z .
$$

It remain to show that this represents the identity on $B_{1} Z$. For this we observe that $B(\Sigma, D, B(D, D, Z))$ is homeomorphic to the realization of the bisimplicial space with $(p, q)$-simplices $\Sigma D^{p} D D^{q} Z$ and that the two maps correspond respectively to multiplication in the $p$ and $q$ direction. We may also view this space as the realization of the diagonal simplicial space $\Sigma D^{p} D D^{p} Z$ and the result now follows from the explicit homotopy

$$
H: B(\Sigma, D, B(D, D, Z)) \times I \longrightarrow B_{1} Z, \quad H([b, u], t)=[b, t u,(1-t) u] .
$$

Here $I$ denotes the unit interval, $b$ is an element in $\Sigma D^{p} D D^{p} Z$, and $u$ is an element in the standard $p$-simplex

$$
\Delta^{p}=\left\{\left(u_{0}, \ldots, u_{p}\right) \in I^{p+1}: u_{0}+\cdots+u_{p}=1\right\} .
$$

For completeness we finally show that one may rectify $n$-fold loop maps over a grouplike $E_{\infty}$ space to maps of $E_{n}$-spaces for all $n$. Let $\mathcal{C}_{n}$ be the little $n$-cubes operad. Given an $E_{\infty}$ operad $\mathcal{C}$ we let $\mathcal{D}_{n}$ be the product operad $\mathcal{C} \times \mathcal{C}_{n}$. This is an $E_{n}$ operad in the sense that the projection $\mathcal{D}_{n} \rightarrow \mathcal{C}_{n}$ is an equivalence. We again write $C, C_{n}$ and $D_{n}$ for the associated monads. With the notation from [32, 13.1], we have a diagram of $D_{n}$-maps

$$
X \stackrel{\epsilon}{\leftarrow} B\left(D_{n}, D_{n}, X\right) \stackrel{\gamma}{\rightarrow} \Omega^{n} B\left(\Sigma^{n}, D_{n}, X\right)
$$

and we write $B_{n} X$ for the space $B\left(\Sigma^{n}, D_{n}, X\right)$. When $X$ is grouplike this is an $n$-fold delooping in the sense that $\gamma$ is a weak equivalences. An argument similar to that used in the proof of Proposition A.1 then gives the following. 
Proposition A.3 Let $\mathcal{C}$ be an $E_{\infty}$ operad and let $Z$ be a grouplike $\mathcal{C}$-space. Then there exists an " $n$-fold loop functor"

$$
\bar{\Omega}^{n}: \mathcal{T} / B_{n} Z \longrightarrow \mathcal{U}\left[\mathcal{D}_{n}\right] / Z, \quad\left(Y \stackrel{g}{\rightarrow} B_{n} Z\right) \mapsto\left(\bar{\Omega}_{g}^{n}(Y) \stackrel{\bar{\Omega}^{n}(g)}{\longrightarrow} Z\right),
$$

and a chain of weak equivalences of $\mathcal{D}_{n}$-spaces $\bar{\Omega}_{g}^{n}(Y) \simeq \Omega^{n}(Y)$ such that the diagram

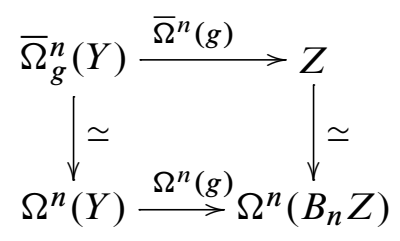

is commutative in the homotopy category.

\section{References}

[1] M Ando, A J Blumberg, D Gepner, M J Hopkins, C Rezk, Units of ring spectra and Thom spectra arXiv:0810.4535v3

[2] A Baker, B Richter, Quasisymmetric functions from a topological point of view, Math. Scand. 103 (2008) 208-242 MR2484353

[3] M Basterra, André-Quillen cohomology of commutative S-algebras, J. Pure Appl. Algebra 144 (1999) 111-143 MR1732625

[4] M Basterra, MA Mandell, Homology and cohomology of $E_{\infty}$ ring spectra, Math. Z. 249 (2005) 903-944 MR2126222

[5] A J Blumberg, Topological Hochschild homology of Thom spectra which are $E_{\infty}$ ring spectra arXiv:0811.0803

[6] A J Blumberg, Progress towards the calculation of the $K$-theory of Thom spectra, $\mathrm{PhD}$ thesis, University of Chicago (2005)

[7] J M Boardman, R M Vogt, Homotopy-everything H-spaces, Bull. Amer. Math. Soc. 74 (1968) 1117-1122 MR0236922

[8] M Bökstedt, The topological Hochschild homology of $\mathbb{Z}$ and $\mathbb{Z} / p$, Preprint (1985)

[9] A K Bousfield, E M Friedlander, Homotopy theory of $\Gamma$-spaces, spectra, and bisimplicial sets, from: "Geometric applications of homotopy theory (Proc. Conf., Evanston, Ill., 1977), II”, Lecture Notes in Math. 658, Springer, Berlin (1978) 80-130 MR513569

[10] A K Bousfield, D M Kan, Homotopy limits, completions and localizations, Lecture Notes in Math. 304, Springer, Berlin (1972) MR0365573

[11] M Brun, $\mathbf{Z}$ Fiedorowicz, R M Vogt, On the multiplicative structure of topological Hochschild homology, Algebr. Geom. Topol. 7 (2007) 1633-1650 MR2366174 
[12] S Bullett, Permutations and braids in cobordism theory, Proc. London Math. Soc. (3) 38 (1979) 517-531 MR532985

[13] F R Cohen, Braid orientations and bundles with flat connections, Invent. Math. 46 (1978) 99-110 MR493954

[14] F R Cohen, J P May, L R Taylor, $K(\mathbf{Z}, 0)$ and $K\left(Z_{2}, 0\right)$ as Thom spectra, Illinois J. Math. 25 (1981) 99-106 MR602900

[15] W G Dwyer, J Spaliński, Homotopy theories and model categories, from: "Handbook of algebraic topology”, (I M James, editor), North-Holland, Amsterdam (1995) 73-126 MR1361887

[16] A D Elmendorf, I Kriz, MA Mandell, J P May, Rings, modules, and algebras in stable homotopy theory, Math. Surveys and Monogr. 47, Amer. Math. Soc. (1997) MR1417719 With an appendix by M Cole

[17] A D Elmendorf, I Kriz, MA Mandell, JP May, Errata for "Rings, modules, and algebras in stable homotopy theory" (2007) Available at http:// www . math . uchicago. edu/ may/BOOKS/Addenda.pdf

[18] T G Goodwillie, Cyclic homology, derivations, and the free loopspace, Topology 24 (1985) 187-215 MR793184

[19] PS Hirschhorn, Model categories and their localizations, Math. Surveys and Monogr. 99, Amer. Math. Soc. (2003) MR1944041

[20] M Hovey, B Shipley, J Smith, Symmetric spectra, J. Amer. Math. Soc. 13 (2000) 149-208 MR1695653

[21] N Iwase, A continuous localization and completion, Trans. Amer. Math. Soc. 320 (1990) 77-90 MR1031978

[22] I Kriz, J P May, Operads, algebras, modules and motives, Astérisque 233 (1995) MR1361938

[23] L G Lewis, Jr, J P May, M Steinberger, J E McClure, Equivariant stable homotopy theory, Lecture Notes in Math. 1213, Springer, Berlin (1986) MR866482 With contributions by $\mathrm{J} \mathrm{E}$ McClure

[24] J Lillig, A union theorem for cofibrations, Arch. Math. (Basel) 24 (1973) 410-415 MR0334193

[25] S Mac Lane, Categories for the working mathematician, second edition, Graduate Texts in Math. 5, Springer, New York (1998) MR1712872

[26] I Madsen, Algebraic $K$-theory and traces, from: "Current developments in mathematics, 1995 (Cambridge, MA)”, (R Bott, M Hopkins, A Jaffe, I Singer, D Stroock, S-T Yau, editors), Int. Press, Cambridge, MA (1994) 191-321 MR1474979

[27] M Mahowald, Ring spectra which are Thom complexes, Duke Math. J. 46 (1979) 549-559 MR544245 
[28] M Mahowald, D C Ravenel, P Shick, The Thomified Eilenberg-Moore spectral sequence, from: "Cohomological methods in homotopy theory (Bellaterra, 1998)", (J Aguadé, C Broto, C Casacuberta, editors), Progr. Math. 196, Birkhäuser, Basel (2001) 249-262 MR1851257

[29] M Mahowald, N Ray, A note on the Thom isomorphism, Proc. Amer. Math. Soc. 82 (1981) 307-308 MR609673

[30] MA Mandell, Topological Hochschild homology of an $E_{n}$ ring spectrum is $E_{n-1}$, Preprint (2004)

[31] M A Mandell, J P May, S Schwede, B Shipley, Model categories of diagram spectra, Proc. London Math. Soc. (3) 82 (2001) 441-512 MR1806878

[32] J P May, The geometry of iterated loop spaces, Lectures Notes in Math. 271, Springer, Berlin (1972) MR0420610

[33] J P May, Classifying spaces and fibrations, Mem. Amer. Math. Soc. 155 (1975) MR0370579

[34] J P May, $E_{\infty}$ ring spaces and $E_{\infty}$ ring spectra, Lecture Notes in Math. 577, Springer, Berlin (1977) MR0494077 With contributions by F Quinn, N Ray, and J Tornehave

[35] J P May, Fibrewise localization and completion, Trans. Amer. Math. Soc. 258 (1980) 127-146 MR554323

[36] J P May, J Sigurdsson, Parametrized homotopy theory, Math. Surveys and Monogr. 132, Amer. Math. Soc. (2006) MR2271789

[37] J R Munkres, Topology: a first course, second edition, Prentice-Hall Inc., Englewood Cliffs, NJ (2000) MR0464128

[38] S Sagave, C Schlichtkrull, Diagram spaces and symmetric spectra, in preparation

[39] C Schlichtkrull, Higher topological Hochschild homology of Thom spectra arXiv: 0811.0597

[40] C Schlichtkrull, Units of ring spectra and their traces in algebraic $K$-theory, Geom. Topol. 8 (2004) 645-673 MR2057776

[41] C Schlichtkrull, The homotopy infinite symmetric product represents stable homotopy, Algebr. Geom. Topol. 7 (2007) 1963-1977 MR2366183

[42] C Schlichtkrull, Thom spectra that are symmetric spectra, Doc. Math. 14 (2009) 699-748 MR2578805

[43] S Schwede, B E Shipley, Algebras and modules in monoidal model categories, Proc. London Math. Soc. (3) 80 (2000) 491-511 MR1734325

[44] G Segal, Configuration-spaces and iterated loop-spaces, Invent. Math. 21 (1973) 213221 MR0331377

[45] G Segal, Categories and cohomology theories, Topology 13 (1974) 293-312 MR0353298 
[46] B Shipley, Symmetric spectra and topological Hochschild homology, K-Theory 19 (2000) 155-183 MR1740756

[47] R E Stong, Notes on cobordism theory, Math. notes, Princeton Univ. Press (1968) MR0248858

[48] A Strøm, The homotopy category is a homotopy category, Arch. Math. (Basel) 23 (1972) 435-441 MR0321082

[49] R W Thomason, Uniqueness of delooping machines, Duke Math. J. 46 (1979) 217-252 MR534053

[50] F Waldhausen, Algebraic K-theory of topological spaces. II, from: "Algebraic topology, Aarhus 1978 (Proc. Sympos., Univ. Aarhus, 1978)”, (J L Dupont, I Madsen, editors), Lecture Notes in Math. 763, Springer, Berlin (1979) 356-394 MR561230

[51] G W Whitehead, Elements of homotopy theory, Graduate Texts in Math. 61, Springer, New York (1978) MR516508

AJB, RLC: Department of Mathematics, Stanford University

Stanford, CA 94305, United States

CS: Department of Mathematics, University of Bergen

Johannes Brunsgate 12, 5008 Bergen, Norway

blumberg@math.stanford.edu, ralph@math.stanford.edu, krull@math.uib.no

Proposed: Paul Goerss

Seconded: Bill Dwyer, Haynes Miller
Received: 4 November 2008

Revised: 1 March 2010 